NBER WORKING PAPER SERIES

\title{
MIS-CLASSIFIED, BINARY, ENDOGENOUS REGRESSORS: IDENTIFICATION AND INFERENCE
}

\author{
Francis J. DiTraglia \\ Camilo García-Jimeno \\ Working Paper 23814 \\ http://www.nber.org/papers/w23814
NATIONAL BUREAU OF ECONOMIC RESEARCH
1050 Massachusetts Avenue
Cambridge, MA 02138
September 2017

We thank Daron Acemoglu, Manuel Arellano, Kristy Buzard, Xu Cheng, Bernardo da Silveira, Bo Honoré, Arthur Lewbel, Chuck Manski, Sophocles Mavroeidis, Francesca Molinari, Yuya Takahashi, and seminar participants at Cambridge, CEMFI, Chicago Booth, Manchester, Northwestern, Oxford, Penn State, Princeton, UCL, the 2016 Greater New York Area Econometrics Colloquium, Camp Econometrics IX, and the 2017 North American Summer Meeting of the Econometric Society for valuable comments and suggestions. This document supersedes an earlier version entitled "On Mis-measured Binary Regressors: New Results and Some Comments on the Literature." The views expressed herein are those of the authors and do not necessarily reflect the views of the National Bureau of Economic Research.

NBER working papers are circulated for discussion and comment purposes. They have not been peer-reviewed or been subject to the review by the NBER Board of Directors that accompanies official NBER publications.

(C) 2017 by Francis J. DiTraglia and Camilo García-Jimeno. All rights reserved. Short sections of text, not to exceed two paragraphs, may be quoted without explicit permission provided that full credit, including $\odot$ notice, is given to the source. 
Mis-classified, Binary, Endogenous Regressors: Identification and Inference

Francis J. DiTraglia and Camilo García-Jimeno

NBER Working Paper No. 23814

September 2017

JEL No. C10,C18,C25,C26

\begin{abstract}
$\underline{\text { ABSTRACT }}$
This paper studies identification and inference for the effect of a mis-classified, binary, endogenous regressor when a discrete-valued instrumental variable is available. We begin by showing that the only existing point identification result for this model is incorrect. We go on to derive the sharp identified set under mean independence assumptions for the instrument and measurement error, and that these fail to point identify the effect of interest. This motivates us to consider alternative and slightly stronger assumptions: we show that adding second and third moment independence assumptions suffices to identify the model. We then turn our attention to inference. We show that both our model, and related models from the literature that assume regressor exogeneity, suffer from weak identification when the effect of interest is small. To address this difficulty, we exploit the inequality restrictions that emerge from our derivation of the sharp identified set under mean independence only. These restrictions remain informative irrespective of the strength of identification. Combining these with the moment equalities that emerge from our identification result, we propose a robust inference procedure using tools from the moment inequality literature. Our method performs well in simulations.
\end{abstract}

Francis J. DiTraglia

Department of Economics

University of Pennsylvania

3718 Locust Walk

Philadelphia

Pennsylvania

19104

fditra@sas.upenn.edu

Camilo García-Jimeno

Department of Economics

University of Pennsylvania

3718 Locust Walk

Philadelphia, PA 19104

and NBER

gcamilo@sas.upenn.edu 


\section{Introduction}

Measurement error and endogeneity are pervasive features of economic data. Conveniently, a valid instrumental variable corrects for both problems when the measurement error is classical, i.e. uncorrelated with the true value of the regressor. Many regressors of interest in applied work, however, are binary and thus cannot be subject to classical measurement error. ${ }^{1}$ When faced with non-classical measurement error, the instrumental variables estimator can be severely biased. In this paper, we study an additively separable model of the form

$$
y=c(\mathbf{x})+\beta(\mathbf{x}) T^{*}+\varepsilon
$$

where $\varepsilon$ is a mean-zero error term, $T^{*}$ is a binary, potentially endogenous regressor of interest, and $\mathbf{x}$ is a vector of exogenous controls. ${ }^{2}$ Our question is whether, and if so under what conditions, a discrete instrumental variable $z$ suffices to non-parametrically identify the causal effect $\beta(\mathbf{x})$ of $T^{*}$, when we observe not $T^{*}$ but a mis-classified binary surrogate $T$.

We proceed under the assumption of non-differential measurement error. This condition has been widely used in the existing literature and imposes that $T$ provides no additional information beyond that contained in $\left(T^{*}, \mathbf{x}\right)$. Even in this fairly standard setting, identification remains an open question: we begin by showing that the only existing identification result for this model is incorrect. We then go on to derive the sharp identified set under the standard first-moment assumptions from the related literature. We show that regardless of the number of values that $z$ takes on, the model is not point identified. This motivates us to consider alternative, and slightly stronger assumptions. We show that, given a binary instrument, the addition of a second moment independence assumption suffices to identify a model with one-sided mis-classification. Adding a second moment restriction on the measurement error along with a third moment independence assumption for the instrument suffices to identify the model in general. This result likewise requires only a binary $z$.

We then turn our attention to inference, showing that both our model and related models from the literature suffer from a weak identification problem. In essence, binary misclassification creates a mixture model and to correct the bias in the instrumental variables estimator, we must estimate the mixing probabilities. But when $\beta(\mathbf{x})$ is small the "mixture modes" are nearly indistinguishable, making it impossible to reliably estimate these proba-

\footnotetext{
${ }^{1}$ The only way to mis-classify a true one is downwards, as a zero, while the only way to mis-classify a true zero is upwards, as a one. This creates negative dependence between the true value of the regressor and the error.

${ }^{2}$ Because $T^{*}$ is binary, there is no loss of generality from writing the model in this form rather than the more familiar $y=h\left(T^{*}, \mathbf{x}\right)+\varepsilon$. Simply define $\beta(\mathbf{x})=h(1, \mathbf{x})-h(0, \mathbf{x})$ and $c(\mathbf{x})=h(0, \mathbf{x})$.
} 
bilities. To address this difficulty, we exploit the inequality moment restrictions that emerge from our derivation of the sharp identified set. These restrictions remain informative even when $\beta(\mathbf{x})$ is small or zero. Combining them with the moment equalities that emerge from our identification result, we propose an identification robust procedure for uniformly valid inference using tools from the moment inequality literature. Our procedure is computationally attractive and performs well in simulations. Moreover, it can be used both in our model and related models from the literature that assume an exogenous $T^{*}$.

Our work relates to a large literature studying departures from the textbook linear, classical measurement error setting. One strand of this literature considers relaxing the assumption of linearity while maintaining that of classical measurement error. Schennach (2004), for example, uses repeated measures of each mis-measured regressor to obtain identification, while Schennach (2007) uses an instrumental variable. More recently, Song et al. (2015) rely on a repeated measure of the mis-measured regressor and the existence of a set of additional regressors, conditional upon which the regressor of interest is unrelated to the unobservables, to obtain identification. For comprehensive reviews of the challenges of addressing measurement error in non-linear models, see Chen et al. (2011) and Schennach (2013). Another strand of the literature considers relaxing the assumption of classical measurement error, by allowing the measurement error to be related to the true value of the unobserved regressor. Chen et al. (2005) obtain identification in a general class of moment condition models with mis-measured data by relying on the existence of an auxiliary dataset from which they can estimate the measurement error process. In contrast, Hu and Shennach (2008) and Song (2015) rely on an instrumental variable and an additional conditional location assumption on the measurement error distribution. More recently, Hu et al. (2015) use a continuous instrument to identify the ratio of partial effects of two continuous regressors, one measured with error, in a linear single index model. Unfortunately, these approaches cannot be applied to the case of a mis-measured binary regressor.

A number of papers have studied models with an exogenous binary regressor subject to non-differential measurement error. One group of papers asks what can be learned without recourse to an instrumental variable. An early contribution by Aigner (1973) characterizes the asymptotic bias of OLS in this setting, and proposes a correction using outside information on the mis-classification process. Related work by Bollinger (1996) provides partial identification bounds. More recently, Chen et al. (2008a) use higher moment assumptions to obtain identification in a linear model, and Chen et al. (2008b) extend these results to the non-parametric setting. van Hasselt and Bollinger (2012) and Bollinger and van Hasselt (2015) provide additional partial identification results. For results on the partial identification of discrete probability distributions under mis-classification, see Molinari (2008). 
Continuing under the assumption of exogeneity and non-differential measurement error, another group of papers relies on the availability of either an instrumental variable or a second measure of $T^{*}$. Black et al. (2000) and Kane et al. (1999) consider a linear model and show that when two alternative measures $T_{1}$ and $T_{2}$ of $T^{*}$ are available, a non-linear GMM estimator can be used to recover the effect of interest. Subsequently, Frazis and Loewenstein (2003) note that an instrumental variable can take the place of one of the measures. Mahajan (2006) extends the results of Black et al. (2000) and Kane et al. (1999) to a more general setting using a binary instrument in place of one of the treatment measures, establishing nonparametric identification of the conditional mean function. When $T^{*}$ is in fact exogenous, this coincides with the causal effect. Hu (2008) derives related results when the mis-classified discrete regressor may take on more than two values. Lewbel (2007) provides an identification result for the same model as Mahajan (2006) under different assumptions. In particular, his "instrument-like variable" need not satisfy the usual exclusion restriction so long as it does not interact with $T^{*}$ and takes on three or more values.

Much less is known about the case in which a binary, or discrete, regressor is not only mis-classified but endogenous. The first paper to provide a formal result for this case is Mahajan (2006). He extends his main result to the case of an endogenous treatment, providing an explicit proof of identification under the usual IV assumption in a model with additively separable errors. As we show below, however, this result is false. ${ }^{3}$ Several more recent papers also consider the case of a mis-classified, endogenous, binary regressor. Kreider et al. (2012), partially identify the effects of food stamps on health outcomes of children under weak measurement error assumptions by relying on auxiliary data. Similarly, Battistin et al. (2014) study the returns to schooling in a setting with multiple mis-reported measures of educational qualifications. Unlike these two papers, our approach does not depend on the availability of auxiliary data. In a different vein, Shiu (2015) uses an exclusion restriction for the participation equation and an additional valid instrument to identify the effect of a discrete, mis-classified endogenous regressor in a semi-parametric selection model. Similarly, Nguimkeu et al. (2016) use exclusion restrictions for both the participation equation and measurement error equation to identify a parametric model with endogenous participation and one-sided endogenous mis-reporting. Unlike those of the preceding two papers, our results rely neither on parametric assumptions nor additional exclusion restrictions. Other than Mahajan (2006), the paper most closely related to our own is that of Ura (2015), who derives partial identification results for a local average treatment effect without assuming non-differential measurement error. Unlike Ura (2015) we study an additively separable model under non-differential measurement error and derive both partial and point identifi-

\footnotetext{
${ }^{3}$ Appendix B provides a detailed explanation of the error in Mahajan's proof.
} 
cation results.

Our work also relates to a large literature on inference using inequality moment conditions. In particular, we adopt the generalized moment selection (GMS) approach of Andrews and Soares (2010) to construct a procedure for identification-robust inference that combines the moment equalities from our point identification results with inequalities from our partial identification results. Although the equalities alone globally identify our model, the inequalities turn out to be extremely valuable in settings where $\beta(\mathbf{x})$ may be small. Although our specific approach differs from theirs, the idea of including moment inequalities in a model that is already point identified by a collection of moment equalities relates to work by Moon and Schorfheide (2009). While the weak identification problem that we point out and address here also emerges in several closely related models, e.g. (Mahajan, 2006) and Frazis and Loewenstein (2003), we are unaware of any other work from the literature that acknowledges

or addresses it. As shown in Appendix C, our inference procedure can be applied to the case of an exogenous regressor with only minor modifications.

The remainder of the paper is organized as follows. Section 2.1 describes our model and assumptions, Section 2.2 relates our results to existing work, and Sections 2.3-2.4 present our identification results. Section 3.1 points out the special inferential difficulties that arise in models with mis-classification while Section 3.2 gives a high-level overview of our proposed inference procedure. Full details of the procedure follow in Sections 3.3-3.5. Section 4 presents simulation results, and Section 5 concludes. Proofs appear in Appendix A, and we give a detailed explanation of the error in Mahajan (2006) in Appendix B.

\section{Identification Results}

\section{$2.1 \quad$ Baseline Assumptions}

As defined in the preceding section, our model is $y=c(\mathbf{x})+\beta(\mathbf{x}) T^{*}+\varepsilon$, where $\varepsilon$ is a meanzero error term, and the parameter of interest is $\beta(\mathbf{x})$ - the effect of an unobserved, binary, endogenous regressor $T^{*}$. Suppose we observe a valid and relevant binary instrument $z$. In the discussion following Corollary 2.3 below, we explain how these results generalize to the case of an arbitrary discrete-valued instrument. We assume that the model and instrument satisfy the following conditions:

\section{Assumption 2.1.}

(i) $y=c(\mathbf{x})+\beta(\mathbf{x}) T^{*}+\varepsilon$ where $T^{*} \in\{0,1\}$ and $\mathbb{E}[\varepsilon]=0$;

(ii) $z \in\{0,1\}$, where $0<\mathbb{P}(z=1 \mid \mathbf{x})<1$, and $\mathbb{P}\left(T^{*}=1 \mid \mathbf{x}, z=1\right) \neq \mathbb{P}\left(T^{*}=1 \mid \mathbf{x}, z=0\right)$; 
(iii) $\mathbb{E}[\varepsilon \mid \mathbf{x}, z]=0$.

Assumptions 2.1(ii) and (iii) are the standard instrument relevance and mean independence assumptions. ${ }^{4}$ If $T^{*}$ were observed, Assumption 2.1 would suffice to identify $\beta(\mathbf{x})$. Unfortunately we observe not $T^{*}$ but a mis-classified binary surrogate $T$. Define the following mis-classification probabilities:

$$
\alpha_{0}(\mathbf{x}, z)=\mathbb{P}\left(T=1 \mid T^{*}=0, \mathbf{x}, z\right), \quad \alpha_{1}(\mathbf{x}, z)=\mathbb{P}\left(T=0 \mid T^{*}=1, \mathbf{x}, z\right)
$$

Following the existing literature for the case of an exogenous regressor (Black et al., 2000; Frazis and Loewenstein, 2003; Kane et al., 1999; Lewbel, 2007; Mahajan, 2006), we impose the following conditions on the mis-classification process.

\section{Assumption 2.2.}

(i) $\alpha_{0}(\mathbf{x}, z)=\alpha_{0}(\mathbf{x}), \alpha_{1}(\mathbf{x}, z)=\alpha_{1}(\mathbf{x})$

(ii) $\alpha_{0}(\mathbf{x})+\alpha_{1}(\mathbf{x})<1$

(iii) $\mathbb{E}\left[\varepsilon \mid \mathbf{x}, z, T^{*}, T\right]=\mathbb{E}\left[\varepsilon \mid \mathbf{x}, z, T^{*}\right]$

Assumption 2.2 (i) states that the mis-classification probabilities do not depend on $z$. As we maintain this assumption throughout, we drop the dependence of $\alpha_{0}$ and $\alpha_{1}$ on $z$ and write $\alpha_{0}(\mathbf{x})$ and $\alpha_{1}(\mathbf{x})$. Assumption 2.2 (ii) restricts the extent of mis-classification and is equivalent to requiring that $T$ and $T^{*}$ be positively correlated. Assumption 2.2 (iii) is often referred to as "non-differential measurement error." Intuitively, it maintains that $T$ provides no additional information about $\varepsilon$, and hence $y$, given knowledge of $\left(T^{*}, z, \mathbf{x}\right)$.

\subsection{Point Identification Results from the Literature}

Existing results from the literature - see for example Frazis and Loewenstein (2003) and Mahajan (2006) - establish that $\beta(\mathbf{x})$ is point identified if Assumptions 2.1-2.2 are augmented to include the following condition:

Assumption 2.3 (Joint Exogeneity). $\mathbb{E}\left[\varepsilon \mid \mathbf{x}, z, T^{*}\right]=0$.

Assumption 2.3 strengthens the mean independence condition from Assumption 2.1 (iii) to hold jointly for $T^{*}$ and $z$. By iterated expectations, this implies that $T^{*}$ is exogenous,

\footnotetext{
${ }^{4}$ Assumption 2.1 (ii) states that $z$ is a relevant instrument for the unobserved regressor $T^{*}$. Under Assumption 2.2, however, this is equivalent to assuming that $z$ is a relevant instrument for the observed regressor $T$ by Lemma 2.1 below.
} 
i.e. $\mathbb{E}\left[\varepsilon \mid \mathbf{x}, T^{*}\right]=0$. If $T^{*}$ is endogenous, Assumption 2.3 clearly fails. Mahajan (2006) argues, however, that the following restriction, along with our Assumptions 2.1-2.2, suffices to identify $\beta(\mathbf{x})$ when $T^{*}$ may be endogenous:

Assumption 2.4 (Mahajan (2006) Equation 11). $\mathbb{E}\left[\varepsilon \mid \mathbf{x}, z, T^{*}, T\right]=\mathbb{E}\left[\varepsilon \mid \mathbf{x}, T^{*}\right]$.

Assumption 2.4 does not require $\mathbb{E}\left[\varepsilon \mid \mathbf{x}, T^{*}\right]$ to be zero, but maintains that it does not vary with $z$. We show in Appendix B, however, that under Assumptions 2.1-2.2, Assumption 2.4 can only hold if $T^{*}$ is exogenous. If $z$ is a valid instrument and $T^{*}$ is endogenous, then Assumption 2.4 implies that there is no first-stage relationship between $z$ and $T^{*}$. As such, identification in the case where $T^{*}$ is endogenous is an open question.

\subsection{Partial Identification}

In this section we derive the sharp identified set under Assumptions 2.1-2.2 and show that $\beta(\mathbf{x})$ is not point identified. To simplify the notation, define the following shorthand for the unobserved and observed first stage probabilities

$$
\begin{aligned}
& p_{k}^{*}(\mathbf{x})=\mathbb{P}\left(T^{*}=1 \mid \mathbf{x}, z=k\right) \\
& p_{k}(\mathbf{x})=\mathbb{P}(T=1 \mid \mathbf{x}, z=k) .
\end{aligned}
$$

We first state two lemmas that have appeared in various guises throughout the literature. These will be used repeatedly below.

Lemma 2.1. Under Assumption 2.2 (i),

$$
\begin{aligned}
{\left[1-\alpha_{0}(\mathbf{x})-\alpha_{1}(\mathbf{x})\right] p_{k}^{*}(\mathbf{x}) } & =p_{k}(\mathbf{x})-\alpha_{0}(\mathbf{x}) \\
{\left[1-\alpha_{0}(\mathbf{x})-\alpha_{1}(\mathbf{x})\right]\left[1-p_{k}^{*}(\mathbf{x})\right] } & =1-p_{k}(\mathbf{x})-\alpha_{1}(\mathbf{x})
\end{aligned}
$$

where the first-stage probabilities $p_{k}^{*}(\mathbf{x})$ and $p_{k}(\mathbf{x})$ are as defined in Equations 3-4.

Lemma 2.2. Under Assumptions 2.1 and 2.2 (i)-(ii),

$$
\beta(\mathbf{x}) \operatorname{Cov}(z, T \mid \mathbf{x})=\left[1-\alpha_{0}(\mathbf{x})-\alpha_{1}(\mathbf{x})\right] \operatorname{Cov}(y, z \mid \mathbf{x})
$$

Lemma 2.1 relates the observed first-stage probabilities $p_{k}(\mathbf{x})$ to their unobserved counterparts $p_{k}^{*}(\mathbf{x})$ in terms of the mis-classification probabilities $\alpha_{0}(\mathbf{x})$ and $\alpha_{1}(\mathbf{x})$. By Assumption 2.2 (ii), $1-\alpha_{0}(\mathbf{x})-\alpha_{1}(\mathbf{x})>0$ so that Lemma 2.1 provides non-trivial bounds for $\alpha_{0}(\mathbf{x})$ and $\alpha_{1}(\mathbf{x})$ in terms of the observed first-stage probabilities. Lemma 2.2 relates the instrumental variables (IV) estimand, $\operatorname{Cov}(y, z \mid \mathbf{x}) / \operatorname{Cov}(z, T \mid \mathbf{x})$, to the mis-classification probabilities. 
Since $1-\alpha_{0}(\mathbf{x})-\alpha_{1}(\mathbf{x})>0$, IV is biased upwards in the presence of mis-classification. Combining the two lemmas yields a well-known bound, namely that $\beta(\mathbf{x})$ lies between the reduced form and IV estimators. Our first result shows that without Assumption 2.2 (non-differential measurement error) these bounds are sharp.

Theorem 2.1. Under Assumptions 2.1 and 2.2(i)-(ii), the sharp identified set is characterized by

$$
\mathbb{E}[y \mid \mathbf{x}, z=k]=c(\mathbf{x})+\beta(\mathbf{x})\left[\frac{p_{k}(\mathbf{x})-\alpha_{0}(\mathbf{x})}{1-\alpha_{0}(\mathbf{x})-\alpha_{1}(\mathbf{x})}\right]
$$

and $\alpha_{0}(\mathbf{x}) \leq p_{k}(\mathbf{x}) \leq 1-\alpha_{1}(\mathbf{x})$ for $k=0,1$ where $p_{k}(\mathbf{x})$ is defined in Equation 4.

Corollary 2.1. Under the conditions of Theorem 2.1, the sharp identified set for $\beta(\mathbf{x})$ is the closed interval between the reduced form estimand $\operatorname{Cov}(y, z \mid \mathbf{x}) / \operatorname{Var}(z \mid \mathbf{x})$ and the IV estimand $\operatorname{Cov}(y, z \mid \mathbf{x}) / \operatorname{Cov}(z, T \mid \mathbf{x})$.

Corollary 2.1 follows by taking differences of the expression for $\mathbb{E}[y \mid \mathbf{x}, z=k]$ across $k=1$ and $k=0$, and substituting the maximum and minimum value for $\alpha_{0}(\mathbf{x})+\alpha_{1}(\mathbf{x})$ consistent with the observed first-stage probabilities. When the mis-classification probabilities are known a priori to satisfy additional restrictions, these bounds can be tightened. ${ }^{5}$ The following corollary collects results for two common cases: one-sided misclassification (either $\alpha_{0}(\mathbf{x})$ or $\alpha_{1}(\mathbf{x})$ equals zero), and symmetric mis-classification $\left(\alpha_{0}(\mathbf{x})=\alpha_{1}(\mathbf{x})\right)$.

Corollary 2.2. Under the conditions of Theorem 2.1, the following restrictions on the misclassification probabilities $\alpha_{0}(\mathbf{x}), \alpha_{1}(\mathbf{x})$ shrink the sharp identified set for $\beta(\mathbf{x})$ to the closed interval between $\Delta \times[\operatorname{Cov}(y, z \mid \mathbf{x}) / \operatorname{Cov}(z, T \mid \mathbf{x})]$ and $\operatorname{Cov}(y, z \mid \mathbf{x}) / \operatorname{Cov}(z, T \mid \mathbf{x})$.

(i) If $\alpha_{0}(\mathbf{x})=0$ then $\Delta=\max _{k} p_{k}(\mathbf{x})$.

(ii) If $\alpha_{1}(\mathbf{x})=0$ then $\Delta=1-\min _{k} p_{k}(\mathbf{x})$.

(iii) If $\alpha_{0}(\mathbf{x})=\alpha_{1}(\mathbf{x})$ then $\Delta=1-2 \min \left\{\min _{k} p_{k}(\mathbf{x}), 1-\max _{k} p_{k}(\mathbf{x})\right\}$.

Theorem 2.1 and Corollaries 2.1-2.2 do not impose Assumption 2.2 (iii) - non-differential measurement error. We now show that this assumption yields further restrictions on the misclassification probabilities $\alpha_{0}(\mathbf{x})$ and $\alpha_{1}(\mathbf{x})$. While these restrictions are more complicated to describe than those from Theorem 2.1, they are straightforward to implement in practice and can be extremely informative, as we will show in our simulation exercises below. To the best of our knowledge, the sharp bounds that we derive by adding Assumption 2.2 (iii) are new to

\footnotetext{
${ }^{5}$ Frazis and Loewenstein (2003) consider a model in which $\alpha_{0}$ and $\alpha_{1}$ do not depend on the exogenous covariates $\mathbf{x}$. In this case $\alpha_{0} \leq \mathbb{P}(T=1 \mid \mathbf{x}, z) \leq 1-\alpha_{1}$ and they suggest minimizing the bounds over $\mathbf{x}$.
} 
the literature. Our result uses two additional conditions to simplify the proof of sharpness. First, we assume that $y$ is continuously distributed. This is natural in an additively separable model and holds in our simulation examples below. Without this assumption, the bounds that we derive are still valid, but may not be sharp. Nevertheless, the reasoning from our proof can be generalized to cases in which $y$ does not have a continuous support set. We also impose $\mathbb{E}[y \mid \mathbf{x}, T=0, z=k] \neq \mathbb{E}[y \mid \mathbf{x}, T=1, z=k]$ for any $k$. This holds generically and is not essential to the proof: it merely simplifies the description of the identified set.

Theorem 2.2. Suppose that the conditional distribution of y given $(\mathbf{x}, T, z)$ is continuous for any values of the conditioning variables and $\mathbb{E}[y \mid \mathbf{x}, T=0, z=k] \neq \mathbb{E}[y \mid \mathbf{x}, T=1, z=k]$ for all $k$. Then, under Assumptions 2.1 and 2.2, the sharp identified set is characterized by Equation 5 from Theorem 2.1 along with $\alpha_{0}(\mathbf{x})<p_{k}(\mathbf{x})<1-\alpha_{1}(\mathbf{x})$ for $k=0,1$ and

$$
\underline{\mu}_{t k}\left(\underline{q}_{t k}\left(\alpha_{0}(\mathbf{x}), \alpha_{1}(\mathbf{x}), \mathbf{x}\right), \mathbf{x}\right) \leq \mu_{k}\left(\alpha_{0}(\mathbf{x}), \mathbf{x}\right) \leq \bar{\mu}_{t k}\left(\bar{q}_{t k}\left(\alpha_{0}(\mathbf{x}), \alpha_{1}(\mathbf{x}), \mathbf{x}\right), \mathbf{x}\right)
$$

for all pairs $(t, k)$ where

$$
\begin{gathered}
\underline{\mu}_{t k}(q, \mathbf{x})=\mathbb{E}[y \mid y \leq q, \mathbf{x}, T=t, z=k], \quad \bar{\mu}_{t k}(q, \mathbf{x})=\mathbb{E}[y \mid y>q, \mathbf{x}, T=t, z=k] \\
\mu_{k}\left(\alpha_{0}(\mathbf{x}), \mathbf{x}\right)=\frac{p_{k}(\mathbf{x}) \mathbb{E}[y \mid \mathbf{x}, z=k, T=1]-\alpha_{0}(\mathbf{x}) \mathbb{E}[y \mid \mathbf{x}, z=k]}{p_{k}(\mathbf{x})-\alpha_{0}(\mathbf{x})}
\end{gathered}
$$

and we define

$$
\begin{aligned}
& \underline{q}_{t k}\left(\alpha_{0}(\mathbf{x}), \alpha_{1}(\mathbf{x}), \mathbf{x}\right)=F_{t k}^{-1}\left(r_{t k}\left(\alpha_{0}(\mathbf{x}), \alpha_{1}(\mathbf{x}), \mathbf{x}\right) \mid \mathbf{x}\right) \\
& \bar{q}_{t k}\left(\alpha_{0}(\mathbf{x}), \alpha_{1}(\mathbf{x}), \mathbf{x}\right)=F_{t k}^{-1}\left(1-r_{t k}\left(\alpha_{0}(\mathbf{x}), \alpha_{1}(\mathbf{x}), \mathbf{x}\right) \mid \mathbf{x}\right)
\end{aligned}
$$

where $F_{t k}^{-1}(\cdot \mid \mathbf{x})$ is the conditional quantile function of $y$ given $(\mathbf{x}, T=t, z=k)$,

$$
\begin{aligned}
& r_{0 k}\left(\alpha_{0}(\mathbf{x}), \alpha_{1}(\mathbf{x}), \mathbf{x}\right)=\frac{\alpha_{1}(\mathbf{x})}{1-p_{k}(\mathbf{x})}\left[\frac{p_{k}(\mathbf{x})-\alpha_{0}(\mathbf{x})}{1-\alpha_{0}(\mathbf{x})-\alpha_{1}(\mathbf{x})}\right] \\
& r_{1 k}\left(\alpha_{0}(\mathbf{x}), \alpha_{1}(\mathbf{x}), \mathbf{x}\right)=\frac{1-\alpha_{1}(\mathbf{x})}{p_{k}(\mathbf{x})}\left[\frac{p_{k}(\mathbf{x})-\alpha_{0}(\mathbf{x})}{1-\alpha_{0}(\mathbf{x})-\alpha_{1}(\mathbf{x})}\right]
\end{aligned}
$$

and $p_{k}(\mathbf{x})$ is defined in Equation 4 .

The intuition for Theorem 2.2 is as follows. For simplicity, suppress dependence on $\mathbf{x}$. Now, fix $(T=t, z=k)$ and $\left(\alpha_{0}, \alpha_{1}\right)$. The observed distribution of $y$ given $(T=t, z=k)$, 
call it $F_{t k}$, is a mixture of two unobserved distributions: the distribution of $y$ given $(T=$ $\left.1, z=k, T^{*}=1\right)$, call it $F_{t k}^{1}$, and the distribution of $y$ given $\left(T=t, z=k, T^{*}=0\right)$, call it $F_{t k}^{0}$. The mixing probabilities are $r_{t k}$ and $1-r_{t k}$ from the statement of Theorem 2.2 and are fully determined by $\left(\alpha_{0}, \alpha_{1}\right)$ and $p_{k}$. Assumptions 2.1 (i) and 2.2 (ii) imply that the unobserved means $\mathbb{E}\left[y \mid T^{*}, T, z\right]$ are fully determined by $\left(\alpha_{0}, \alpha_{1}\right)$ given the observed means $\mathbb{E}[y \mid T, z]$. The question is whether it is possible, given the observed distribution $F_{t k}$, to construct $F_{t k}^{1}$ and $F_{t k}^{0}$ with the required values for $\mathbb{E}\left[y \mid T^{*}, T, z\right]$ such that $F_{t k}=r_{t k} F_{t k}^{1}+\left(1-r_{t k}\right) F_{t k}^{0}$ for all combinations $(t, k)$. If not, then $\left(\alpha_{0}, \alpha_{1}\right)$ does not belong to the identified set. Our proof provides necessary and sufficient conditions for such a mixture to exist at a given point $\left(\alpha_{0}, \alpha_{1}\right)$. We can then appeal to the reasoning from Theorem 2.1 to complete the argument. By ruling out values for $\alpha_{0}$ and $\alpha_{1}$, Theorem 2.2 restricts $\beta$ via Lemma 2.2. While these restrictions can be very informative in practice, they do not yield point identification.

Corollary 2.3. Under Assumptions 2.1 and 2.2 the identified set for $\beta(\mathbf{x})$ contains both the IV estimand $\operatorname{Cov}(y, z \mid \mathbf{x}) / \operatorname{Cov}(z, T \mid \mathbf{x})$ and the true coefficient $\beta(\mathbf{x})$.

Corollary 2.3 follows by Lemma 2.2 because $\alpha_{0}(\mathbf{x})=\alpha_{1}(\mathbf{x})=0$ always belongs to the sharp identified set from Theorem 2.2. Non-differential measurement error cannot exclude the possibility that there is no mis-classification because in this case it is trivial to construct the required mixtures.

Although we focus throughout this paper on the case of a binary instrument, one might wonder whether point identification can be achieved by increasing the support of $z$, perhaps along the lines of Lewbel (2007). The answer turns out to be no. Suppose that we were to modify Assumptions 2.1 and 2.2 to hold for all values of $z$ in some discrete support set. By Lemma 2.2, a binary instrument identifies $\beta(\mathbf{x})$ up to knowledge of the mis-classification probabilities $\alpha_{0}(\mathbf{x})$ and $\alpha_{1}(\mathbf{x})$. It follows that any pair of values $(k, \ell)$ in the support set of $z$ identifies the same object. Accordingly, to identify $\beta(\mathbf{x})$ it is necessary and sufficient to identify the mis-classification probabilities. A binary instrument fails to identify these probabilities because we can never exclude the possibility of zero mis-classification. The same is true of a discrete $K$-valued instrument. Increasing the support of $z$ does, however,

shrink the identified set by increasing the number of restrictions available. If $z$ takes on more than two values, our results in Theorems $2.1-2.2$ continue to apply if " $k=0,1$ " is replaced by "for all $k$. "

\subsection{Point Identification}

The results of the preceding section establish that $\beta(\mathbf{x})$ is not point identified under Assumptions 2.1 and 2.2. In light of this, there are two possible ways to proceed: either one 
can report partial identification bounds based on our characterization of the sharp identified set from Theorem 2.2, or one can attempt to impose stronger assumptions to obtain point identification. In this section we consider the second possibility. We begin by defining the following functions of the model parameters:

$$
\begin{aligned}
& \theta_{1}(\mathbf{x})=\beta(\mathbf{x})\left[1-\alpha_{0}(\mathbf{x})-\alpha_{1}(\mathbf{x})\right]^{-1} \\
& \theta_{2}(\mathbf{x})=\left[\theta_{1}(\mathbf{x})\right]^{2}\left[1+\alpha_{0}(\mathbf{x})-\alpha_{1}(\mathbf{x})\right] \\
& \theta_{3}(\mathbf{x})=\left[\theta_{1}(\mathbf{x})\right]^{3}\left[\left\{1-\alpha_{0}(\mathbf{x})-\alpha_{1}(\mathbf{x})\right\}^{2}+6 \alpha_{0}(\mathbf{x})\left\{1-\alpha_{1}(\mathbf{x})\right\}\right]
\end{aligned}
$$

Now consider the following additional assumption:

Assumption 2.5. $\mathbb{E}\left[\varepsilon^{2} \mid \mathbf{x}, z\right]=\mathbb{E}\left[\varepsilon^{2} \mid \mathbf{x}\right]$

Assumption 2.5 is a second moment version of the standard mean exclusion restriction for the instrument $z$-Assumption 2.1 (iii). It requires that the conditional variance of the error term given the covariates $\mathbf{x}$ does not depend on $z$. Notice that this assumption does not require homoskedasticity with respect to $\mathbf{x}, T^{*}$ or $T$. Assumption 2.5 allows us to derive the following lemma:

Lemma 2.3. Under Assumptions 2.1, 2.2 and 2.5,

$$
\operatorname{Cov}\left(y^{2}, z \mid \mathbf{x}\right)=2 \operatorname{Cov}(y T, z \mid \mathbf{x}) \theta_{1}(\mathbf{x})-\operatorname{Cov}(T, z \mid \mathbf{x}) \theta_{2}(\mathbf{x})
$$

where $\theta_{1}(\mathbf{x})$ and $\theta_{2}(\mathbf{x})$ are defined in Equations 6-\%.

Lemma 2.2 identifies $\theta_{1}(\mathbf{x})$. Since $\operatorname{Cov}(z, T \mid \mathbf{x}) \neq 0$ by Assumption 2.1 (ii), we can solve for $\theta_{2}(\mathbf{x})$ in terms of observables only, using Lemma 2.3. Given knowledge of $\theta_{1}(\mathbf{x})$, we can solve Equation 7 for the difference of mis-classification rates so long as $\beta(\mathbf{x}) \neq 0$.

Corollary 2.4. Under Assumptions 2.1-2.2 and 2.5, $\alpha_{1}(\mathbf{x})-\alpha_{0}(\mathbf{x})$ is identified so long as $\beta(\mathbf{x}) \neq 0$.

Corollary 2.4 identifies the difference of mis-classification error rates. Hence, under onesided mis-classification, $\alpha_{0}(\mathbf{x})=0$ or $\alpha_{1}(\mathbf{x})=0$, augmenting our baseline Assumptions 2.1-2.2 with Assumption 2.5 suffices to identify $\beta(\mathbf{x})$. Notice that $\beta(\mathbf{x})=0$ if and only if $\theta_{1}(\mathbf{x})=0$. Thus, $\beta(\mathbf{x})$ is still identified in the case where Corollary 2.4 fails to apply.

Assumption 2.5 does not suffice to identify $\beta(\mathbf{x})$ without a priori restrictions on the mis-classification error rates. To achieve identification in the general case, we impose the following additional conditions: 


\section{Assumption 2.6.}

(i) $\mathbb{E}\left[\varepsilon^{2} \mid \mathbf{x}, z, T^{*}, T\right]=\mathbb{E}\left[\varepsilon^{2} \mid \mathbf{x}, z, T^{*}\right]$

(ii) $\mathbb{E}\left[\varepsilon^{3} \mid \mathbf{x}, z\right]=\mathbb{E}\left[\varepsilon^{3} \mid \mathbf{x}\right]$

Assumption 2.6 (i) is a second moment version of the non-differential measurement error assumption, Assumption 2.2 (iii). It requires that, given knowledge of $\left(\mathbf{x}, T^{*}, z\right), T$ provides no additional information about the variance of the error term. Note that Assumption 2.6 (i) does not require homoskedasticity of $\varepsilon$ with respect to $\mathbf{x}$ or $T^{*}$. Assumption 2.6 (ii) is a third moment version of Assumption 2.5. It requires that the conditional third moment of the error term given $\mathbf{x}$ does not depend on $z$. This condition neither requires nor excludes skewness in the error term conditional on covariates: it merely states that the skewness is unaffected by the instrument.

While Assumptions 2.5 and 2.6 may appear unfamiliar, we consider them to be fairly natural in the context of an additively separable model in which one has already assumed that $\mathbb{E}[\varepsilon \mid z]=0$ and $\mathbb{E}\left[\varepsilon \mid \mathbf{x}, z, T^{*}, T\right]=\mathbb{E}\left[\mathbf{x}, z, T^{*}\right]$ - Assumptions 2.1 (iii) and 2.2 (iii) from above. ${ }^{6}$ For example, if an applied researcher reports results both for an outcome in logs and levels, she has implicitly assumed independence rather than first moment exclusion. Assumptions 2.1 (iii), 2.5 and 2.6 (ii) are of course implied by $\varepsilon \perp z \mid \mathbf{x}$ while Assumptions 2.2 (iii) and 2.6 (i) are implied by $\varepsilon \perp T \mid\left(\mathbf{x}, T^{*}, z\right)$. Achieving identification via Assumptions 2.5-2.6 involves using information beyond first moments and as such does places higher demands on the data. Assumption 2.6 allows us to derive the following Lemma which, combined with Lemma 2.3, leads to point identification:

Lemma 2.4. Under Assumptions 2.1-2.2 and 2.5-2.6,

$$
\operatorname{Cov}\left(y^{3}, z \mid \mathbf{x}\right)=3 \operatorname{Cov}\left(y^{2} T, z \mid \mathbf{x}\right) \theta_{1}(\mathbf{x})-3 \operatorname{Cov}(y T, z \mid \mathbf{x}) \theta_{2}(\mathbf{x})+\operatorname{Cov}(T, z \mid \mathbf{x}) \theta_{3}(\mathbf{x})
$$

where $\theta_{1}(\mathbf{x}), \theta_{2}(\mathbf{x})$ and $\theta_{3}(\mathbf{x})$ are defined in Equations 6- $\%$.

Theorem 2.3. Under Assumptions 2.1-2.2 and 2.5-2.6, $\beta(\mathbf{x})$ is identified. If $\beta(\mathbf{x}) \neq 0$, then $\alpha_{0}(\mathbf{x})$ and $\alpha_{1}(\mathbf{x})$ are likewise identified.

Lemmas 2.2-2.4 yield a linear system of three equations in $\theta_{1}(\mathbf{x}), \theta_{2}(\mathbf{x})$ and $\theta_{3}(\mathbf{x})$. Under Assumption 2.1 (ii), the system has a unique solution so $\theta_{1}(\mathbf{x}), \theta_{2}(\mathbf{x})$ and $\theta_{3}(\mathbf{x})$ are identified. The proof of Theorem 2.3 shows that, so long as $\beta(\mathbf{x}) \neq 0$, Equations $6-8$ can be solved for $\beta(\mathbf{x}), \alpha_{0}(\mathbf{x})$ and $\alpha_{1}(\mathbf{x})$. If we relax Assumption 2.2 (ii) and assume $\alpha_{0}(\mathbf{x})+\alpha_{1}(\mathbf{x}) \neq 1$ only, $\beta(\mathbf{x})$ is only identified up to sign.

\footnotetext{
${ }^{6}$ If one wishes to weaken our Assumption 2.1 (i) to allow for some form of unobserved heterogeneity, our higher moment assumptions may impose additional restrictions.
} 


\section{Identification-Robust Inference}

We now turn our attention to inference based on the identification results from above. We begin by expressing Lemmas 2.2, 2.3 and 2.4 as unconditional equality moment conditions, and describing the resulting just-identified GMM estimator. As we explain in Section 3.1, inference under binary mis-classification is complicated by problems of weak identification and parameters on the boundary. Section 3.2 provides an overview of our inference procedure. Full details appear in Sections 3.3-3.5. For simplicity we fix the exogenous covariates at some specified level and suppress dependence on $\mathbf{x}$ in the notation. This is appropriate if the covariates have a discrete support. We discuss how to incorporate covariates more generally in Section 3.6.

\subsection{The Non-standard Inference Problem}

Lemmas 2.2-2.4 yield the following system of linear moment equalities in the reduced form parameters $\boldsymbol{\theta}=\left(\theta_{1}, \theta_{2}, \theta_{3}\right)$ from Equations 6-8:

$$
\begin{array}{r}
\operatorname{Cov}(y, z)-\operatorname{Cov}(T, z) \theta_{1}=0 \\
\operatorname{Cov}\left(y^{2}, z\right)-2 \operatorname{Cov}(y T, z) \theta_{1}+\operatorname{Cov}(T, z) \theta_{2}=0 \\
\operatorname{Cov}\left(y^{3}, z\right)-3 \operatorname{Cov}\left(y^{2} T, z\right) \theta_{1}+3 \operatorname{Cov}(y T, z) \theta_{2}-\operatorname{Cov}(T, z) \theta_{3}=0
\end{array}
$$

Non-linearity arises solely through the relationship between the reduced from parameters $\boldsymbol{\theta}$ and the structural parameters $\left(\alpha_{0}, \alpha_{1}, \beta\right)$. To convert the preceding moment equations into unconditional moment equalities, we define the additional reduced form parameters $\boldsymbol{\kappa}=\left(\kappa_{1}, \kappa_{2}, \kappa_{3}\right)$ as follows:

$$
\begin{aligned}
& \kappa_{1}=c-\alpha_{0} \theta_{1} \\
& \kappa_{2}=c^{2}+\sigma_{\varepsilon \varepsilon}+\alpha_{0}\left(\theta_{2}-2 c \theta_{1}\right) \\
& \kappa_{3}=c^{3}+3\left(c-\theta_{1} \alpha_{0}\right) \sigma_{\varepsilon \varepsilon}+\mathbb{E}\left[\varepsilon^{3}\right]-\alpha_{0} \theta_{3}-3 c \alpha_{0}\left[\theta_{1}(c+\beta)-2 \theta_{1}^{2}\left(1-\alpha_{1}\right)\right]
\end{aligned}
$$

Building on this notation, let

$$
\boldsymbol{\psi}_{1}^{\prime}=\left(-\theta_{1}, 1,0,0,0,0\right), \quad \boldsymbol{\psi}_{2}^{\prime}=\left(\theta_{2}, 0,-2 \theta_{1}, 1,0,0\right), \quad \boldsymbol{\psi}_{3}^{\prime}=\left(-\theta_{3}, 0,3 \theta_{2}, 0,-3 \theta_{1}, 1\right)
$$


and collect these in the matrix $\Psi=\left[\begin{array}{lll}\psi_{1} & \boldsymbol{\psi}_{\mathbf{2}} & \boldsymbol{\psi}_{\mathbf{3}}\end{array}\right]$. Defining the observed data vector $\mathbf{w}_{i}^{\prime}=\left(T_{i}, y_{i}, y_{i} T_{i}, y_{i}^{2}, y_{i}^{2} T_{i}, y_{i}^{3}\right)$ for observation $i$, we can re-write the moment equations as:

$$
\mathbb{E}\left[\left(\boldsymbol{\Psi}^{\prime}(\boldsymbol{\theta}) \mathbf{w}_{i}-\boldsymbol{\kappa}\right) \otimes\left(\begin{array}{c}
1 \\
z_{i}
\end{array}\right)\right]=\mathbf{0} .
$$

Equation 10 is a just-identified, linear system of moment equalities in the reduced form parameters $(\boldsymbol{\theta}, \boldsymbol{\kappa})$ and yields explicit GMM estimators $(\widehat{\boldsymbol{\kappa}}, \widehat{\boldsymbol{\theta}})$. From Theorem 2.3, knowledge of $\boldsymbol{\theta}$ suffices to identify $\beta$. From the definitions of $\boldsymbol{\kappa}$ above and $\boldsymbol{\theta}$ in Equations $6-8$, however, the moment equalities from Equation 10 do not depend on $\left(\alpha_{0}, \alpha_{1}\right)$ if $\beta$ equals zero. By continuity, they are nearly uninformative about the mis-classification probabilities if $\beta$ is small. But unless $\beta=0$, knowledge of $\left(\alpha_{0}, \alpha_{1}\right)$ is necessary to recover $\beta$, via Lemma 2.2. Thus, we face a weak identification problem. ${ }^{7}$ Indeed, the GMM estimator of $\widehat{\beta}$ based on Equation 10 may even fail to exist. Using arguments from the proof of Theorem 2.3, this estimator is given by

$$
\widehat{\beta}=\operatorname{sign}\left(\widehat{\theta}_{1}\right) \sqrt{3\left(\widehat{\theta}_{2} / \widehat{\theta}_{1}\right)^{2}-2\left(\widehat{\theta}_{3} / \widehat{\theta}_{1}\right)}
$$

Under our assumptions, $3\left(\theta_{2} / \theta_{1}\right)^{2}>2\left(\theta_{3} / \theta_{1}\right)$ provided that $\beta \neq 0$, but this may not be true of the sample analogue. Indeed, because $\widehat{\theta}_{1}$ appears in the denominator, the terms within the square root will be highly variable if $\beta$ is small. Even if the GMM estimator exists, it may violate the partial identification bounds for $\left(\alpha_{0}, \alpha_{1}\right)$ from Theorem 2.2, or imply that $\left(\alpha_{0}, \alpha_{1}\right)$ are not valid probabilities. Importantly, the partial identification bounds remain informative even if $\beta$ is small or zero: so long as Assumption 2.1 (ii) holds, the first-stage probabilities bound $\alpha_{0}$ and $\alpha_{1}$ from above.

Exactly the same inferential difficulties arise in the case where $T^{*}$ and $z$ are assumed to be jointly exogenous, as in Black et al. (2000); Frazis and Loewenstein (2003); Kane et al. (1999); Lewbel (2007); Mahajan (2006). ${ }^{8}$ This issue, however, has received little attention in the literature. Kane et al. (1999) ensure that $\left(\alpha_{0}, \alpha_{1}\right)$ are valid probabilities by employing a logit specification. Frazis and Loewenstein employ a pseudo-Bayesian approach to ensure that $\alpha_{0}$ and $\alpha_{1}$ are valid probabilities, and to impose partial identification bounds related to those from our Theorem 2.1, i.e. without using the non-differential measurement error restrictions. Because they provide neither simulation evidence nor a theoretical justification for their procedure, however, it is unclear whether this method will yield valid Frequentist coverage. We are unaware of any papers in the related literature that discuss the weak

\footnotetext{
${ }^{7}$ This is essentially equivalent to the problem of estimating mixture probabilities when the means of the component distributions are very similar to each other.

${ }^{8}$ We provide details for Frazis and Loewenstein (2003) and Mahajan (2006) in Appendix C.
} 
identification problem arising when $\beta$ is small.

\subsection{Overview of the Inference Procedure}

In the following sections we develop a procedure for uniformly valid inference in models with a mis-classified binary regressor. Our purpose is to construct a confidence interval for $\beta$ that is robust to possible weak identification, respects the restricted parameter space for $\left(\alpha_{0}, \alpha_{1}\right)$, and incorporates both the information in the equality moment conditions from Equation 10 along with the partial identification bounds from Theorem 2.2. ${ }^{9}$ As argued in the preceding section, our partial identification bounds remain informative even when the equality moment conditions contain essentially no information about $\left(\alpha_{0}, \alpha_{1}\right)$.

To carry out identification-robust inference combining equality and inequality moment conditions, we adopt the generalized moment selection (GMS) approach of Andrews and Soares (2010). This procedure provides a uniformly valid test of a joint null hypothesis for the full parameter vector. In our model, this includes the parameter of interest $\beta$ along with various nuisance parameters: the mis-classification probabilities $\alpha_{0}$ and $\alpha_{1}$, the reduced form parameters $\boldsymbol{\kappa}$, defined in Section 3.1, and a vector $\mathbf{q}$ of parameters that enter the moment inequalities. $^{10}$ Under a given joint null hypothesis for $\left(\beta, \alpha_{0}, \alpha_{1}\right)$, however, $\boldsymbol{\kappa}$ and $\mathbf{q}$ are strongly identified and lie on the interior their respective parameter spaces. Accordingly, in Section 3.4 we explain how to concentrate these parameters out of the GMS procedure, by deriving an appropriate correction to the asymptotic variance matrix for the test. ${ }^{11}$

This leaves us with a uniformly valid test of any joint null hypothesis for $\left(\beta, \alpha_{0}, \alpha_{1}\right)$. To construct a marginal confidence interval for $\beta$ we proceed as follows. Suppose that $z$ is a strong instrument. Then the usual IV estimator provides a valid confidence interval for the reduced from parameter $\theta_{1}$. By Lemma 2.2, knowledge of $\left(1-\alpha_{0}-\alpha_{1}\right)$ suffices to determine $\beta$ from $\theta_{1}$. Thus, a valid confidence interval for $\left(1-\alpha_{0}-\alpha_{1}\right)$ can be combined with the IV interval for $\theta_{1}$ to yield a corresponding interval for $\beta$, via a Bonferroni-type correction. To construct the required interval for $\left(1-\alpha_{0}-\alpha_{1}\right)$, we note from Equations $6-8$ that $\beta$ only enters the moment equality conditions in Equation 10 through $\theta_{1}$. But, again, inference for $\theta_{1}$ is standard provided that $z$ is a strong instrument. We can thus pre-estimate $\theta_{1}$ along with $\boldsymbol{\kappa}$ and $\mathbf{q}$, yielding a uniformly valid GMS test of any joint null hypothesis for $\left(\alpha_{0}, \alpha_{1}\right)$. By inverting this test, we construct a joint confidence set for $\left(\alpha_{0}, \alpha_{1}\right)$ which we then project

\footnotetext{
${ }^{9}$ Note that $\beta=0$ if and only if $\theta_{1}=0$. Thus, if one is merely interested in testing $H_{0}: \beta=0$, one can ignore the mis-classification error problem and test $H_{0}: \theta_{1}=0$ using the standard IV estimator and standard error, provided that $z$ is a strong instrument.

${ }^{10}$ These are defined below in Section 3.3.

${ }^{11}$ Note that we cannot take the same approach to concentrate out $\alpha_{0}$ and $\alpha_{1}$ because the mis-classification probabilities may be weakly identified or lie on the boundary of their parameter space.
} 
to obtain a confidence interval for $\left(1-\alpha_{0}-\alpha_{1}\right)$. Because the parameter space for $\left(\alpha_{0}, \alpha_{1}\right)$ is bounded and two-dimensional, the projection step is computationally trivial. ${ }^{12}$ If desired, one could also carry out a valid test of the null hypothesis that there is no mis-classification, $\alpha_{0}=\alpha_{1}=0$, using the joint test for $\left(\alpha_{0}, \alpha_{1}\right)$. In the following sections we provide full details of our Bonferroni-based confidence interval procedure for $\beta$.

\subsection{Moment Inequalities}

As noted above, the partial identification bounds from Theorems 2.1 and 2.2 remain informative about $\left(\alpha_{0}, \alpha_{1}\right)$ even when $\beta$ is small. To incorporate them in our inference procedure, we first express them as unconditional moment inequalities. The bounds from Theorem 2.1 are given by

$$
p_{k}-\alpha_{0} \geq 0, \quad 1-p_{k}-\alpha_{1} \geq 0, \quad \text { for all } k
$$

where the first-stage probabilities $p_{k}$ are defined in Equation 4. We write these inequalities as

$$
\mathbb{E}\left[m_{1}^{I}\left(\mathbf{w}_{i}, \boldsymbol{\vartheta}\right)\right] \geq \mathbf{0}, \quad m_{1}^{I}\left(\mathbf{w}_{i}, \boldsymbol{\vartheta}\right) \equiv\left[\begin{array}{l}
\left(1-z_{i}\right)\left(T_{i}-\alpha_{0}\right) \\
\left(1-z_{i}\right)\left(1-T_{i}-\alpha_{1}\right) \\
z_{i}\left(T-\alpha_{0}\right) \\
z_{i}\left(1-T_{i}-\alpha_{1}\right)
\end{array}\right]
$$

The bounds derived in Theorem 2.2 by imposing assumption 2.2 (iii) are

$$
\mu_{k}\left(\alpha_{0}\right)-\underline{\mu}_{t k}\left(\underline{q}_{t k}\left(\alpha_{0}, \alpha_{1}\right)\right) \geq 0, \quad \bar{\mu}_{t k}\left(\bar{q}_{t k}\left(\alpha_{0}, \alpha_{1}\right)\right)-\mu_{k}\left(\alpha_{0}\right) \geq 0, \quad \text { for all } t, k
$$

\footnotetext{
${ }^{12}$ We considered two alternatives to the Bonferroni-based inference procedure described here. The first constructs a marginal confidence interval for $\beta$ by projecting a joint confidence set for $\left(\beta, \alpha_{1}, \alpha_{0}\right)$, i.e. without preliminary estimation of $\theta_{1}$. This method is more computationally demanding than our two-dimensional projection and involves a parameter space that is unbounded along the $\beta$-dimension. From a practical perspective, the relevant question is whether the reduction in conservatism from projecting a lower dimensional set is outweighed by the additional conservatism induced by the Bonferroni correction. In our experiments, the full three-dimensional projection and Bonferroni procedure produced broadly similar results: neither reliably dominated in terms of confidence interval width. Given its substantially lower computational burden, we prefer the Bonferroni procedure. We also experimented with two recently proposed methods for sub-vector inference: Kaido et al. (2016) and Bugni et al. (2017). In both cases we obtained significant size distortions, suggesting that our model may not satisfy the regularity conditions required by these papers.
} 
where $\mu_{k}, \underline{\mu}_{t k}, \bar{\mu}_{t k}, \underline{q}_{t k}$ and $\bar{q}_{t k}$ are defined in the statement of the Theorem. Expressing these as unconditional moment inequalities, we have

$$
\mathbb{E}\left[m_{2}^{I}\left(\mathbf{w}_{i}, \boldsymbol{\vartheta}, \mathbf{q}\right)\right] \geq \mathbf{0}, \quad m_{2}^{I}\left(\mathbf{w}_{i}, \boldsymbol{\vartheta}, \mathbf{q}\right) \equiv\left[\begin{array}{c}
m_{2,00}^{I}\left(\mathbf{w}_{i}, \boldsymbol{\vartheta}, \mathbf{q}\right) \\
m_{2,10}^{I}\left(\mathbf{w}_{i}, \boldsymbol{\vartheta}, \mathbf{q}\right) \\
m_{2,01}^{I}\left(\mathbf{w}_{i}, \boldsymbol{\vartheta}, \mathbf{q}\right) \\
m_{2,11}^{I}\left(\mathbf{w}_{i}, \boldsymbol{\vartheta}, \mathbf{q}\right)
\end{array}\right]
$$

where $\mathbf{q} \equiv\left(\underline{q}_{00}, \bar{q}_{00}, \underline{q}_{10}, \bar{q}_{10}, \underline{q}_{01}, \bar{q}_{01}, \underline{q}_{11}, \bar{q}_{11}\right)$ and we define

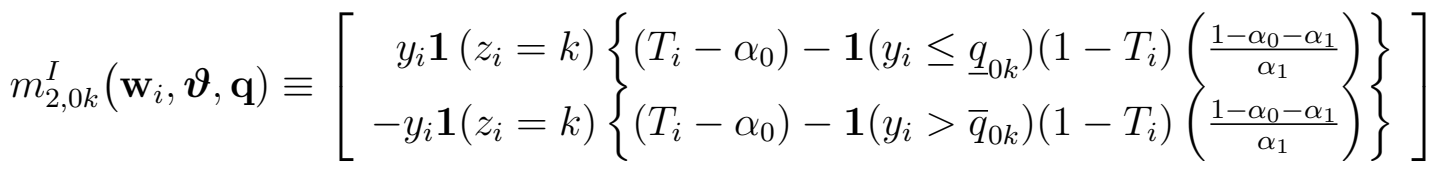

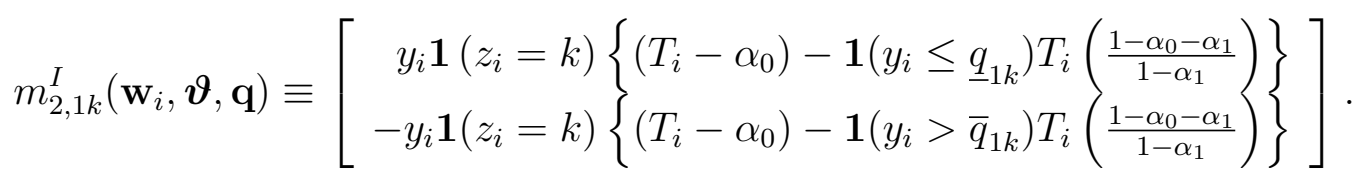

Finally we define $m^{I}=\left(m_{1}^{I^{\prime}}, m_{2}^{I^{\prime}}\right)^{\prime}$. Notice that the second set of inequalities, $m_{2}^{I}$, depends on the unknown parameter $\mathbf{q}$ which is in turn a function of $\left(\alpha_{0}, \alpha_{1}\right)$. In the next section we discuss how $\mathbf{q}$ can be estimated under a given null hypothesis for $\left(\alpha_{0}, \alpha_{1}\right)$.

\subsection{Accounting for Preliminary Estimation}

Let $\boldsymbol{\vartheta}=\left(\alpha_{0}, \alpha_{1}\right)$ and $\boldsymbol{\gamma}=\left(\boldsymbol{\kappa}, \theta_{1}\right)$ where $\theta_{1}$ is defined in Equation 6 and $\boldsymbol{\kappa}$ in Section 3.1. Our moment conditions take the form

$$
\mathbb{E}\left[m^{I}\left(\mathbf{w}_{i}, \boldsymbol{\vartheta}_{0}, \mathbf{q}_{0}\right)\right] \geq \mathbf{0}, \quad \mathbb{E}\left[m^{E}\left(\mathbf{w}_{i}, \boldsymbol{\vartheta}_{0}, \boldsymbol{\gamma}_{0}\right)\right]=\mathbf{0}
$$

where $m^{I}=\left(m_{1}^{I^{\prime}}, m_{2}^{I^{\prime}}\right)^{\prime}$, defined in Section 3.3, and

$$
m^{E}\left(\mathbf{w}_{i}, \boldsymbol{\vartheta}_{0}, \gamma_{0}\right)=\left[\begin{array}{c}
\left\{\boldsymbol{\psi}_{2}^{\prime}\left(\theta_{1}, \alpha_{0}, \alpha_{1}\right) \mathbf{w}_{i}-\kappa_{2}\right\} z_{i} \\
\left\{\boldsymbol{\psi}_{3}^{\prime}\left(\theta_{1}, \alpha_{0}, \alpha_{1}\right) \mathbf{w}_{i}-\kappa_{3}\right\} z_{i}
\end{array}\right]
$$

Notice that we now write $\boldsymbol{\psi}_{2}$ and $\boldsymbol{\psi}_{3}$, defined in Equation 9, as explicit functions of $\left(\theta_{1}, \alpha_{0}, \alpha_{1}\right)$, using the definitions of $\left(\theta_{2}, \theta_{3}\right)$ from Equations 7-8. To construct a GMS test of the null hypothesis $H_{0}: \boldsymbol{\vartheta}=\boldsymbol{\vartheta}_{\mathbf{0}}$ based on Equation 15, we require preliminary estimators $\widehat{\gamma}\left(\boldsymbol{\vartheta}_{0}\right)$ and $\widehat{\mathbf{q}}\left(\boldsymbol{\vartheta}_{0}\right)$ that are consistent and asymptotically normal under the null. We now provide full details of the construction and derive the associated adjustment to the asymptotic variance 
matrix.

Consider first the equality moment conditions $m^{E}$. For these we require preliminary estimators of $\theta_{1}, \kappa_{2}$, and $\kappa_{3}$. Recall that $\theta_{1}$ is simply the IV estimand: it can be consistently estimated directly from observations of $(y, T, z)$ without knowledge of $\alpha_{0}$ or $\alpha_{1}$. Note, moreover, from Equation 10 that $\boldsymbol{\kappa}$ is simply a vector of intercepts. These can be directly estimated from observations of $\mathbf{w}$ because $\boldsymbol{\Psi}\left(\theta_{1}, \alpha_{0}, \alpha_{1}\right)$ is consistently estimable under the null $H_{0}: \boldsymbol{\vartheta}=\boldsymbol{\vartheta}_{0}$ : the hypothesis specifies $\alpha_{0}$ and $\alpha_{1}$ and IV provides a consistent estimator of $\theta_{1}$. Accordingly, define

$$
h^{E}\left(\mathbf{w}_{i}, \boldsymbol{\vartheta}, \boldsymbol{\gamma}\right)=\left[\begin{array}{c}
\boldsymbol{\Psi}^{\prime}\left(\theta_{1}, \alpha_{0}, \alpha_{1}\right) \mathbf{w}_{i}-\boldsymbol{\kappa} \\
\left\{\boldsymbol{\psi}_{1}^{\prime}\left(\theta_{1}\right) \mathbf{w}_{i}-\kappa_{1}\right\} z_{i}
\end{array}\right]
$$

Under $H_{0}: \boldsymbol{\vartheta}=\boldsymbol{\vartheta}_{0}$, the just-identified GMM-estimator based on $\mathbb{E}\left[h^{E}\left(\mathbf{w}_{i}, \boldsymbol{\vartheta}_{0}, \boldsymbol{\gamma}_{0}\right)\right]=\mathbf{0}$ yields a consistent and asymptotically normal estimator of $\gamma_{0}$ under the usual regularity conditions.

Now consider the inequality moment conditions $m^{I}$. From Section 3.3 we see that $m_{2}^{I}$ depends on $\mathbf{q}$, the vector of conditional quantiles $\bar{q}_{t k}$ and $\underline{q}_{t k}$ defined in Theorem 2.2. Under the assumption that $y$ follows a continuous distribution, as maintained in Theorem 2.2, these can be expressed as conditional moment equalities as follows:

$$
\begin{aligned}
& \mathbb{E}\left[\mathbf{1}\left(y \leq \underline{q}_{t k}\right) \mid T=t, z=k\right]-r_{t k}\left(\alpha_{0}, \alpha_{1}\right)=0 \\
& \mathbb{E}\left[\mathbf{1}\left(y \leq \bar{q}_{t k}\right) \mid T=t, z=k\right]-\left(1-r_{t k}\left(\alpha_{0}, \alpha_{1}\right)\right)=0
\end{aligned}
$$

where $r_{t k}$ is defined in Theorem 2.2 and $t, k=0,1$. Under $H_{0}: \boldsymbol{\vartheta}=\boldsymbol{\vartheta}_{0}$, a consistent estimator $\widehat{r}_{t k}$ of $r_{t k}$ can be obtained directly from $\widehat{p}_{k}$, the sample analogue of $p_{k}$ based on iid observations of $\mathbf{w}_{i}$. In turn, the $\left(\widehat{r}_{t k}\right)^{\text {th }}$ and $\left(1-\widehat{r}_{t k}\right)^{\text {th }}$ sample conditional quantiles of $y$ provide consistent estimates of $\underline{q}_{t k}$ and $\bar{q}_{t k} \cdot{ }^{13}$ Collecting these for all $(t, k)$ gives $\widehat{\mathbf{q}}\left(\boldsymbol{\vartheta}_{0}\right)$. Now, define

$$
h^{I}\left(\mathbf{w}_{i}, \boldsymbol{\vartheta}, \mathbf{q}\right)=\left[\begin{array}{c}
h_{0}^{I}(\mathbf{w}, \boldsymbol{\vartheta}, \mathbf{q}) \\
h_{1}^{I}(\mathbf{w}, \boldsymbol{\vartheta}, \mathbf{q})
\end{array}\right]
$$

\footnotetext{
${ }^{13}$ Consistency of the sample quantiles requires $0<r_{t k}<1$. If $r_{t k}=0$ or 1 for some $(t, k)$, however, then the associated moment inequality is trivially satisfied and we no longer require estimates of $\underline{q}_{t k}, \bar{q}_{t k}$.
} 
where

$$
h_{k}^{I}\left(\mathbf{w}_{i}, \boldsymbol{\vartheta}, \mathbf{q}\right)=\left[\begin{array}{l}
\mathbf{1}\left(y_{i} \leq \underline{q}_{0 k}\right) \mathbf{1}\left(z_{i}=k\right)\left(1-T_{i}\right)-\left(\frac{\alpha_{1}}{1-\alpha_{0}-\alpha_{1}}\right) \mathbf{1}\left(z_{i}=k\right)\left(T_{i}-\alpha_{0}\right) \\
\mathbf{1}\left(y_{i} \leq \bar{q}_{0 k}\right) \mathbf{1}\left(z_{i}=k\right)\left(1-T_{i}\right)-\left(\frac{1-\alpha_{0}}{1-\alpha_{0}-\alpha_{1}}\right) \mathbf{1}\left(z_{i}=k\right)\left(1-T_{i}-\alpha_{1}\right) \\
\mathbf{1}\left(y_{i} \leq \underline{q}_{1 k}\right) \mathbf{1}\left(z_{i}=k\right) T_{i}-\left(\frac{1-\alpha_{1}}{1-\alpha_{0}-\alpha_{1}}\right) \mathbf{1}\left(z_{i}=k\right)\left(T_{i}-\alpha_{0}\right) \\
\mathbf{1}\left(y_{i} \leq \bar{q}_{1 k}\right) \mathbf{1}\left(z_{i}=k\right) T_{i}-\left(\frac{\alpha_{0}}{1-\alpha_{0}-\alpha_{1}}\right) \mathbf{1}\left(z_{i}=k\right)\left(1-T_{i}-\alpha_{1}\right)
\end{array}\right] .
$$

Equation 21 gives the unconditional version of Equations 18-19. Now, under the null $\widehat{\mathbf{q}}\left(\boldsymbol{\vartheta}_{0}\right)$ converges in probability to $\mathbf{q}_{0}$, which satisfies the just-identified collection of moment equalities $\mathbb{E}\left[h^{I}\left(\mathbf{w}_{i}, \boldsymbol{\vartheta}_{0}, \mathbf{q}_{0}\right)\right]=\mathbf{0}$. Although $h^{I}$ is a discontinuous function of $\mathbf{q}$, it is bounded for any fixed $\left(\alpha_{0}, \alpha_{1}\right)$. Moreover, since $y \mid(T=t, z=k)$ is a continuous random variable, $\mathbb{E}\left[h^{I}\left(\mathbf{w}_{i}, \boldsymbol{\vartheta}, \mathbf{q}\right)\right]$ is continuously differentiable with respect to $\mathbf{q}$. Hence, $\widehat{\mathbf{q}}$ is asymptotically normal under mild regularity conditions. ${ }^{14}$ To account for the effect of preliminary estimation of $\mathbf{q}$ and $\gamma$ on the asymptotic variance matrix used in the GMS test, we rely on the following Lemma:

Lemma 3.1. Let $\bar{m}_{1, n}^{I}(\boldsymbol{\vartheta})=n^{-1} \sum_{i=1}^{n} m_{1, n}^{I}\left(\mathbf{w}_{i}, \boldsymbol{\vartheta}\right)$ and define $\bar{m}_{2, n}^{I}, \bar{m}_{n}^{E}, \bar{h}_{n}^{I}, \bar{h}_{n}^{E}$ analogously. Further let $\widehat{\boldsymbol{\gamma}}\left(\boldsymbol{\vartheta}_{0}\right)=\arg \min _{\boldsymbol{\gamma}}\left\|\bar{h}_{n}^{E}\left(\boldsymbol{\vartheta}_{0}, \boldsymbol{\gamma}\right)\right\|$ and $\left\|h_{n}^{I}\left(\boldsymbol{\vartheta}_{0}, \widehat{\mathbf{q}}\left(\boldsymbol{\vartheta}_{0}\right)\right)\right\| \leq \inf _{\mathbf{q}}\left\|h_{n}^{I}\left(\boldsymbol{\vartheta}_{0}, \mathbf{q}\right)\right\|+o_{p}(1)$. Then, under standard regularity conditions

$$
\sqrt{n}\left[\begin{array}{l}
\bar{m}_{1, n}^{I}\left(\boldsymbol{\vartheta}_{0}\right) \\
\bar{m}_{2, n}^{I}\left(\boldsymbol{\vartheta}_{0}, \widehat{\mathbf{q}}\left(\boldsymbol{\vartheta}_{0}\right)\right) \\
\bar{m}_{n}^{E}\left(\boldsymbol{\vartheta}_{0}, \widehat{\gamma}\left(\boldsymbol{\vartheta}_{0}\right)\right)
\end{array}\right] \rightarrow_{p}\left[\begin{array}{ccccc}
\mathbf{I} & \mathbf{0} & \mathbf{0} & \mathbf{0} & \mathbf{0} \\
\mathbf{0} & \mathbf{I} & \mathbf{0} & B^{I}\left(\boldsymbol{\vartheta}_{0}, \boldsymbol{q}_{0}\right) & \mathbf{0} \\
\mathbf{0} & \mathbf{0} & \mathbf{I} & \mathbf{0} & B^{E}\left(\boldsymbol{\vartheta}_{0}, \boldsymbol{\gamma}_{0}\right)
\end{array}\right] \sqrt{n}\left[\begin{array}{l}
\bar{m}_{1, n}^{I}\left(\boldsymbol{\vartheta}_{0}\right) \\
\bar{m}_{2, n}^{I}\left(\boldsymbol{\vartheta}_{0}, \mathbf{q}_{0}\right) \\
\bar{m}_{n}^{E}\left(\boldsymbol{\vartheta}_{0}, \boldsymbol{\gamma}_{0}\right) \\
\bar{h}_{n}^{I}\left(\boldsymbol{\vartheta}_{0}, \mathbf{q}_{0}\right) \\
\bar{h}_{n}^{E}\left(\boldsymbol{\vartheta}_{0}, \boldsymbol{\gamma}_{0}\right)
\end{array}\right]
$$

where we define $B^{I}(\boldsymbol{\vartheta}, \boldsymbol{q})=\left(1-\alpha_{0}-\alpha_{1}\right)[\operatorname{diag}(\mathbf{a})]^{-1} \mathbf{q}$ and $B^{E}(\boldsymbol{\vartheta}, \boldsymbol{\gamma})=-M^{E}\left(H^{E}\right)^{-1}$ with $\mathbf{a}^{\prime}=\left(\alpha_{1}, \alpha_{1}, 1-\alpha_{1}, 1-\alpha_{1}, \alpha_{1}, \alpha_{1}, 1-\alpha_{1}, 1-\alpha_{1}\right)$, and

$$
M^{E}=\left[\begin{array}{cccc}
0 & -\mathbb{E}\left[z_{i}\right] & 0 & \left(\frac{\partial \boldsymbol{\psi}_{2}}{\partial \theta_{1}}\right)^{\prime} \mathbb{E}\left[\mathbf{w}_{i} z_{i}\right] \\
0 & 0 & -\mathbb{E}\left[z_{i}\right] & \left(\frac{\partial \boldsymbol{\psi}_{3}}{\partial \theta_{1}}\right)^{\prime} \mathbb{E}\left[\mathbf{w}_{i} z_{i}\right]
\end{array}\right], H^{E}=\left[\begin{array}{cccc}
-1 & 0 & 0 & \left(\frac{\partial \boldsymbol{\psi}_{1}}{\partial \theta_{1}}\right)^{\prime} \mathbb{E}\left[\mathbf{w}_{i}\right] \\
0 & -1 & 0 & \left(\frac{\partial \boldsymbol{\psi}_{2}}{\partial \theta_{1}}\right)^{\prime} \mathbb{E}\left[\mathbf{w}_{i}\right] \\
0 & 0 & -1 & \left(\frac{\partial \boldsymbol{\psi}_{3}}{\partial \theta_{1}}\right)^{\prime} \mathbb{E}\left[\mathbf{w}_{i}\right] \\
-\mathbb{E}\left[z_{i}\right] & 0 & 0 & \left(\frac{\partial \boldsymbol{\psi}_{1}}{\partial \theta_{1}}\right)^{\prime} \mathbb{E}\left[\mathbf{w}_{i} z_{i}\right]
\end{array}\right] .
$$

Lemma 3.1 relates the sample analogues $\bar{m}_{2, n}^{I}$ and $\bar{m}_{n}^{E}$ evaluated at the preliminary esti-

\footnotetext{
${ }^{14}$ For details, see Andrews (1994) and Newey and McFadden (1994) Section 7.
} 
mators $\widehat{\mathbf{q}}\left(\boldsymbol{\vartheta}_{0}\right)$ and $\widehat{\boldsymbol{\gamma}}\left(\boldsymbol{\vartheta}_{0}\right)$ to their counterparts evaluated at the true parameter values $\mathbf{q}_{0}$ and $\boldsymbol{\gamma}_{0}$. The estimator $\widehat{\boldsymbol{\gamma}}\left(\boldsymbol{\vartheta}_{0}\right)$ exactly solves $h_{n}^{E}\left(\boldsymbol{\vartheta}_{0}, \boldsymbol{\gamma}\right)=0$ while $\widehat{q}\left(\boldsymbol{\vartheta}_{0}\right)$, constructed as described immediately before the statement of the Lemma, approximately solves $\bar{h}_{n}^{I}\left(\boldsymbol{\vartheta}_{0}, \mathbf{q}\right)=0$. A few lines of matrix algebra show that the determinant of $H^{E}$ equals $\operatorname{Cov}(z, T)$. Hence, $B^{E}$ is well-defined if $z$ is a relevant instrument. The matrix $B^{I}$ is likewise well-defined provided that $\alpha_{1} \neq 0$ and the elements of $\mathbf{q}_{0}$ are computed for probabilities strictly between zero and one. If either of these conditions fails, however, some of the moment inequalities in $m_{2}^{I}$ are trivially satisfied and can be dropped (see Footnote 13). After removing the corresponding elements of $\mathbf{q}_{0}$ and $\mathbf{a}, B^{I}$ becomes well-defined. The regularity conditions required for Lemma 3.1 are mild. The result relies on a number of mean-value expansions: $\bar{h}_{n}^{E}\left(\boldsymbol{\vartheta}_{0}, \boldsymbol{\gamma}_{0}\right)$ and $\bar{m}_{n}^{E}\left(\boldsymbol{\vartheta}_{0}, \boldsymbol{\gamma}_{0}\right)$ are expanded around $\boldsymbol{\gamma}=\widehat{\boldsymbol{\gamma}}\left(\boldsymbol{\vartheta}_{0}\right)$ while $\mathbb{E}\left[h^{I}\left(\mathbf{w}_{i}, \boldsymbol{\vartheta}_{0}, \mathbf{q}_{0}\right)\right]$ and $\mathbb{E}\left[m_{2}^{I}\left(\mathbf{w}_{i}, \boldsymbol{\vartheta}_{0}, \mathbf{q}_{0}\right)\right]$ are expanded around $\mathbf{q}=\widehat{\mathbf{q}}\left(\boldsymbol{\vartheta}_{0}\right)$. These expansions, in turn, rely on the fact that $\mathbf{q}$ and $\boldsymbol{\gamma}$ are interior to their respective parameter spaces and the relevant functions are all continuously differentiable in our example.

We now have all the ingredients required to construct an asymptotic variance matrix for the GMS test that accounts for preliminary estimation of $\boldsymbol{\gamma}$ and $\mathbf{q}$. Let $m^{\prime}=\left(m_{1}^{I^{\prime}}, m_{2}^{I^{\prime}}, m^{E^{\prime}}\right)$, $h^{\prime}=\left(h^{I^{\prime}}, h^{E^{\prime}}\right)$, and define the shorthand $\boldsymbol{\tau}_{0}^{\prime}=\left(\boldsymbol{\gamma}_{0}^{\prime}, \mathbf{q}_{0}^{\prime}\right)$ and $\widehat{\boldsymbol{\tau}}_{0}^{\prime}=\left(\widehat{\boldsymbol{\gamma}}^{\prime}\left(\boldsymbol{\vartheta}_{0}\right), \widehat{\mathbf{q}}^{\prime}\left(\boldsymbol{\vartheta}_{0}\right)\right)$. Given a collection of iid observations $\left(\mathbf{w}_{1}, \ldots, \mathbf{w}_{n}\right)$, we have

$$
\sqrt{n}\left[\begin{array}{c}
\bar{m}_{n}\left(\boldsymbol{\vartheta}_{0}, \boldsymbol{\tau}_{0}\right) \\
\bar{h}_{n}\left(\boldsymbol{\vartheta}_{0}, \boldsymbol{\tau}_{0}\right)
\end{array}\right] \rightarrow_{d} N\left(\mathbf{0}, \mathcal{V}\left(\boldsymbol{\vartheta}_{0}, \boldsymbol{\tau}_{0}\right)\right), \quad \mathcal{V}\left(\boldsymbol{\vartheta}_{0}, \boldsymbol{\tau}_{0}\right)=\operatorname{Var}\left[\begin{array}{c}
m\left(\mathbf{w}_{i}, \boldsymbol{\vartheta}_{0}, \boldsymbol{\tau}_{0}\right) \\
h\left(\mathbf{w}_{i}, \boldsymbol{\vartheta}_{0}, \boldsymbol{\tau}_{0}\right)
\end{array}\right]
$$

under $H_{0}: \boldsymbol{\vartheta}=\boldsymbol{\vartheta}_{0}$, by an appropriate central limit theorem. What we require for the test, however, is the asymptotic variance matrix of $\sqrt{n} \bar{m}_{n}\left(\boldsymbol{\vartheta}_{0}, \widehat{\boldsymbol{\tau}}_{0}\right)$. Combining Equation 22 with Lemma 3.1, we obtain

$$
\operatorname{Avar}\left(\sqrt{n} \bar{m}_{n}\left(\boldsymbol{\vartheta}_{0}, \widehat{\boldsymbol{\tau}}_{0}\right)\right)=\Xi\left(\boldsymbol{\vartheta}_{0}, \boldsymbol{\tau}_{0}\right) \mathcal{V}\left(\boldsymbol{\vartheta}_{0}, \boldsymbol{\tau}_{0}\right) \Xi^{\prime}\left(\boldsymbol{\vartheta}_{0}, \boldsymbol{\tau}_{0}\right)
$$

with

$$
\Xi(\boldsymbol{\vartheta}, \boldsymbol{\tau})=\left[\begin{array}{ll}
\mathbf{I} & B(\boldsymbol{\vartheta}, \boldsymbol{\tau})
\end{array}\right], \quad B(\boldsymbol{\vartheta}, \boldsymbol{\tau})=\left[\begin{array}{cc}
\mathbf{0} & \mathbf{0} \\
B^{I}(\boldsymbol{\vartheta}, \mathbf{q}) & \mathbf{0} \\
\mathbf{0} & B^{E}(\boldsymbol{\vartheta}, \boldsymbol{\gamma})
\end{array}\right]
$$

where $B^{I}(\cdot, \cdot)$ and $B^{E}(\cdot, \cdot)$ are defined in Lemma 3.1. Finally, we construct a consistent estimator $\widehat{\Sigma}_{n}\left(\boldsymbol{\vartheta}_{0}\right)$ of the asymptotic variance matrix of $\sqrt{n} \bar{m}_{n}\left(\boldsymbol{\vartheta}_{0}, \widehat{\boldsymbol{\tau}}_{0}\right)$ under the null:

$$
\widehat{\Sigma}_{n}\left(\boldsymbol{\vartheta}_{0}\right) \equiv \Xi\left(\boldsymbol{\vartheta}_{0}, \widehat{\boldsymbol{\tau}}_{0}\right) \widehat{\mathcal{V}}_{n}\left(\boldsymbol{\vartheta}_{0}, \widehat{\boldsymbol{\tau}}\right) \Xi^{\prime}\left(\boldsymbol{\vartheta}_{0}, \widehat{\boldsymbol{\tau}}_{0}\right)
$$


where

$$
\widehat{\mathcal{V}}_{n}(\boldsymbol{\vartheta}, \boldsymbol{\tau}) \equiv \frac{1}{n} \sum_{i=1}^{n}\left[\begin{array}{c}
m\left(\mathbf{w}_{i}, \boldsymbol{\vartheta}, \boldsymbol{\tau}\right)-\bar{m}_{n}(\boldsymbol{\vartheta}, \boldsymbol{\tau}) \\
h\left(\mathbf{w}_{i}, \boldsymbol{\vartheta}, \boldsymbol{\tau}\right)-\bar{h}_{n}(\boldsymbol{\vartheta}, \boldsymbol{\tau})
\end{array}\right]\left[\begin{array}{c}
m\left(\mathbf{w}_{i}, \boldsymbol{\vartheta}, \boldsymbol{\tau}\right)-\bar{m}_{n}(\boldsymbol{\vartheta}, \boldsymbol{\tau}) \\
h\left(\mathbf{w}_{i}, \boldsymbol{\vartheta}, \boldsymbol{\tau}\right)-\bar{h}_{n}(\boldsymbol{\vartheta}, \boldsymbol{\tau})
\end{array}\right]^{\prime}
$$

In the following section we provide a step-by-step description of our inference procedure.

\subsection{Details of the Inference Procedure}

In this section we provide full details of our Bonferroni-based inference procedure. We begin by defining some notation. Let $J$ denote the total number of inequality moment conditions, $K$ denote the total number of equality moment conditions, and define

$$
\bar{m}_{n}(\boldsymbol{\vartheta}, \boldsymbol{\tau})=\left[\begin{array}{l}
\bar{m}_{n}^{I}(\boldsymbol{\vartheta}, \mathbf{q}) \\
\bar{m}_{n}^{E}(\boldsymbol{\vartheta}, \boldsymbol{\gamma})
\end{array}\right]=\left[\begin{array}{c}
\bar{m}_{n, 1}^{I}(\boldsymbol{\vartheta}) \\
\bar{m}_{n, 2}^{I}(\boldsymbol{\vartheta}, \mathbf{q}) \\
\bar{m}_{n}^{E}(\boldsymbol{\vartheta}, \boldsymbol{\gamma})
\end{array}\right]=\frac{1}{n} \sum_{i=1}^{n}\left[\begin{array}{l}
m_{1}^{I}\left(\mathbf{w}_{i}, \boldsymbol{\vartheta}\right) \\
m_{2}^{I}\left(\mathbf{w}_{i}, \boldsymbol{\vartheta}, \mathbf{q}\right) \\
m^{E}\left(\mathbf{w}_{i}, \boldsymbol{\vartheta}, \boldsymbol{\gamma}\right)
\end{array}\right]
$$

with $m_{1}^{I}$ as defined in Equation 11, $m_{2}^{I}$ in Equations $12-14$ and $m^{E}$ in Equation $16 .^{15}$ Now let $S$ be the function

$$
S(\mathbf{x}, \mathbf{y})=\sum_{j} \min \left\{0, x_{j}^{2}\right\}+\mathbf{y}^{\prime} \mathbf{y}
$$

where $\mathbf{x}, \mathbf{y}$ are two finite-dimensional real vectors and $x_{j}$ denotes the $j^{\text {th }}$ element of $\mathbf{x}$. This function will be used to calculate the modified method of moments (MMM) test statistic as part of the GMS test below. The argument $\mathbf{x}$ stands in for the moment inequalities, which only contribute to the test statistic when they are violated, i.e. take on a negative value. Using this notation, we now detail the first step of our inference procedure: a GMS test for $\boldsymbol{\vartheta}=\left(\alpha_{0}, \alpha_{1}\right)$ with preliminary estimation of $\mathbf{q}$ and $\boldsymbol{\gamma}$ under the null.

Algorithm 3.1 (GMS Test for $\alpha_{0}$ and $\alpha_{1}$ ).

Inputs: hypothesis $\boldsymbol{\vartheta}_{0}$; iid dataset $\left\{\mathbf{w}_{i}\right\}_{i=1}^{n}$; simulations $\left\{\boldsymbol{\zeta}^{(r)}\right\}_{r=1}^{R} \sim$ iid $N_{J+K}(\mathbf{0}, \mathbf{I})$.

1. Calculate the variance matrix estimator $\widehat{\Sigma}_{n}\left(\boldsymbol{\vartheta}_{0}\right)$.

(i) Calculate $\widehat{\boldsymbol{\tau}}_{0}=\left(\widehat{\mathbf{q}}_{0}^{\prime}, \widehat{\boldsymbol{\gamma}}_{0}^{\prime}\right)^{\prime}$ where $\widehat{\boldsymbol{\gamma}}_{0}=\widehat{\boldsymbol{\gamma}}\left(\boldsymbol{\vartheta}_{0}\right)$ and $\widehat{\mathbf{q}}_{0}=\widehat{\mathbf{q}}\left(\boldsymbol{\vartheta}_{0}\right)$ from Section 3.4.

(ii) Calculate $\Xi\left(\boldsymbol{\vartheta}_{0}, \widehat{\boldsymbol{\tau}}_{0}\right)$ using Equation 24.

(iii) Calculate $\widehat{\mathcal{V}}_{n}\left(\boldsymbol{\vartheta}_{0}, \widehat{\boldsymbol{\tau}}_{0}\right)$ using Equation 26.

(iv) Set $\widehat{\Sigma}_{n}\left(\boldsymbol{\vartheta}_{0}\right)=\Xi\left(\boldsymbol{\vartheta}_{0}, \widehat{\boldsymbol{\tau}}_{0}\right) \widehat{\mathcal{V}}_{n}\left(\boldsymbol{\vartheta}_{0}, \widehat{\boldsymbol{\tau}}\right) \Xi^{\prime}\left(\boldsymbol{\vartheta}_{0}, \widehat{\boldsymbol{\tau}}_{0}\right)$.

\footnotetext{
${ }^{15}$ In our problem $K=2$ and $J$ is at most 12 . Under certain nulls for $\left(\alpha_{0}, \alpha_{1}\right)$, however, we drop components of $m_{2}^{I}$ as they are trivially satisfied. See footnote 13 and Section 3.4 for further discussion.
} 
2. Calculate the test statistic $T_{n}\left(\boldsymbol{\vartheta}_{0}\right)$.

(i) Calculate $\sqrt{n} \bar{m}_{n}\left(\boldsymbol{\vartheta}_{0}, \widehat{\boldsymbol{\tau}}_{0}\right)$ using Equation 27.

(ii) Set $\boldsymbol{\nu}_{n}\left(\boldsymbol{\vartheta}_{0}\right)=\left[\operatorname{diag}\left\{\widehat{\Sigma}_{n}\left(\boldsymbol{\vartheta}_{0}\right)\right\}\right]^{-1 / 2}\left[\sqrt{n} \bar{m}_{n}\left(\boldsymbol{\vartheta}_{0}, \widehat{\boldsymbol{\tau}}_{0}\right)\right]$.

(iii) Let $\boldsymbol{\nu}_{n}^{I}\left(\boldsymbol{\vartheta}_{0}\right)$ denote the first $J$ elements of $\boldsymbol{\nu}_{n}$ and $\boldsymbol{\nu}_{n}^{E}\left(\boldsymbol{\vartheta}_{0}\right)$ the last $K$ elements.

(iv) Set $T_{n}\left(\boldsymbol{\vartheta}_{0}\right)=S\left(\boldsymbol{\nu}_{n}^{I}\left(\boldsymbol{\vartheta}_{0}\right), \boldsymbol{\nu}_{n}^{E}\left(\boldsymbol{\vartheta}_{0}\right)\right)$ using Equation 28.

3. Construct the moment selection matrix $\Phi$.

(i) For $j=1, \ldots, J$ set $\varphi_{j}^{I}=\mathbf{1}\left\{\nu_{n, j}^{I}\left(\boldsymbol{\vartheta}_{0}\right) \leq \sqrt{\log n}\right\}$ and let $\widetilde{J}=\sum_{j=1}^{J} \varphi_{j}^{I}$.

(ii) For $j=1, \ldots, K$ set $\varphi_{j}^{E}=1$.

(iii) Set $\boldsymbol{\varphi}=\left(\varphi_{1}^{I}, \ldots, \varphi_{J}^{I}, \varphi_{1}^{E}, \ldots, \varphi_{K}^{E}\right)^{\prime}$.

(iv) Let $\Phi$ be the $(\widetilde{J}+K) \times(J+K)$ of zeros and ones that selects those elements $x_{j}$ of an arbitrary vector $\mathbf{x}$ that correspond to $\varphi_{j}=1$.

4. Simulate the sampling distribution of $T_{n}\left(\boldsymbol{\vartheta}_{0}\right)$ under the null.

(i) Let $\widehat{\Omega}$ be the correlation matrix that corresponds to $\widehat{\Sigma}_{n}\left(\boldsymbol{\vartheta}_{0}\right)$.

(ii) For each $r=1, \ldots, R$ set $\boldsymbol{\xi}^{(r)}=\left[\Phi \widehat{\Omega} \Phi^{\prime}\right]^{1 / 2} \Phi \boldsymbol{\zeta}^{(r)}$.

(iii) Let $\boldsymbol{\xi}_{I}^{(r)}$ denote the first $\widetilde{J}$ and $\boldsymbol{\xi}_{E}^{(r)}$ the last $K$ elements of $\boldsymbol{\xi}^{(r)}$.

(iv) For each $r=1, \ldots, R$ set $T_{n}^{(r)}\left(\boldsymbol{\vartheta}_{0}\right)=S\left(\boldsymbol{\xi}_{I}^{(r)}, \boldsymbol{\xi}_{E}^{(r)}\right)$ using Equation 28.

5. Calculate the p-value of the test: $\widehat{p}\left(\boldsymbol{\vartheta}_{0}\right)=\frac{1}{R} \sum_{r=1}^{R} \mathbf{1}\left\{T_{n}^{(r)}\left(\boldsymbol{\vartheta}_{0}\right)>T_{n}\left(\boldsymbol{\vartheta}_{0}\right)\right\}$.

Algorithm 3.1 corresponds to the asymptotic version of the GMS test from Andrews and Soares (2010), based on the MMM test statistic - $S_{1}$ in Andrews and Soares (2010) - and the "BIC choice" $\kappa_{n}=\sqrt{\log n}$ for the sequence of constants $\kappa_{n}$ used for moment selection. The procedure is as follows. In Step 1, we compute a consistent estimator of the asymptotic variance matrix of the full set of moment conditions, under the null, accounting for preliminary estimation of $\mathbf{q}$ and $\gamma$ as explained in Section 3.4. In step 2, we calculate the observed value of the MMM test statistic. Note that this test statistic uses only the diagonal elements of $\widehat{\Sigma}_{n}\left(\boldsymbol{\vartheta}_{0}\right)$. Moreover, the moment inequalities only contribute to $T_{n}$ if they are violated, i.e. if they take on a negative value. In step 3 we determine which moment inequalities are "far from binding," defined as having a t-ratio greater than $\sqrt{\log n}$. These moment inequalities will be excluded when approximating the large-sample distribution of 
the test statistic. The matrix $\Phi$ encodes the results of the moment selection step. Premultiplying a $(J+K)$-vector $\mathbf{x}$ by $\Phi$ results in a $(\widetilde{J} \times K)$-vector $\widetilde{\mathbf{x}}$ whose last $K$ elements match the last $K$ elements of $\mathbf{x}$ but whose first $\widetilde{J}$ elements contain the subset of $\left(x_{1}, \ldots, x_{J}\right)$ whose indices match those of the moment inequalities with t-ratios less than or equal to $\sqrt{\log n}$, i.e. those that are not far from binding. ${ }^{16}$ Step 4 uses a collection of iid normal draws, $\left\{\boldsymbol{\zeta}^{(r)}\right\}_{r=1}^{R}$, to approximate the large-sample distribution of $T_{n}$ under the null. The appropriate multiplications by $\Phi$ ensure that this approximation includes all moment equalities, but excludes any moment inequality judged to be far from binding in step 3. Finally, step 5 computes the p-value of the test by comparing the actual test statistic $T_{n}\left(\boldsymbol{\vartheta}_{0}\right)$ to the collection of simulated test statistics $\left\{T_{n}^{(r)}\left(\boldsymbol{\vartheta}_{0}\right)\right\}_{r=1}^{R}$ from step 4. We now detail our Bonferroni-based confidence interval for $\beta .^{17}$

Algorithm 3.2 (Bonferroni-based Confidence Interval for $\beta$ ). Inputs: significance levels $\left(\delta_{1}, \delta_{2}\right)$; iid dataset $\left\{\mathbf{w}_{i}\right\}_{i=1}^{n}$; simulations $\left\{\boldsymbol{\zeta}^{(r)}\right\}_{r=1}^{R} \sim$ iid $N_{J+K}(\mathbf{0}, \mathbf{I})$.

1. Construct a $\left(1-\delta_{1}\right) \times 100 \%$ joint confidence set $\mathcal{C}\left(\delta_{1}\right)$ for $\boldsymbol{\vartheta}=\left(\alpha_{0}, \alpha_{1}\right)^{\prime}$.

(i) Let $\Lambda_{N}=\left\{0, \frac{1}{N}, \frac{2}{N}, \ldots, \frac{N-2}{N}, \frac{N-1}{N}\right\}$ where $N>1$ is a natural number.

(ii) Set $\Delta_{N}=\left\{\left(\alpha_{0}, \alpha_{1}\right) \in\left(\Lambda_{N} \times \Lambda_{N}\right): \alpha_{0}+\alpha_{1}<1\right\}$.

(iii) For each $\boldsymbol{\vartheta} \in \Delta_{N}$ calculate $\widehat{p}(\boldsymbol{\vartheta})$ using Algorithm 3.1, holding $\left\{\boldsymbol{\zeta}^{(r)}\right\}_{r=1}^{R}$ fixed.

(iv) Set $\mathcal{C}\left(\delta_{1}\right)=\left\{\boldsymbol{\vartheta} \in \Delta_{N}: \widehat{p}(\boldsymbol{\vartheta}) \geq \delta_{1}\right\}$.

2. Construct a $\left(1-\delta_{1}\right) \times 100 \%$ confidence interval $\left[\underline{s}\left(\delta_{1}\right), \bar{s}\left(\delta_{1}\right)\right]$ for $s \equiv\left(1-\alpha_{0}-\alpha_{1}\right)$.

(i) Set $\underline{s}\left(\delta_{1}\right)=\min \left\{\left(1-\alpha_{0}-\alpha_{1}\right):\left(\alpha_{0}, \alpha_{1}\right) \in \mathcal{C}\left(\delta_{1}\right)\right\}$.

(ii) Set $\bar{s}\left(\delta_{1}\right)=\max \left\{\left(1-\alpha_{0}-\alpha_{1}\right):\left(\alpha_{0}, \alpha_{1}\right) \in \mathcal{C}\left(\delta_{1}\right)\right\}$.

3. Construct a $\left(1-\delta_{2}\right) \times 100 \%$ confidence interval $\left[\underline{\theta}_{1}\left(\delta_{2}\right), \bar{\theta}_{1}\left(\delta_{2}\right)\right]$ for $\theta_{1}$.

(i) Use the standard IV interval from a regression of $y$ on $T$ with instrument $z$.

4. Construct the $(1-\delta) \times 100 \%$ Bonferroni-based confidence interval $[\underline{\beta}(\delta), \bar{\beta}(\delta)]$ for $\beta$.

\footnotetext{
${ }^{16}$ Although this does not affect the results of the procedure, notice that Algorithm 3.1 carries out moment selection in a slightly different way from the steps given by Andrews and Soares (2010). In particular, before carrying out any further calculations, we subset the correlation matrix $\widehat{\Omega}$ and normal vectors $\zeta^{(r)}$ to remove elements corresponding to moment inequalities deemed far from binding. In contrast, Andrews and Soares (2010) carry along the full set of inequalities throughout, but add $+\infty$ to the appropriate elements when computing $T_{n}^{(r)}$ to ensure that only the moment inequalities that are not far from binding affect the results. Although it requires more notation to describe, sub-setting is substantially faster, as it avoids carrying out computations for inequalities that cannot affect the result.

${ }^{17}$ Code implementing this procedure is available at https://github.com/fditraglia/mbereg.
} 
(i) Let $\delta=\delta_{1}+\delta_{2}$.

(ii) Set $\underline{\beta}(\delta)=\min \left\{\underline{s}\left(\delta_{1}\right) \times \underline{\theta}_{1}\left(\delta_{2}\right), \bar{s}\left(\delta_{1}\right) \times \underline{\theta}_{1}\left(\delta_{2}\right)\right\}$.

(iii) Set $\bar{\beta}(\delta)=\max \left\{\underline{s}\left(\delta_{1}\right) \times \bar{\theta}_{1}\left(\delta_{2}\right), \bar{s}\left(\delta_{1}\right) \times \bar{\theta}_{1}\left(\delta_{2}\right)\right\}$.

Step 1 of Algorithm 3.2 constructs a $\left(1-\delta_{1}\right) \times 100 \%$ joint confidence set $\mathcal{C}\left(\delta_{1}\right)$ for $\boldsymbol{\vartheta}=\left(\alpha_{0}, \alpha_{1}\right)$ by inverting the GMS test from Algorithm 3.1 over a discretized parameter space $\Delta_{N}$. Because the parameter space for $\left(\alpha_{0}, \alpha_{1}\right)$ is bounded, this is computationally straightforward. Note that the same normal draws $\left\{\boldsymbol{\zeta}^{(r)}\right\}_{r=1}^{R}$ are used to test each null hypothesis contained in $\Delta_{N}$. Step 2 projects $\mathcal{C}\left(\delta_{1}\right)$ to yield a $\left(1-\delta_{1}\right) \times 100 \%$ confidence interval for $s \equiv\left(1-\alpha_{0}-\alpha_{1}\right)$, simply taking the maximum and minimum values of $s$ in the discrete set $\mathcal{C}\left(\delta_{1}\right)$. Step 3 constructs the usual IV confidence interval for the reduced form parameter $\theta_{1}$, and step 4 combines the results of steps $2-3$ with Bonferroni's inequality to yield a $\left(1-\delta_{1}-\delta_{2}\right) \times 100 \%$ confidence interval for $\beta$. For some discussion of alternatives to Algorithm 3.2, see Footnote 12. Notice that, by construction, the Bonferroni interval for $\beta$ excludes zero if and only if the confidence interval for $\theta_{1}$ from step 3 of Algorithm 3.2 excludes zero. Under mild regularity conditions, the confidence interval from Algorithm 3.2 is uniformly asymptotically valid.

Theorem 3.1. Let $\mathbf{w}_{1}, \ldots, \mathbf{w}_{n}$ be an iid collection of observations satisfying the conditions of Theorems 2.2 and 2.3, and let $z$ be a strong instrument. Then, under standard regularity conditions, the confidence interval for $\beta$ from Algorithm 3.2 has asymptotic coverage probability no less than $1-\left(\delta_{1}+\delta_{2}\right)$ as $R, N, n \rightarrow \infty$ uniformly over the parameter space.

Theorem 3.1 is effectively a corollary of Theorem 1 from Andrews and Soares (2010), which establishes the uniform asymptotic validity of the GMS test, and Lemma 3.1, which accounts for preliminary estimation of $\gamma$ and $\mathbf{q}$. Given iid observations $\mathbf{w}_{i}$, the only substantive condition required for Theorem 3.1 is the joint asymptotic normality of $\sqrt{n} \bar{m}_{n}\left(\boldsymbol{\vartheta}_{0}, \boldsymbol{\tau}_{0}\right)$ and $\sqrt{n} \bar{h}_{n}\left(\boldsymbol{\vartheta}_{0}, \boldsymbol{\tau}_{0}\right)$, where $\bar{h}_{n}$ denotes the sample analogues for the full set of auxiliary moment conditions $\left(h^{I}, h^{E}\right)$ defined in Section 3.4. For further discussion of the regularity conditions required for the GMS procedure, see Appendix A3 of Andrews and Soares (2010). For some discussion of the regularity conditions required for Lemma 3.1, see Section 3.4.

Theorem 3.1 invokes the higher-moment assumptions (Assumptions 2.5-2.6) under which we establish global identification of $\beta$ in Theorem 2.3, and Algorithm 3.1 likewise incorporates the higher-moment equality conditions that arise from this result. To proceed without these conditions, simply remove $m^{E}$ from the set of moment conditions used in the algorithm and leave the steps unchanged. In this case $\beta$ is no longer point identified but the inference procedure provides valid inference for the points in the sharp identified set from Theorem 2.2. 
Algorithm 3.2 can likewise be used in the case of an exogenous $T^{*}$, as in Mahajan (2006) and Frazis and Loewenstein (2003). As mentioned above in Section 3.1, the exogenous regressor case is subject to the same inferential difficulties as the endogenous case on which we focus in this paper. To accommodate an exogenous regressor, simply replace $m^{E}$ with the moment equalities described in Appendix C.

\subsection{Further Details Regarding Covariates}

The inference procedure described in the preceding sections holds $\mathbf{x}$ fixed, and is thus appropriate for examples with discrete covariates. To accommodate covariates more generally, there are several possible approaches. At one extreme, suppose one were willing to assume that $\left(\alpha_{0}, \alpha_{1}\right)$ did not vary with $\mathbf{x}$ and that $y=c+\beta T^{*}+\mathbf{x}^{\prime} \boldsymbol{\phi}+\varepsilon$, as in Frazis and Loewenstein (2003). In this case, the standard IV estimator identifies $\phi$ and one could simply augment the moment equalities $m^{E}$ from Equation 16 above to provide a preliminary estimator of $\phi$ in Algorithm 3.1. At the other extreme, if one wished to remain fully non-parametric, one could adopt the approach of Andrews and Shi (2014), based on kernel averaging near a fixed covariate value $\mathbf{x}=\mathbf{x}_{0}$. As a compromise between these two extremes, one could alternatively specify a semi-parametric model, perhaps along the lines of Section 4 of Lewbel (2007), and follow the approach of Andrews and Shi (2013). Both of these latter possibilities could be an interesting extension of the method described above.

\section{Simulation Study}

In this section we present results from a simulation study using the inference procedure described in Section 3.5 above. Unless otherwise specified, all calculations are based on 2000 simulation replications with $n=1000$ using Algorithm 3.2 with $R=5000$ simulation draws. Supplementary simulation results appear in Appendix D.

\subsection{Simulation DGP}

Our simulation design generates $n$ iid draws of the observables $\left(y_{i}, T_{i}, z_{i}\right)$ as follows:

1. Generate the instrumental variable $z$.

(i) For each $1 \leq i \leq n / 2$ set $z_{i}=0$.

(ii) For each $n / 2<i \leq n$, set $z_{i}=1$. 
2. Generate the error terms:

$$
\left[\begin{array}{l}
\eta_{i} \\
\varepsilon_{i}
\end{array}\right] \sim \operatorname{iid~N}\left(\left[\begin{array}{l}
0 \\
0
\end{array}\right],\left[\begin{array}{ll}
1 & \rho \\
\rho & 1
\end{array}\right]\right)
$$

3. Generate the unobserved regressor: $T_{i}^{*}=\mathbf{1}\left\{d_{0}+d_{1} z_{i}+\eta_{i}>0\right\}$.

4. Generate the outcome: $y_{i}=c+\beta T_{i}^{*}+\varepsilon_{i}$.

5. Generate the observed, mis-classified regressor $T$.

(i) For all $i$ with $T_{i}^{*}=0$ draw $T_{i} \sim \operatorname{iid} \operatorname{Bernoulli}\left(\alpha_{0}\right)$.

(ii) For all $i$ with $T_{i}^{*}=1$ draw $T_{i} \sim$ iid Bernoulli $\left(1-\alpha_{1}\right)$.

This DGP generates random variables that satisfy the conditions of Theorems 2.2 and 2.3. Thus $\beta$ is point identified, and all moment equalities and inequalities from Section 3 hold at the true parameter values of the DGP. Note from step 1 that we condition on the instrument $z$, holding it fixed in repeated samples. Our simulation varies the parameters $\left(\alpha_{0}, \alpha_{1}, \beta, n\right)$ over a grid. Because $\varepsilon$ has unit variance, values for $\beta$ are measured in standard deviations of the error. For simplicity we present results for $c=0, d_{0}=\Phi^{-1}(0.15)$, and $d_{1}=\Phi^{-1}(0.85)-$ $\Phi^{-1}(0.15)$, where $\Phi^{-1}(\cdot)$ denotes the quantile function of a standard normal random variable. Using these values for $\left(d_{0}, d_{1}\right)$ holds the unobserved first stage probabilities fixed: $p_{0}^{*}=0.15$ and $p_{1}^{*}=0.85$. In contrast the observed first-stage probabilities $p_{0}$ and $p_{1}$ vary with $\left(\alpha_{0}, \alpha_{1}\right)$ according to Lemma 2.1.

\subsection{Simulation Results}

As explained in Section 3.1 above, the just-identified, unconstrained GMM estimator based on Equation 10 suffers from weak identification and boundary value problems. Moreover, the estimator may not even exist in finite samples. Even when the GMM estimator exists, its asymptotic variance matrix could be numerically singular, so that the standard GMM confidence interval is undefined. Table 1 reports the percentage of simulation draws for which the standard GMM confidence interval is undefined, while Table 2 reports the coverage probability of a nominal 95\% GMM confidence interval, conditional on its existence.

We see from Table 1 that when $\beta$ is small compared to the error variance, the GMM confidence interval fails to exist with high probability. When $\beta=0.5$, for example, the interval is undefined approximately $30 \%$ of the time. As $\beta$ increases, however, it becomes less likely that the GMM interval is undefined. All else equal, larger amounts of mis-classification, i.e. higher values for $\left(\alpha_{0}, \alpha_{1}\right)$, increase the probability that the GMM interval fails to exist. 


\begin{tabular}{rc|rrrrrrrr}
\hline \hline & & & & \multicolumn{1}{|c}{$\beta$} & & & & \\
$\alpha_{0}$ & $\alpha_{1}$ & 0 & 0.25 & 0.5 & 0.75 & 1 & 1.5 & 2 & 3 \\
\hline 0.0 & 0.0 & 27 & 33 & 30 & 14 & 1 & 0 & 0 & 0 \\
& 0.1 & 27 & 32 & 29 & 13 & 2 & 0 & 0 & 0 \\
& 0.2 & 26 & 33 & 32 & 15 & 4 & 0 & 0 & 0 \\
& 0.3 & 26 & 34 & 30 & 17 & 5 & 0 & 0 & 0 \\
\hline 0.1 & 0.0 & 26 & 32 & 31 & 14 & 2 & 0 & 0 & 0 \\
& 0.1 & 26 & 36 & 32 & 16 & 4 & 0 & 0 & 0 \\
& 0.2 & 27 & 35 & 31 & 18 & 8 & 0 & 0 & 0 \\
& 0.3 & 25 & 35 & 32 & 21 & 11 & 1 & 0 & 0 \\
\hline 0.2 & 0.0 & 26 & 33 & 30 & 15 & 3 & 0 & 0 & 0 \\
& 0.1 & 26 & 33 & 30 & 19 & 6 & 0 & 0 & 0 \\
& 0.2 & 26 & 35 & 33 & 22 & 12 & 1 & 0 & 0 \\
& 0.3 & 26 & 35 & 33 & 26 & 15 & 3 & 0 & 0 \\
\hline 0.3 & 0.0 & 26 & 32 & 32 & 16 & 6 & 0 & 0 & 0 \\
& 0.1 & 24 & 35 & 33 & 21 & 11 & 1 & 0 & 0 \\
& 0.2 & 26 & 32 & 35 & 27 & 15 & 4 & 0 & 0 \\
& 0.3 & 26 & 35 & 35 & 28 & 21 & 7 & 2 & 0 \\
\hline
\end{tabular}

Table 1: Percentage of replications for which the standard GMM confidence interval based on Equation 10 fails to exist, either because the point estimate is $\mathrm{NaN}$ or the asymptotic covariance matrix is numerically singular. Calculations are based on 2000 replications of the DGP from 4.1 with $n=1000$.

\begin{tabular}{rr|rrrrrrrr}
\hline \hline & & & & \multicolumn{1}{|c}{$\beta$} & & & & \\
$\alpha_{0}$ & $\alpha_{1}$ & 0 & 0.25 & 0.5 & 0.75 & 1 & 1.5 & 2 & 3 \\
\hline 0.0 & 0.0 & 72 & 62 & 62 & 80 & 92 & 95 & 94 & 95 \\
& 0.1 & 72 & 62 & 63 & 79 & 92 & 95 & 96 & 95 \\
& 0.2 & 73 & 61 & 61 & 77 & 90 & 96 & 96 & 96 \\
& 0.3 & 73 & 59 & 62 & 76 & 88 & 95 & 96 & 95 \\
\hline 0.1 & 0.0 & 73 & 63 & 60 & 78 & 91 & 95 & 96 & 96 \\
& 0.1 & 73 & 58 & 59 & 77 & 90 & 95 & 95 & 94 \\
& 0.2 & 73 & 59 & 61 & 75 & 86 & 95 & 95 & 94 \\
& 0.3 & 74 & 59 & 58 & 71 & 82 & 94 & 96 & 96 \\
\hline 0.2 & 0.0 & 74 & 62 & 60 & 78 & 91 & 95 & 96 & 96 \\
& 0.1 & 73 & 60 & 61 & 74 & 87 & 95 & 96 & 94 \\
& 0.2 & 73 & 58 & 57 & 70 & 81 & 93 & 95 & 95 \\
& 0.3 & 73 & 58 & 56 & 66 & 78 & 92 & 95 & 96 \\
\hline 0.3 & 0.0 & 74 & 62 & 60 & 76 & 89 & 95 & 96 & 96 \\
& 0.1 & 75 & 59 & 58 & 71 & 82 & 93 & 96 & 95 \\
& 0.2 & 74 & 61 & 56 & 65 & 78 & 90 & 96 & 96 \\
& 0.3 & 73 & 58 & 55 & 64 & 71 & 88 & 93 & 96 \\
\hline
\end{tabular}

Table 2: Coverage (\%) of the standard nominal 95\% GMM confidence interval for $\beta$ based on Equation 10. Coverage is calculated only for those simulation draws for which the interval exists. (See Table 1.) Calculations are based on 2000 replications of the DGP from 4.1 with $n=1000$. 


\begin{tabular}{rr|rrrrrrrr}
\hline \hline & & \multicolumn{1}{|c}{$\beta$} \\
$\alpha_{0}$ & $\alpha_{1}$ & 0 & 0.25 & 0.5 & 0.75 & 1 & 1.5 & 2 & 3 \\
\hline 0.0 & 0.0 & 19.07 & 3.44 & 1.86 & 1.32 & 0.87 & 0.47 & 0.37 & 0.35 \\
& 0.1 & 17.52 & 3.47 & 1.92 & 1.41 & 1 & 0.61 & 0.51 & 0.46 \\
& 0.2 & 17.41 & 3.51 & 1.9 & 1.45 & 1.1 & 0.76 & 0.65 & 0.58 \\
& 0.3 & 18.23 & 3.34 & 1.92 & 1.48 & 1.24 & 0.91 & 0.79 & 0.7 \\
\hline 0.1 & 0.0 & 17.13 & 3.51 & 1.86 & 1.38 & 0.97 & 0.61 & 0.51 & 0.46 \\
& 0.1 & 17.88 & 3.33 & 1.85 & 1.45 & 1.13 & 0.78 & 0.67 & 0.6 \\
& 0.2 & 17.37 & 3.36 & 1.95 & 1.54 & 1.24 & 0.97 & 0.85 & 0.75 \\
& 0.3 & 18.07 & 3.33 & 1.98 & 1.63 & 1.41 & 1.17 & 1.04 & 0.92 \\
\hline 0.2 & 0.0 & 17.79 & 3.39 & 1.92 & 1.45 & 1.11 & 0.75 & 0.65 & 0.58 \\
& 0.1 & 18.98 & 3.43 & 1.96 & 1.54 & 1.26 & 0.97 & 0.84 & 0.75 \\
& 0.2 & 18.25 & 3.26 & 1.92 & 1.64 & 1.45 & 1.2 & 1.06 & 0.95 \\
& 0.3 & 19.03 & 3.31 & 2.02 & 1.75 & 1.66 & 1.49 & 1.33 & 1.19 \\
\hline 0.3 & 0.0 & 18.27 & 3.48 & 1.87 & 1.5 & 1.25 & 0.9 & 0.79 & 0.7 \\
& 0.1 & 19.4 & 3.41 & 1.96 & 1.63 & 1.43 & 1.18 & 1.04 & 0.92 \\
& 0.2 & 18.22 & 3.56 & 1.96 & 1.74 & 1.67 & 1.49 & 1.35 & 1.19 \\
& 0.3 & 17.56 & 3.55 & 2.13 & 1.96 & 1.86 & 1.86 & 1.74 & 1.55 \\
\hline
\end{tabular}

Table 3: Median width of the standard nominal 95\% GMM confidence interval for $\beta$ based on Equation 10. Coverage is calculated only for those simulation draws for which the interval exists. Calculations are based on 2000 replications of the DGP from 4.1 with $n=1000$.

Turning our attention to the simulation draws for which it is well-defined, we see from Tables 2 and 3 that the GMM confidence interval performs extremely poorly when $\beta$ is small. Substantial size distortions persist until $\beta$ is 1.5 or larger. All else equal, the size distortions are more severe the larger the amount of mis-classification error. For sufficiently large $\beta$, however, standard GMM inference performs well. As $\beta$ grows, the weak identification problem vanishes. For large enough $\beta$ the inference problem in effect becomes standard.

We now examine the performance of the Bonferroni-based confidence interval from Algorithm 3.2, beginning with its first step: a joint GMS confidence set for $\left(\alpha_{0}, \alpha_{1}\right)$. Table 4 presents coverage probabilities for a nominal 97.5\% GMS confidence set for $\left(\alpha_{0}, \alpha_{1}\right)$. Because these results are extremely fast to compute, Table 4 is based on 10,000 simulation replications. Aside from some slight under-coverage at intermediate values of $\left(\alpha_{0}, \alpha_{1}\right)$ when $\beta=3$, the GMS interval makes good on its promise of uniformly valid inference. As shown in Appendix D, the under-coverage problem appears to be a finite-sample artifact: if we increase $n$ to 2000 , the maximum size distortion becomes negligible. The GMS test tends, however, to be fairly conservative, particularly for larger values of $\left(\alpha_{0}, \alpha_{1}\right)$. When there is no mis-classification error, the GMS confidence sets are very nearly exact. Results for nominal 95\% and 90\% intervals are qualitatively similar: see Appendix D.

We now present results for the Bonferroni interval from Algorithm 3.2, setting $\delta_{1}=\delta_{2}=$ 


\begin{tabular}{rr|rrrrrrrr}
\hline \hline & & \multicolumn{7}{|c}{$\beta$} \\
$\alpha_{0}$ & $\alpha_{1}$ & 0 & 0.25 & 0.5 & 0.75 & 1 & 1.5 & 2 & 3 \\
\hline 0.0 & 0.0 & 97.7 & 97.7 & 97.6 & 97.7 & 98.0 & 98.0 & 97.4 & 97.9 \\
& 0.1 & 98.0 & 98.7 & 98.8 & 99.1 & 98.8 & 98.4 & 97.1 & 96.4 \\
& 0.2 & 98.4 & 98.5 & 98.9 & 98.9 & 98.8 & 98.6 & 98.0 & 97.0 \\
& 0.3 & 98.5 & 98.8 & 98.8 & 99.0 & 98.7 & 98.4 & 97.8 & 97.5 \\
\hline 0.1 & 0.0 & 98.1 & 98.5 & 98.3 & 98.8 & 98.8 & 98.4 & 96.8 & 95.7 \\
& 0.1 & 98.6 & 99.1 & 99.5 & 99.6 & 99.6 & 98.8 & 97.7 & 95.2 \\
& 0.2 & 99.0 & 99.3 & 99.7 & 99.8 & 99.7 & 98.9 & 97.5 & 95.7 \\
& 0.3 & 99.4 & 99.7 & 99.8 & 99.8 & 99.6 & 99.0 & 98.2 & 96.7 \\
\hline 0.2 & 0.0 & 98.6 & 98.5 & 98.6 & 98.9 & 98.7 & 98.2 & 97.7 & 97.0 \\
& 0.1 & 99.0 & 99.5 & 99.7 & 99.7 & 99.4 & 99.0 & 98.1 & 96.5 \\
& 0.2 & 99.5 & 99.7 & 99.8 & 99.7 & 99.4 & 99.0 & 97.8 & 96.8 \\
& 0.3 & 99.7 & 99.8 & 99.8 & 99.8 & 99.5 & 99.0 & 98.7 & 97.7 \\
\hline 0.3 & 0.0 & 98.7 & 98.7 & 98.8 & 98.7 & 98.7 & 98.2 & 98.1 & 97.6 \\
& 0.1 & 99.4 & 99.6 & 99.6 & 99.7 & 99.4 & 98.9 & 98.3 & 96.8 \\
& 0.2 & 99.8 & 99.8 & 99.7 & 99.8 & 99.5 & 99.1 & 98.5 & 97.8 \\
& 0.3 & 100.0 & 99.9 & 99.9 & 99.8 & 99.6 & 99.5 & 99.1 & 98.8 \\
\hline
\end{tabular}

Table 4: Coverage probability (1 - size) in percentage points of a 97.5\% GMS joint test for $\alpha_{0}$ and $\alpha_{1}$ using Algorithm 3.1 with $n=1000$. Calculations are based on 10,000 replications of the DGP from Section 4.1.

0.025 to yield an interval with asymptotic coverage no less that 95\%. ${ }^{18}$ Table 5 presents coverage probabilities in percentage points and Table 5 presents median widths.

The Bonferroni interval achieves its stated minimum coverage uniformly over the parameter space. When there is no mis-classification, $\alpha_{0}=\alpha_{1}$, its actual coverage is close or equal to $95 \%$. In the presence of mis-classification, however, the interval can be quite conservative, particularly for larger values of $\beta$. For smaller but nonzero values of $\beta$, this conservatism reflects the fact that the model is effectively partially identified: although Theorem 2.3 shows that $\left(\alpha_{0}, \alpha_{1}\right)$ are point identified for any $\beta \neq 0$, the amount of data required to distinguish one pair of alphas from another when $\beta$ is small would be astronomical.

In spite of its conservatism, the Bonferroni interval is informative, as we see from the median widths in Table 6 . Because median widths provide only a limited picture of the behavior of a confidence interval, Figures 1-3 present further evidence in the form of coverage functions ( 1 - power) for $\beta=0.5,1,3$. Coverage curves for additional values of $\beta$ and $n$ appear in Appendix D. Each figure holds the true value of $\beta$ fixed and varies $\left(\alpha_{0}, \alpha_{1}\right)$ over the grid $\{0,0.1,0.2\} \times\{0,0.2,0.2\}$. The plots within each Figure give coverage in percentage points as a function of the specified alternative for $\beta$. Solid curves are computed using the full

\footnotetext{
${ }^{18}$ In principle, one could optimize the choice of $\delta_{1}$ subject to the constraint $\delta_{1}+\delta_{2}=0.95$ to reduce the width of the resulting interval. In our experiments, there was no choice of $\delta_{1}$ that uniformly dominated for all values of $\left(\alpha_{0}, \alpha_{1}, \beta\right)$ so we report only results for $\delta_{1}=\delta_{2}$ here.
} 


\begin{tabular}{rr|rrrrrrrr}
\hline \hline & & \multicolumn{8}{|c}{$\beta$} \\
$\alpha_{0}$ & $\alpha_{1}$ & 0 & 0.25 & 0.5 & 0.75 & 1 & 1.5 & 2 & 3 \\
\hline 0.0 & 0.0 & 96 & 97 & 97 & 96 & 97 & 97 & 95 & 96 \\
& 0.1 & 97 & 99 & 99 & 99 & 99 & 100 & 100 & 99 \\
& 0.2 & 98 & 99 & 99 & 100 & 100 & 100 & 100 & 100 \\
& 0.3 & 97 & 100 & 100 & 100 & 100 & 100 & 100 & 100 \\
\hline 0.1 & 0.0 & 97 & 99 & 99 & 99 & 100 & 100 & 100 & 98 \\
& 0.1 & 98 & 100 & 100 & 100 & 100 & 100 & 100 & 100 \\
& 0.2 & 98 & 100 & 100 & 100 & 100 & 100 & 100 & 100 \\
& 0.3 & 97 & 100 & 100 & 100 & 100 & 100 & 100 & 100 \\
\hline 0.2 & 0.0 & 97 & 99 & 99 & 100 & 100 & 100 & 100 & 100 \\
& 0.1 & 98 & 100 & 100 & 100 & 100 & 100 & 100 & 100 \\
& 0.2 & 98 & 100 & 100 & 100 & 100 & 100 & 100 & 100 \\
& 0.3 & 98 & 100 & 100 & 100 & 100 & 100 & 100 & 100 \\
\hline 0.3 & 0.0 & 97 & 99 & 100 & 100 & 100 & 100 & 100 & 100 \\
& 0.1 & 97 & 100 & 100 & 100 & 100 & 100 & 100 & 100 \\
& 0.2 & 98 & 100 & 100 & 100 & 100 & 100 & 100 & 100 \\
& 0.3 & 98 & 100 & 100 & 100 & 100 & 100 & 100 & 100 \\
\hline
\end{tabular}

Table 5: Coverage probability in percentage points of a nominal $>95 \%$ Bonferroni confidence interval for $\beta$ using Algorithm 3.2 with $n=1000, R=5000$ and $\delta_{1}=\delta_{2}=0.025$. Calculations are based on 2000 replications of the DGP from Section 4.1.

\begin{tabular}{rr|rrrrrrrr}
\hline \hline & & \multicolumn{1}{|c}{$\beta$} \\
$\alpha_{0}$ & $\alpha_{1}$ & 0 & 0.25 & 0.5 & 0.75 & 1 & 1.5 & 2 & 3 \\
\hline 0.0 & 0.0 & 0.4 & 0.41 & 0.43 & 0.43 & 0.43 & 0.42 & 0.41 & 0.41 \\
& 0.1 & 0.45 & 0.47 & 0.54 & 0.59 & 0.63 & 0.7 & 0.75 & 0.86 \\
& 0.2 & 0.51 & 0.54 & 0.65 & 0.76 & 0.85 & 0.95 & 1.01 & 1.17 \\
& 0.3 & 0.58 & 0.62 & 0.79 & 0.95 & 1.07 & 1.17 & 1.24 & 1.48 \\
\hline 0.1 & 0.0 & 0.45 & 0.47 & 0.54 & 0.59 & 0.63 & 0.7 & 0.76 & 0.88 \\
& 0.1 & 0.51 & 0.54 & 0.66 & 0.77 & 0.86 & 1.03 & 1.18 & 1.46 \\
& 0.2 & 0.58 & 0.63 & 0.8 & 0.98 & 1.12 & 1.38 & 1.55 & 1.88 \\
& 0.3 & 0.67 & 0.75 & 1 & 1.25 & 1.46 & 1.74 & 1.94 & 2.4 \\
\hline 0.2 & 0.0 & 0.51 & 0.54 & 0.65 & 0.76 & 0.86 & 0.96 & 1.02 & 1.19 \\
& 0.1 & 0.58 & 0.63 & 0.81 & 0.99 & 1.14 & 1.42 & 1.64 & 2.08 \\
& 0.2 & 0.67 & 0.75 & 1.01 & 1.29 & 1.54 & 1.97 & 2.33 & 2.9 \\
& 0.3 & 0.81 & 0.91 & 1.3 & 1.7 & 2.09 & 2.73 & 3.13 & 3.9 \\
\hline 0.3 & 0.0 & 0.58 & 0.62 & 0.8 & 0.95 & 1.09 & 1.18 & 1.25 & 1.5 \\
& 0.1 & 0.68 & 0.74 & 1.01 & 1.26 & 1.49 & 1.84 & 2.13 & 2.78 \\
& 0.2 & 0.81 & 0.91 & 1.3 & 1.7 & 2.11 & 2.8 & 3.4 & 4.48 \\
& 0.3 & 1.01 & 1.16 & 1.74 & 2.35 & 2.93 & 4.17 & 5.2 & 6.85 \\
\hline
\end{tabular}

Table 6: Median width of a nominal $>95 \%$ Bonferroni confidence interval for $\beta$ using Algorithm 3.2 with $n=1000, R=5000$ and $\delta_{1}=\delta_{2}=0.025$. Calculations are based on 2000 replications of the DGP from Section 4.1. 
set of inequality moment conditions from Section 3.3, while dashed curves use only $m_{1}^{I}$, i.e. they do not impose the restrictions implied by non-differential measurement error. In each figure, the dashed horizontal line gives the nominal coverage probability, $95 \%$, while the dashed vertical lines are the reduced from and instrumental variables estimands: for $\beta \geq 0$ the reduced form is always smaller than the IV.

As seen from Figures 1-3, and their counterparts in Appendix D, the Bonferroni procedure has power against the alternative $\beta=0$, even when the true value of $\beta$ is small. As described in Section 3.5, the Bonferroni interval excludes zero if and only if the confidence interval for $\theta_{1}$ from which it is constructed also excludes zero. These figures also indicate the gains from including $m_{2}^{I}$, the moment inequalities that emerge from assuming non-differential measurement error: substantial increases in power against alternatives between the true parameter value and zero, particularly for larger values of $\beta$. Note moreover that the excellent performance of Bonferroni in the zero mis-classification case $\left(\alpha_{0}, \alpha_{1}\right)$ depends crucially on imposing the assumption of non-differential measurement error. As the true value of $\beta$ increases, the Bonferroni interval begins to have power against both the reduced form and IV estimands.

A drawback of the identification-robust inference procedure from Algorithm 3.2 becomes apparent when both $\beta$ and the mis-classification probabilities are large. In this case the confidence interval for $\beta$ is excessively wide, as we see from Table 6 and Figure $3 .{ }^{19}$ Note from Tables 1 and 2, that this is a region of the parameter space in which the plain-vanilla GMM confidence interval yields valid inference. Moreover, we see from Table 3 that the median width of the GMM interval is far more reasonable when $\beta$ is large, even in the presence of large amounts of mis-classification. It is important to stress that the source of this excess width is not the Bonferroni correction: the same behavior emerges if one projects a joint GMS confidence set for $\left(\alpha_{0}, \alpha_{1}, \beta\right)$ to yield marginal inference for $\beta$. Rather, it is the inevitable cost of applying a robust inference procedure in a region of the parameter space where standard inference performs well. While a detailed theoretical investigation of this problem is beyond the scope of the present paper, we now explore the performance of a "hybrid" confidence interval that uses a simple heuristic to transition between robust and standard inference. ${ }^{20}$ The procedure for constructing the hybrid interval is as follows. First compute the robust confidence interval based on Algorithm 3.2. Next, determine whether the GMM interval is well-defined: if so, determine whether it is contained within the robust interval. If the GMM interval exists and lies within the robust interval, report GMM; otherwise report the robust interval. Table 7 presents coverage probabilities (in percentage

\footnotetext{
${ }^{19}$ As expected, median widths decrease with sample size: see the results for $n=2000$ in Appendix D.

${ }^{20}$ This idea is related to Andrews (2016), although somewhat different in its details.
} 


\begin{tabular}{rr|rrrrrrrr}
\hline \hline & & \multicolumn{1}{|c}{$\beta$} & & & & & & \\
$\alpha_{0}$ & $\alpha_{1}$ & 0 & 0.25 & 0.5 & 0.75 & 1 & 1.5 & 2 & 3 \\
\hline 0.0 & 0.0 & 96 & 97 & 97 & 96 & 97 & 97 & 95 & 93 \\
& 0.1 & 97 & 99 & 99 & 99 & 99 & 98 & 96 & 95 \\
& 0.2 & 98 & 99 & 99 & 100 & 100 & 97 & 96 & 96 \\
& 0.3 & 97 & 100 & 100 & 100 & 99 & 96 & 96 & 96 \\
\hline 0.1 & 0.0 & 97 & 99 & 99 & 99 & 100 & 98 & 97 & 95 \\
& 0.1 & 98 & 100 & 100 & 100 & 100 & 96 & 96 & 96 \\
& 0.2 & 98 & 100 & 100 & 100 & 99 & 96 & 96 & 95 \\
& 0.3 & 97 & 100 & 100 & 100 & 97 & 95 & 96 & 96 \\
\hline 0.2 & 0.0 & 97 & 99 & 99 & 100 & 100 & 96 & 96 & 96 \\
& 0.1 & 98 & 100 & 100 & 100 & 99 & 96 & 96 & 96 \\
& 0.2 & 98 & 100 & 100 & 100 & 96 & 95 & 95 & 96 \\
& 0.3 & 98 & 100 & 100 & 98 & 95 & 95 & 95 & 96 \\
\hline 0.3 & 0.0 & 97 & 99 & 100 & 100 & 100 & 95 & 96 & 97 \\
& 0.1 & 97 & 100 & 100 & 100 & 97 & 94 & 96 & 96 \\
& 0.2 & 98 & 100 & 100 & 98 & 94 & 94 & 96 & 96 \\
& 0.3 & 98 & 100 & 99 & 96 & 92 & 94 & 95 & 96 \\
\hline
\end{tabular}

Table 7: Coverage probabilities (\%) of a hybrid confidence interval constructed from the nominal 95\% standard GMM interval and the $>95 \%$ Bonferroni confidence interval for $\beta$ using Algorithm 3.2 with $n=1000, R=5000$ and $\delta_{1}=\delta_{2}=0.025$. The hybrid interval reports Bonferroni unless the GMM interval exists and is contained within the Bonferroni interval. Calculations are based on 2000 replications of the DGP from Section 4.1.

points) and Table 8 median widths for the resulting hybrid confidence interval. Coverage plots for $\beta=1,2,3$ appear in Figures 4-6. Plots for additional values of $\beta$ and $n$ appear in Appendix D. The conventions of these figures are identical to those of Figures 1-3 with one exception: in Figures 4-6 the dashed curves correspond to the hybrid confidence interval.

The hybrid interval performs extremely well: with the exception of a slight size distortion at $\left(\alpha_{0}=\alpha_{1}=0.3, \beta=1\right)$ and $\left(\alpha_{0}=\alpha_{1}=0, \beta=3\right)$, it is effectively a free lunch. ${ }^{21}$ Note in particular that the coverage curves for the hybrid interval from Figures 4-6 (dashed curves) lie uniformly below those of the Bonferroni interval (solid curves) while still maintaining correct coverage at the true value of $\beta$. It could be interesting to investigate this idea further in future work.

\footnotetext{
${ }^{21}$ The distortion at $\left(\alpha_{0}=\alpha_{1}=0.3, \beta=1\right)$ disappears when $n$ increases to 2000: see Appendix D.
} 


\begin{tabular}{rr|rrrrrrrr}
\hline \hline & & \multicolumn{1}{|c}{$\beta$} \\
$\alpha_{0}$ & $\alpha_{1}$ & 0 & 0.25 & 0.5 & 0.75 & 1 & 1.5 & 2 & 3 \\
\hline 0.0 & 0.0 & 0.4 & 0.41 & 0.43 & 0.43 & 0.43 & 0.42 & 0.4 & 0.35 \\
& 0.1 & 0.45 & 0.47 & 0.54 & 0.59 & 0.63 & 0.67 & 0.52 & 0.46 \\
& 0.2 & 0.51 & 0.54 & 0.65 & 0.76 & 0.84 & 0.82 & 0.65 & 0.58 \\
& 0.3 & 0.58 & 0.62 & 0.79 & 0.95 & 1.05 & 0.96 & 0.79 & 0.7 \\
\hline 0.1 & 0.0 & 0.45 & 0.47 & 0.54 & 0.59 & 0.63 & 0.67 & 0.51 & 0.46 \\
& 0.1 & 0.51 & 0.54 & 0.66 & 0.77 & 0.86 & 0.92 & 0.69 & 0.61 \\
& 0.2 & 0.58 & 0.63 & 0.8 & 0.97 & 1.11 & 1.17 & 0.87 & 0.75 \\
& 0.3 & 0.67 & 0.75 & 1 & 1.25 & 1.4 & 1.4 & 1.06 & 0.92 \\
\hline 0.2 & 0.0 & 0.51 & 0.54 & 0.65 & 0.76 & 0.85 & 0.83 & 0.65 & 0.58 \\
& 0.1 & 0.58 & 0.63 & 0.81 & 0.99 & 1.12 & 1.18 & 0.86 & 0.75 \\
& 0.2 & 0.67 & 0.75 & 1.01 & 1.29 & 1.48 & 1.56 & 1.08 & 0.95 \\
& 0.3 & 0.81 & 0.91 & 1.3 & 1.67 & 1.95 & 1.77 & 1.35 & 1.2 \\
\hline 0.3 & 0.0 & 0.58 & 0.62 & 0.8 & 0.95 & 1.07 & 0.95 & 0.8 & 0.7 \\
& 0.1 & 0.68 & 0.74 & 1.01 & 1.26 & 1.43 & 1.48 & 1.06 & 0.93 \\
& 0.2 & 0.81 & 0.91 & 1.3 & 1.66 & 1.98 & 1.94 & 1.37 & 1.19 \\
& 0.3 & 1.01 & 1.16 & 1.73 & 2.24 & 2.71 & 2.33 & 1.78 & 1.55 \\
\hline
\end{tabular}

Table 8: Median width of a hybrid confidence interval constructed from the nominal $95 \%$ standard GMM interval and the $>95 \%$ Bonferroni confidence interval for $\beta$ using Algorithm 3.2 with $n=$ $1000, R=5000$ and $\delta_{1}=\delta_{2}=0.025$. The hybrid interval reports Bonferroni unless the GMM interval exists and is contained within the Bonferroni interval. Calculations are based on 2000 replications of the DGP from Section 4.1. 

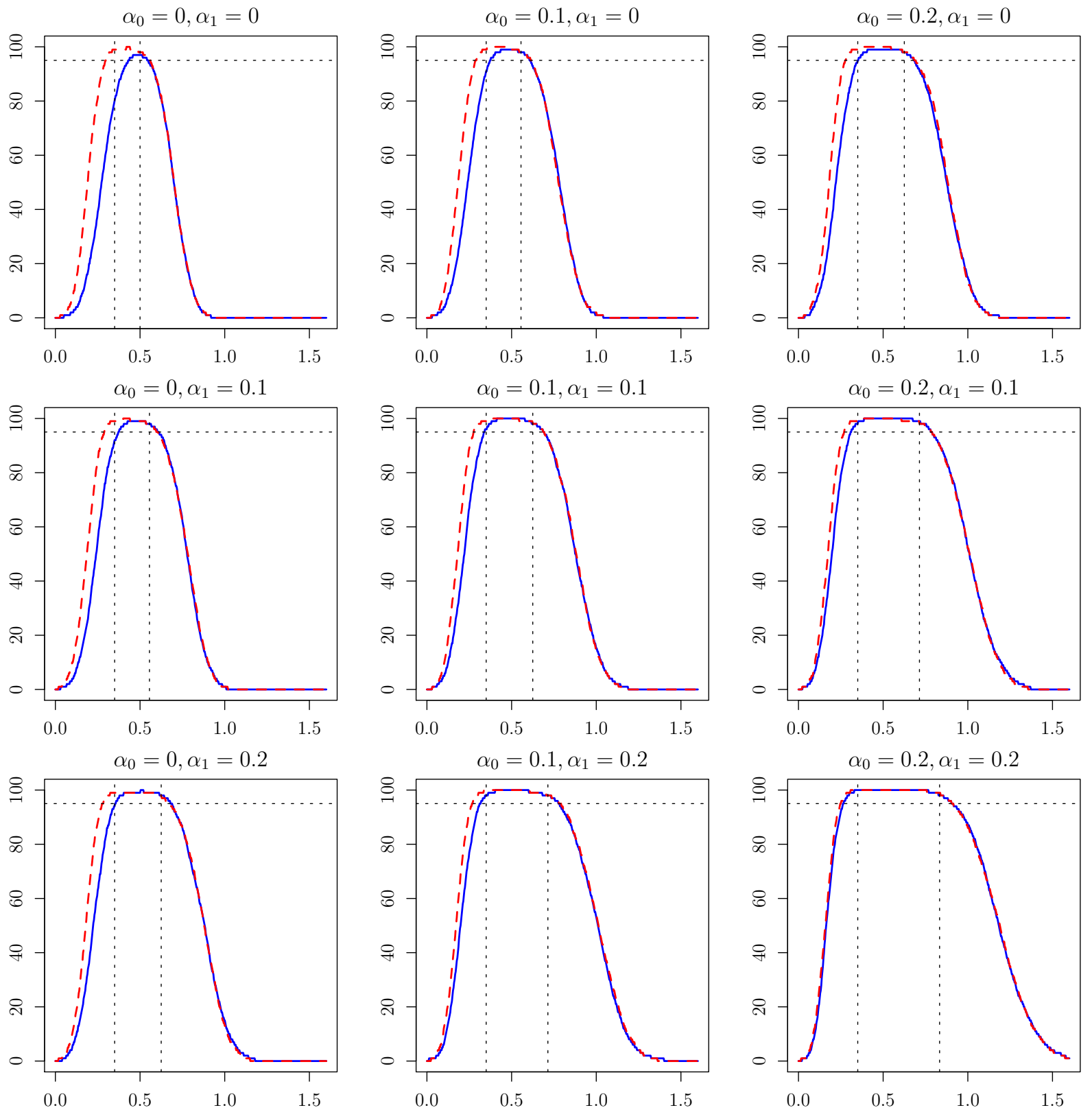

Figure 1: Coverage curves (1 - power) for $\beta$ when the truth is $\beta=0.5$, from a nominal $>95 \%$ Bonferroni confidence interval using Algorithm 3.2, with $n=1000$ and $R=5000$. The solid curve uses all moment inequalities from Section 3.3 in the GMS step, while the dashed curve excludes $m_{2}^{I}$, those implied by non-differential measurement error. The dashed horizontal line gives the nominal coverage (95\%), while dashed vertical lines are the reduced form estimand (left) and the IV estimand (right). Calculations are based on 2000 replications of the DGP from Section 4.1. 

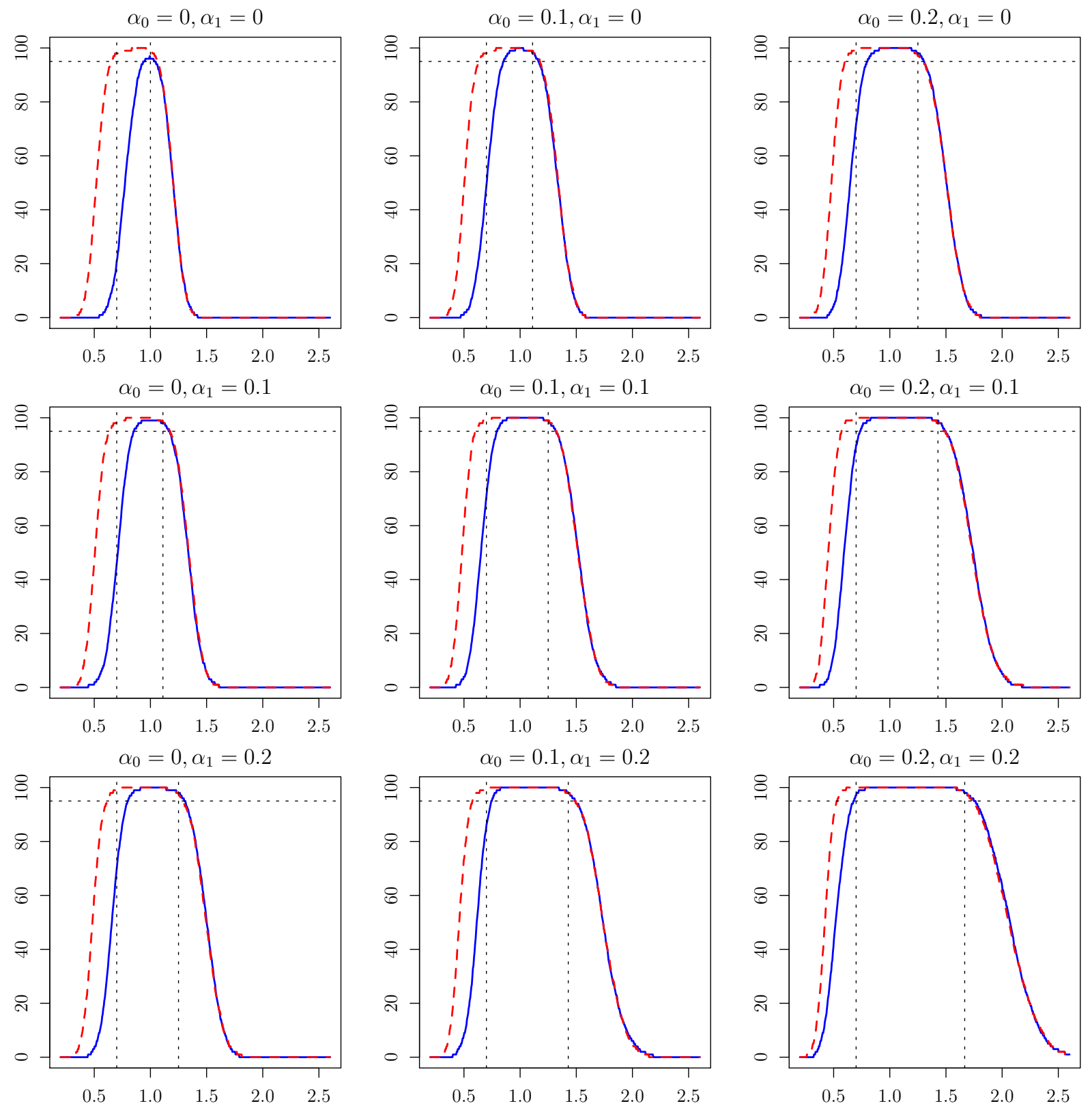

Figure 2: Coverage curves (1 - power) for $\beta$ when the truth is $\beta=1$, from a nominal $>95 \%$ Bonferroni confidence interval using Algorithm 3.2, with $n=1000$ and $R=5000$. The solid curve uses all moment inequalities from Section 3.3 in the GMS step, while the dashed curve excludes $m_{2}^{I}$, those implied by non-differential measurement error. The dashed horizontal line gives the nominal coverage (95\%), while dashed vertical lines are the reduced form estimand (left) and the IV estimand (right). Calculations are based on 2000 replications of the DGP from Section 4.1. 

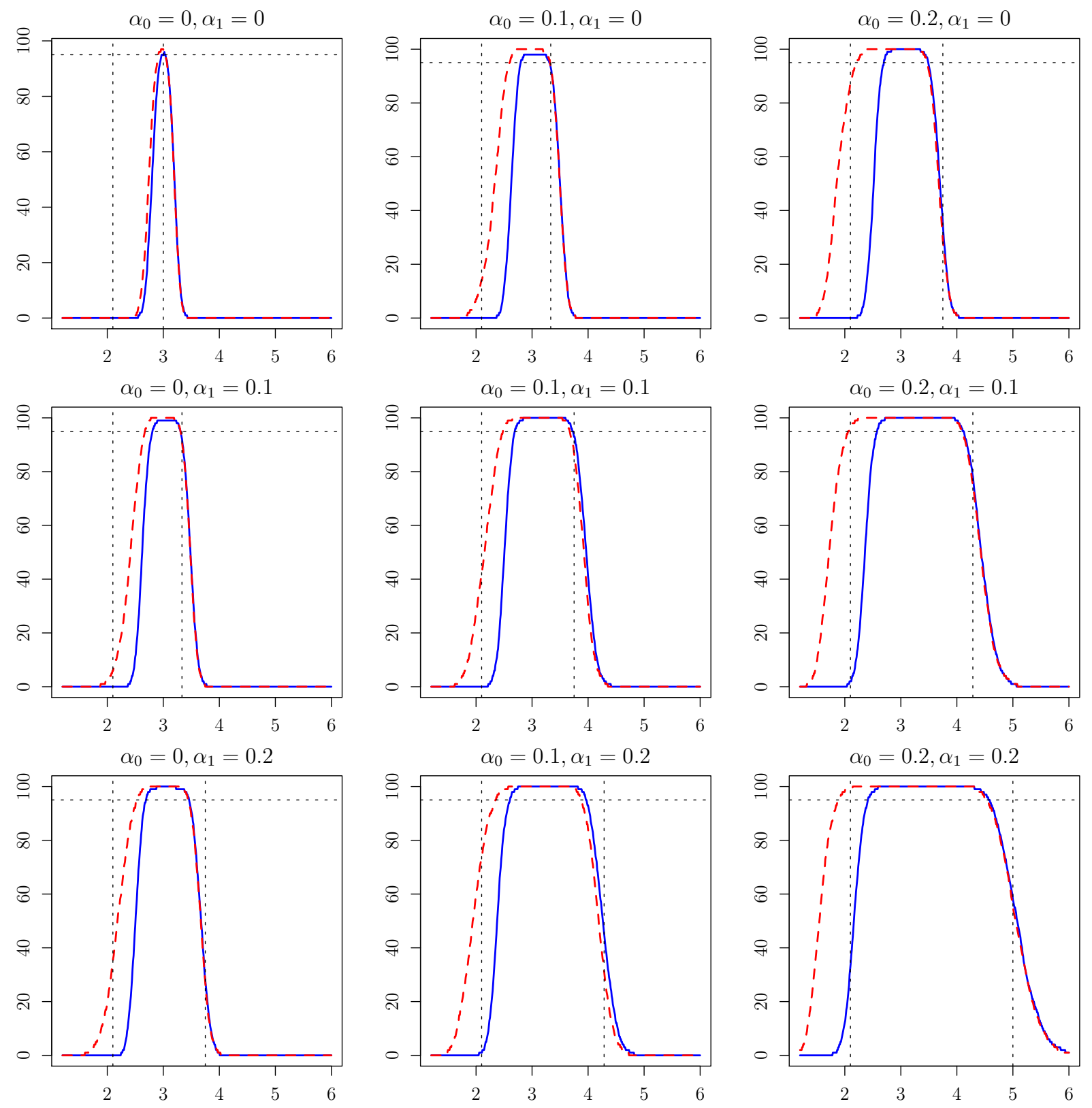

Figure 3: Coverage curves (1 - power) for $\beta$ when the truth is $\beta=3$, from a nominal $>95 \%$ Bonferroni confidence interval using Algorithm 3.2, with $n=1000$ and $R=5000$. The solid curve uses all moment inequalities from Section 3.3 in the GMS step, while the dashed curve excludes $m_{2}^{I}$, those implied by non-differential measurement error. The dashed horizontal line gives the nominal coverage (95\%), while dashed vertical lines are the reduced form estimand (left) and the IV estimand (right). Calculations are based on 2000 replications of the DGP from Section 4.1. 

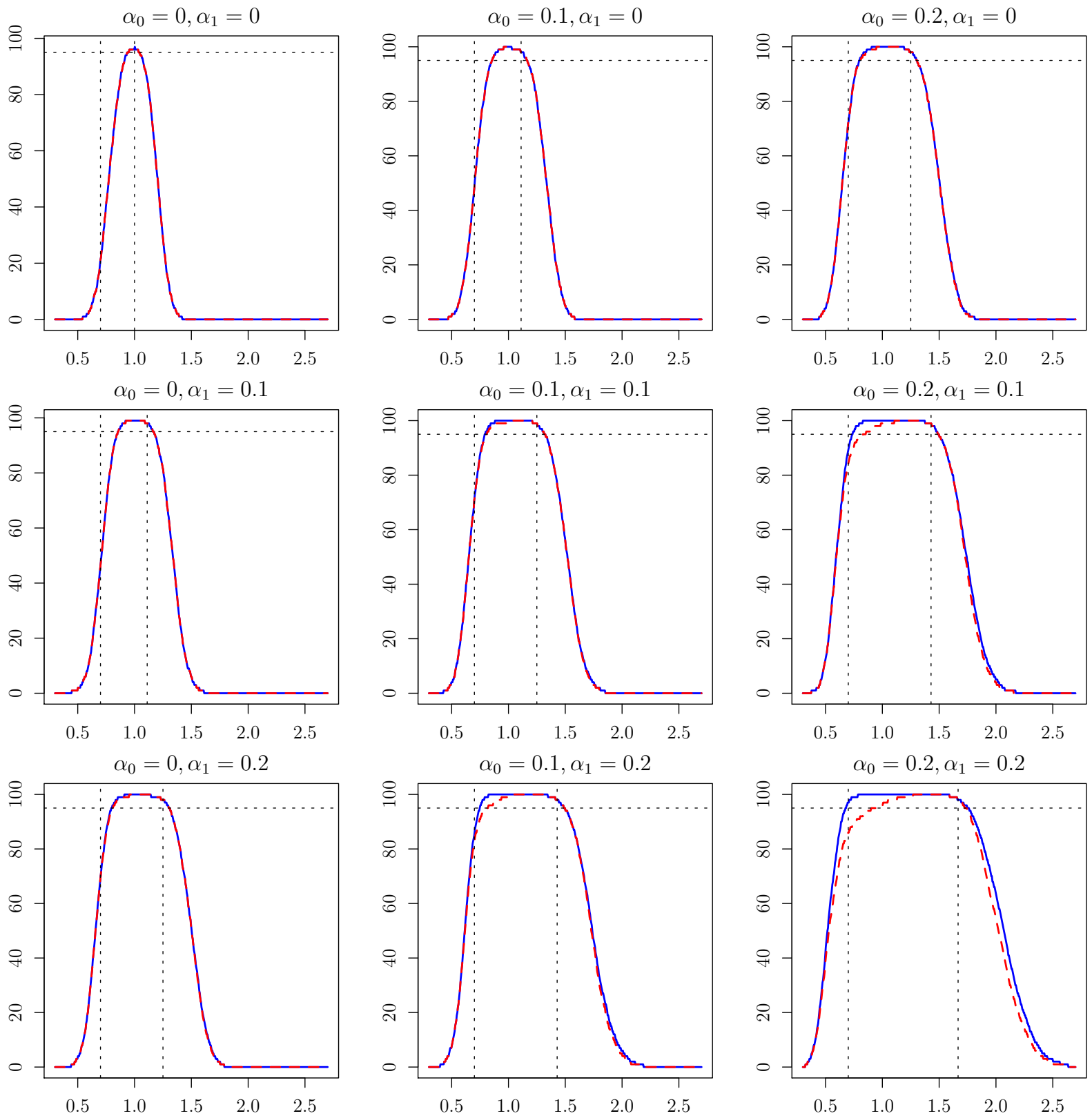

Figure 4: Comparison of Coverage curves (1 - power) for $\beta$ when the truth is $\beta=1$ : the solid curve corresponds the Bonferroni nominal > 95\% interval from Algorithm 3.2 and the dashed curve to the hybrid interval from Tables 7-8. The dashed horizontal line gives the nominal coverage (95\%), while dashed vertical lines are the reduced form estimand (left) and the IV estimand (right). Results are based on 2000 simulation replications from the DGP in Section 4.1 with $n=1000$. 

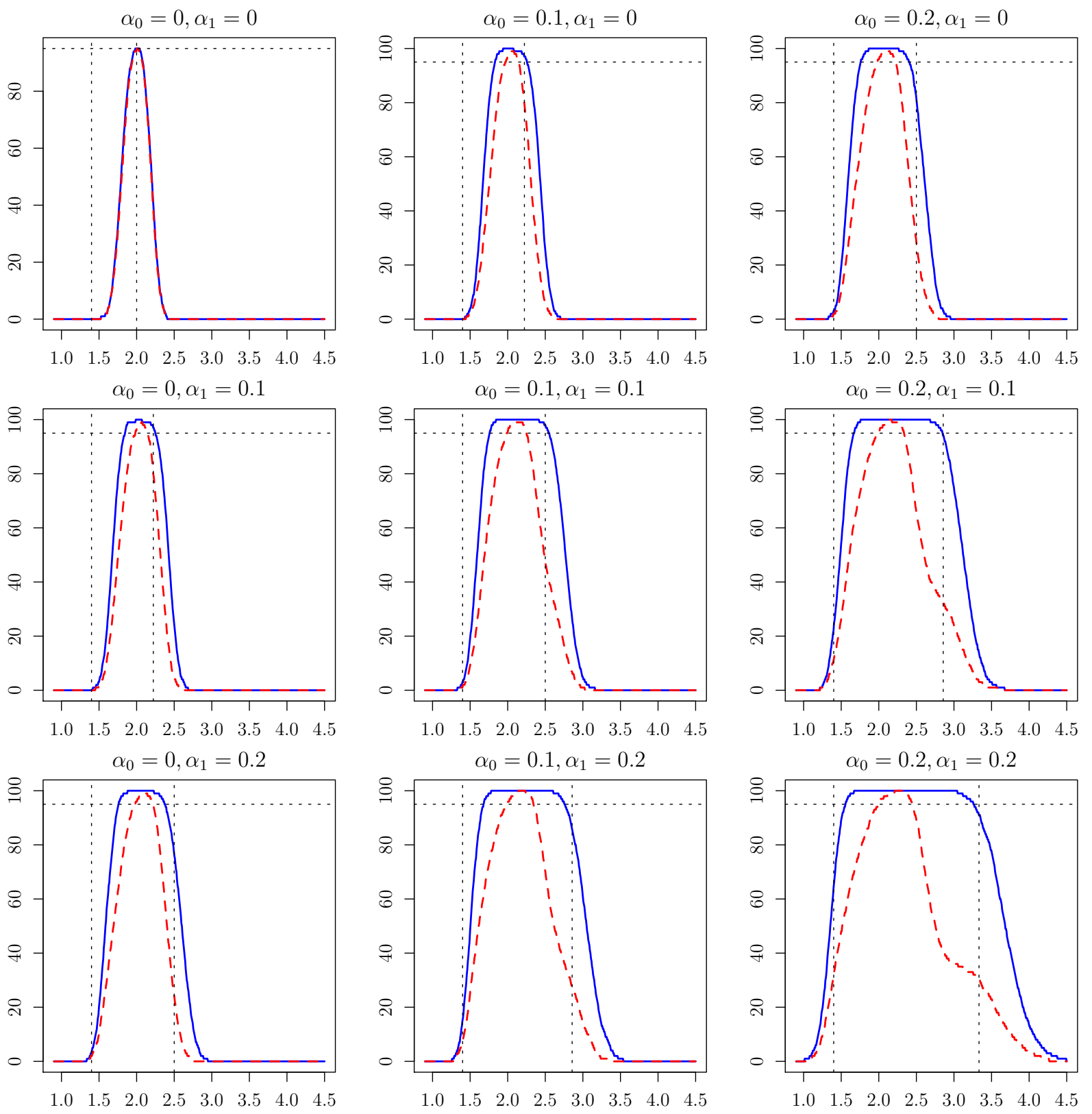

Figure 5: Comparison of Coverage curves (1 - power) for $\beta$ when the truth is $\beta=2$ : the solid curve corresponds the Bonferroni nominal > 95\% interval from Algorithm 3.2 and the dashed curve to the hybrid interval from Tables 7-8. The dashed horizontal line gives the nominal coverage (95\%), while dashed vertical lines are the reduced form estimand (left) and the IV estimand (right). Results are based on 2000 simulation replications from the DGP in Section 4.1 with $n=1000$. 

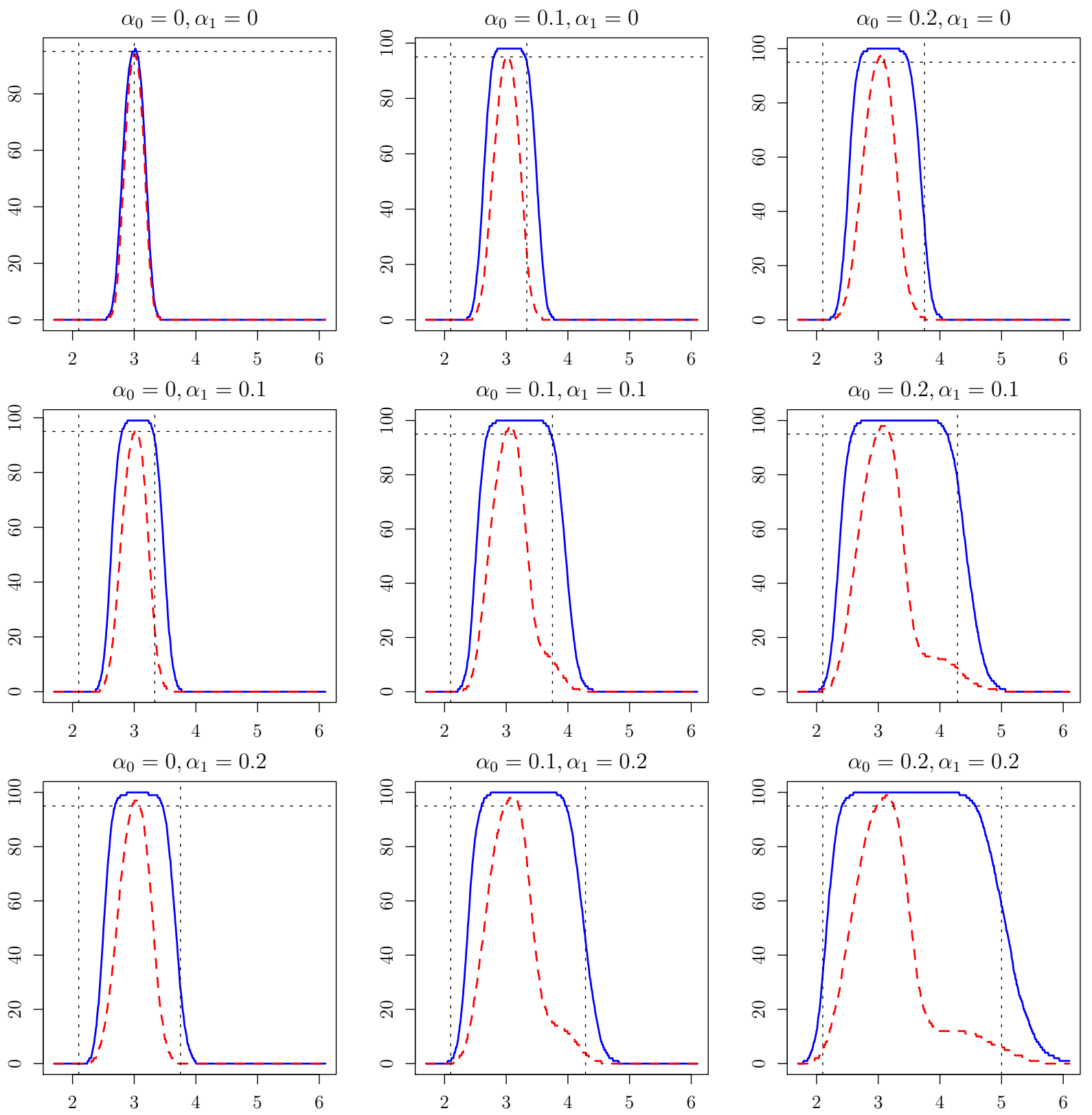

Figure 6: Comparison of Coverage curves ( 1 - power) for $\beta$ when the truth is $\beta=3$ : the solid curve corresponds the Bonferroni nominal > 95\% interval from Algorithm 3.2 and the dashed curve to the hybrid interval from Tables 7-8. The dashed horizontal line gives the nominal coverage (95\%), while dashed vertical lines are the reduced form estimand (left) and the IV estimand (right). Results are based on 2000 simulation replications from the DGP in Section 4.1 with $n=1000$. 


\section{Conclusion}

This paper has studied identification and inference for a mis-classified, binary, endogenous regressor in an additively separable model using a discrete instrumental variable. We have shown that the only existing identification result for this model is incorrect, and gone on to derive the sharp identified set under standard first-moment assumptions from the literature. Strengthening these assumptions to hold for second and third moments, we have established point identification for the effect of interest. Inference in models with mis-classification error is complicated by problems of weak identification and parameters on the boundary. To address these challenges, we have proposed a Bonferroni-based procedure for identification robust inference, using both the moment equalities from our identification results and moment inequalities from our partial identification results. This procedure is computationally attractive and performs well in simulations. An interesting extension of the results presented here would be to explore the more general case of a discrete endogenous regressor subject to mis-classification error, possibly by combining our approach with the matrix factorization techniques from $\mathrm{Hu}$ (2008). Another interesting extension, inspired by our hybrid confidence interval heuristic from Section 4, would be to study the transition between robust and standard inference in moment condition models. It may be possible, for example, to adapt the techniques of Andrews (2016) in this direction to provide similar theoretical guarantees.

\section{A Proofs}

Throughout the following arguments, we suppress dependence on $\mathbf{x}$ for simplicity.

\section{A.1 Partial Identification Results}

Proof of Lemma 2.1. Follows from a simple calculation using the law of total probability.

Proof of Lemma 2.2. Immediate since $\operatorname{Cov}(z, T)=\left(1-\alpha_{0}-\alpha_{1}\right) \operatorname{Cov}\left(z, T^{*}\right)$ by Lemma 2.1 .

Proof of Theorem 2.1. We first show that so long as $\alpha_{0} \leq p_{k} \leq 1-\alpha_{1}$ then we can construct a valid joint probability distribution for $\left(T^{*}, T, z\right)$ that satisfies our assumptions. First decompose the joint probability mass function as

$$
p\left(T^{*}, T, z\right)=p\left(T \mid T^{*}, z\right) p\left(T^{*} \mid z\right) p(z) .
$$

By Assumption 2.2 (ii), $p\left(T \mid T^{*}, z\right)=p\left(T \mid T^{*}\right)$ and thus $\alpha_{0}$ and $\alpha_{1}$ fully determine $p\left(T \mid T^{*}, z\right)$. Under the proposed bounds, $\alpha_{0}$ and $\alpha_{1}$ are clearly valid probabilities. Since $p(z)$ is observed, it thus suffices to ensure that $p\left(T^{*} \mid z\right)$ is a valid probability mass function. By Lemma 2.1, $p_{k}^{*}=\left(p_{k}-\alpha_{0}\right) /\left(1-\alpha_{0}-\right.$ $\left.\alpha_{1}\right)$ and hence $0 \leq p_{k}^{*} \leq 1$ if and only if $\alpha_{0} \leq p_{k} \leq 1-\alpha_{1}$. Since $\left(p_{k}-p_{\ell}\right)=\left(p_{k}^{*}-p_{\ell}^{*}\right)\left(1-\alpha_{0}-\alpha_{1}\right)$, we have $p_{k}^{*} \neq p_{\ell}^{*}$ provided that $p_{k}-p_{\ell} \neq 0$ 
We now show how to construct a valid conditional distribution for $y$ given $\left(T^{*}, T, z\right)$ that satisfies our assumptions if $\beta\left(p_{k}-\alpha_{0}\right)=\left(1-\alpha_{0}-\alpha_{1}\right)[\mathbb{E}(y \mid z=k)-c]$ for all $k$. Define

$$
\begin{aligned}
r_{t k} & \equiv \mathbb{P}\left(T^{*}=1 \mid T=t, z=k\right) & F_{t}(\tau) & \equiv \mathbb{P}(y \leq \tau \mid z=k) \\
F_{t k}(\tau) & \equiv \mathbb{P}(y \leq \tau \mid T=t, z=k) & F_{t k}^{t^{*}}(\tau) & \equiv \mathbb{P}\left(y \leq \tau \mid T^{*}=t^{*}, T=t, z=k\right) \\
G_{k}(\tau) & \equiv \mathbb{P}(\varepsilon \leq \tau \mid z=k) & G_{t k}^{t^{*}}(\tau) & \equiv \mathbb{P}\left(\varepsilon \leq \tau \mid T^{*}=t^{*}, T=t, z=k\right) .
\end{aligned}
$$

Assumption 2.1 (i) implies a relationship between $G_{t k}^{t^{*}}$ and $F_{t k}^{t^{*}}$ for each $t^{*}$, namely

$$
G_{t k}^{0}(\tau)=F_{t k}^{0}(\tau+c), \quad G_{t k}^{1}(\tau)=F_{t k}^{1}(\tau+c+\beta)
$$

and thus we see that

$$
\begin{aligned}
G_{k}(\tau)= & r_{1 k} p_{k} F_{1 k}^{1}(\tau+c+\beta)+r_{0 k}\left(1-p_{k}\right) F_{0 k}^{1}(\tau+c+\beta) \\
& +\left(1-r_{1 k}\right) p_{k} F_{1 k}^{0}(\tau+c)+\left(1-r_{0 k}\right)\left(1-p_{k}\right) F_{0 k}^{0}(\tau+c)
\end{aligned}
$$

applying the law of total probability and Bayes' rule. Moreover, again applying the law of total probability,

$$
F_{t k}(\tau)=r_{t k} F_{t k}^{1}(\tau)+\left(1-r_{t k}\right) F_{t k}^{0}(\tau)
$$

for all $t, k \in\{0,1\}$, and by Bayes' rule,

$$
r_{1 k}=\frac{\left(1-\alpha_{1}\right) p_{k}^{*}}{p_{k}}, \quad r_{0 k}=\frac{\alpha_{1} p_{k}^{*}}{1-p_{k}} .
$$

There are four cases, corresponding to different possibilities for the $r_{t k}$.

Case I: $r_{1 k}=0, r_{0 k} \neq 0 \quad$ By Equation A.4, this requires $\alpha_{1}=1$ which is ruled out by Assumption 2.2 (ii).

Case II: $r_{0 k}=r_{1 k}=0 \quad$ By Equation A.4, this requires $p_{k}^{*}=0$ which in turn requires $p_{k}=\alpha_{0}$. Moreover, by Equation A.3 we have $F_{t k}^{0}=F_{t k}$, while $F_{t k}^{1}$ is undefined. Substituting into Equation A.2,

$$
G_{k}(\tau)=p_{k} F_{1 k}(\tau+c)+\left(1-p_{k}\right) F_{0 k}(\tau+c)=F_{k}(\tau+c)
$$

Now, since $F_{k}(\tau+c)$ is the conditional CDF of $y-c$ given that $z=k$, and $G_{k}$ is the conditional $\mathrm{CDF}$ of $\varepsilon$ given $z=k$, we see that Assumption 2.1 (i) is satisfied if and only if $\mathbb{E}(y \mid z=k)=c$. But since $p_{k}=\alpha_{0}$ in this case, $c=c+\beta\left(p_{k}-\alpha_{0}\right) /\left(1-\alpha_{0}-\alpha_{1}\right)$.

Case III: $r_{1 k} \neq 0, r_{0 k}=0 \quad$ By Equation A. 4 this requires $\alpha_{1}=0$ and $p_{k}^{*} \neq 0$. By Equation A.3 we have $F_{0 k}^{0}=F_{0 k}$ and since $r_{1 k} \neq 1$, we can solve to obtain

$$
F_{1 k}^{1}(\tau)=\frac{1}{r_{1 k}}\left[F_{1 k}(\tau)-\left(1-r_{1 k}\right) F_{1 k}^{0}(\tau)\right]
$$

Substituting into Equation A.2, we obtain

$$
\begin{aligned}
G_{k}(\tau)= & {\left[\left(1-p_{k}\right) F_{0 k}(\tau+c)+p_{k} F_{1 k}(\tau+c+\beta)\right] } \\
& +p_{k}\left(1-r_{1 k}\right)\left[F_{1 k}^{0}(\tau+c)-F_{1 k}^{0}(\tau+c+\beta)\right]
\end{aligned}
$$


Now, $F_{0 k}(\tau+c)$ is the conditional $\mathrm{CDF}$ of $(y-c)$ given $(T=0, z=k)$ while $F_{1 k}(\tau+c+\beta)$ is the conditional CDF of $(y-c-\beta)$ given $(T=1, z=k)$. Similarly, $F_{1 k}^{0}(\tau+c)$ is the conditional CDF of $\varepsilon$ given $\left(T^{*}=0, T=1, z=k\right)$ while $F_{1 k}^{0}(\tau+c+\beta)$ is the conditional CDF of $(\varepsilon-\beta)$ given $\left(T^{*}=0, T=1, z=k\right)$. Since $G_{k}(\tau)$ is the conditional CDF of $\varepsilon$ given $z=k$, we see that Assumption 2.1 (iii) is satisfied if and only if

$$
\begin{aligned}
0= & \left(1-p_{k}\right) \mathbb{E}(y-c \mid T=0, z=k)+p_{k} \mathbb{E}(y-c-\beta \mid T=1, z=k) \\
& +p_{k}\left(1-r_{1 k}\right)\left[\mathbb{E}\left(\varepsilon \mid T^{*}=0, T=1, z=k\right)-\mathbb{E}\left(\varepsilon-\beta \mid T^{*}=0, T=1, z=k\right)\right]
\end{aligned}
$$

Rearranging, this is equivalent to

$$
\mathbb{E}(y \mid z=k)=c+\left(1-\alpha_{1}\right) \beta\left(\frac{p_{k}-\alpha_{0}}{1-\alpha_{0}-\alpha_{1}}\right)=c+\beta\left(\frac{p_{k}-\alpha_{0}}{1-\alpha_{0}-\alpha_{1}}\right)
$$

since $\alpha_{1}=0$ in this case. As explained above, $F_{0 k}^{0}=F_{0 k}$ in the present case while $F_{0 k}^{1}$ is undefined. We are free to choose any distributions for $F_{1 k}^{0}$ and $F_{1 k}^{1}$ that satisfy Equation A.3, for example $F_{1 k}^{0}=F_{1 k}^{1}=F_{1 k}$.

Case IV: $r_{1 k} \neq 0, r_{0 k} \neq 0 \quad$ In this case, we can solve Equation A.3 to obtain

$$
F_{t k}^{1}(\tau)=\frac{1}{r_{t k}}\left[F_{t k}(\tau)-\left(1-r_{t k}\right) F_{t k}^{0}(\tau)\right]
$$

Substituting this into Equation A.2, we have

$$
\begin{aligned}
G_{k}(\tau)=F_{k}(\tau+c+\beta) & +p_{k}\left(1-r_{1 k}\right)\left[F_{1 k}^{0}(\tau+c)-F_{1 k}^{0}(\tau+c+\beta)\right] \\
& +\left(1-p_{k}\right)\left(1-r_{0 k}\right)\left[F_{0 k}^{0}(\tau+c)-F_{0 k}^{0}(\tau+c+\beta)\right]
\end{aligned}
$$

using the fact that $F_{k}(\tau)=p_{k} F_{1 k}(\tau)+\left(1-p_{k}\right) F_{0 k}(\tau)$. Now, $F_{k}(\tau+c+\beta)$ is the conditional CDF of $(y-c-\beta)$ given $z=k$, while $F_{t k}^{0}(\tau+c)$ is the conditional CDF of $\varepsilon$ given $(T=t, z=k)$ and $F_{t k}^{0}(\tau+c+\beta)$ is the conditional $\mathrm{CDF}$ of $(\varepsilon-\beta)$ given $(T=t, z=k)$. Since $G_{k}(\tau)$ is the conditional CDF of $\varepsilon$ given $z=k$, we see that Assumption 2.1 (iii) is satisfied if and only if

$$
\begin{aligned}
0= & \mathbb{E}[y-c-\beta \mid z=k]+p_{k}\left(1-r_{1 k}\right)\left[\mathbb{E}\left(\varepsilon \mid T^{*}=0, T=1, z=k\right)-\mathbb{E}\left(\varepsilon-\beta \mid T^{*}=0, T=1, z=k\right)\right] \\
& +\left(1-p_{k}\right)\left(1-r_{0 k}\right)\left[\mathbb{E}\left(\varepsilon \mid T^{*}=0, T=0, z=k\right)-\mathbb{E}\left(\varepsilon-\beta \mid T^{*}=0, T=0, z=k\right)\right] \\
0= & \mathbb{E}[y-c-\beta \mid z=k]+\beta\left[p_{k}\left(1-r_{1 k}\right)+\left(1-p_{k}\right)\left(1-r_{0 k}\right)\right]
\end{aligned}
$$

But since $\left[p_{k}\left(1-r_{1 k}\right)+\left(1-p_{k}\right)\left(1-r_{0 k}\right)\right]=\left(1-p_{k}^{*}\right)$ and $p_{k}^{*}=\left(p_{k}-\alpha_{0}\right) /\left(1-\alpha_{0}-\alpha_{1}\right)$, this becomes

$$
\mathbb{E}[y \mid z=k]=c+\beta\left[\left(p_{k}-\alpha_{0}\right)\left(1-\alpha_{0}-\alpha_{1}\right)\right] .
$$

Thus, in this case we are free to choose any distributions for $F_{t k}^{0}$ and $F_{t k}^{1}$ that satisfy Equation A.3. For example we could take $F_{t k}^{0}=F_{t k}^{1}=F_{t k}$.

Proof of Corollary 2.1. Follows by plugging in the largest and smallest possible values for $\alpha_{0}+\alpha_{1}$ and taking the difference of the expressions for $\mathbb{E}[y \mid z=k]$

Proof of Theorem 2.2. Under Assumption 2.1 (i) and Assumption 2.2 (iii), we obtain $\mathbb{E}\left(y \mid T^{*}, T, z\right)=$ 
$\mathbb{E}\left(y \mid T^{*}, z\right)$. Hence, by iterated expectations

$$
\begin{aligned}
& \mathbb{E}(y \mid T=0, z=k)=\left(1-r_{0 k}\right) \mathbb{E}\left(y \mid T^{*}=0, z=k\right)+r_{0 k} \mathbb{E}\left(y \mid T^{*}=1, z=k\right) \\
& \mathbb{E}(y \mid T=1, z=k)=\left(1-r_{1 k}\right) \mathbb{E}\left(y \mid T^{*}=0, z=k\right)+r_{1 k} \mathbb{E}\left(y \mid T^{*}=1, z=k\right)
\end{aligned}
$$

where $r_{t k}$ is defined as in the proof of Theorem 2.1. This is system of two linear equations in two unknowns: $\mathbb{E}\left(y \mid T^{*}=0, z=k\right)$ and $\mathbb{E}\left(y \mid T^{*}=1, z=k\right)$. After some algebra, we find that the determinant is

$$
r_{1 k}-r_{0 k}=\left[\frac{p_{k}-\alpha_{0}}{1-\alpha_{0}-\alpha_{1}}\right]\left[\frac{1-p_{k}-\alpha_{1}}{p_{k}\left(1-p_{k}\right)}\right]
$$

and thus a unique solution exists provided that $\alpha_{0} \neq p_{k}$ and $\alpha_{1} \neq 1-p_{k}$. By our assumption that $\mathbb{E}[y \mid T=0, z=k] \neq \mathbb{E}[y \mid T=1, z=k]$, the system has no solution when the determinant condition fails. Thus, Assumption 2.2 (iii) rules out $\alpha_{0}=p_{k}$ and $\alpha_{1}=1-p_{k}$. Solving,

$$
\begin{aligned}
& \mu_{k}^{0} \equiv \mathbb{E}\left(y \mid T^{*}=0, z=k\right)=\left(\frac{1}{1-p_{k}-\alpha_{1}}\right)\left[\left(1-p_{k}\right) \mathbb{E}(y \mid T=0, z=k)-\alpha_{1} \mathbb{E}(y \mid z=k)\right] \\
& \mu_{k}^{1} \equiv \mathbb{E}\left(y \mid T^{*}=1, z=k\right)=\left(\frac{1}{p_{k}-\alpha_{0}}\right)\left[p_{k} \mathbb{E}(y \mid T=1, z=k)-\alpha_{0} \mathbb{E}(y \mid z=k)\right]
\end{aligned}
$$

Given $\left(\alpha_{0}, \alpha_{1}\right)$, we see that $r_{t k}, \mu_{k}^{0}$, and $\mu_{k}^{1}$ are fixed. The question is whether, for a given pair $\left(\alpha_{0}, \alpha_{1}\right)$ and observed CDFs $F_{t k}$, we can construct valid CDFs $F_{t k}^{0}, F_{t k}^{1}$ such that

$$
\int_{\mathbb{R}} \tau F_{t k}^{0}(d \tau)=\mu_{k}^{0}, \quad \int_{\mathbb{R}} \tau F_{t k}^{1}(d \tau)=\mu_{k}^{1}, \quad F_{t k}(\tau)=r_{t k} F_{t k}^{1}(\tau)+\left(1-r_{t k}\right) F_{t k}^{0}(\tau)
$$

where $F_{t k}$ and $F_{t k}^{t^{*}}$ are as defined in the proof of Theorem 2.2. For a given pair $(t, k)$, there are two cases: $0<r_{t k}<1$ and $r_{t k} \in\{0,1\}$.

Case I: $r_{t k} \in\{0,1\} \quad$ Suppose that $r_{t k}=1$. Then, $\mu_{k}^{1}=\mathbb{E}[y \mid T=t, z=k]$ so we can simply set $F_{t k}^{1}=F_{t k}$. In this case $F_{t k}^{0}$ is undefined. If instead $r_{t k}=0$, then $\mu_{k}^{0}=\mathbb{E}[y \mid T=t, z=k]$ so we can simply set $F_{t k}^{0}=F_{t k}$. In this case $F_{t k}^{1}$ is undefined.

Case II: $0<r_{t k}<1 \quad$ Define

$$
\begin{aligned}
\mu_{t k}(\xi) & =\mathbb{E}\left[y \mid y \in I_{t k}(\xi), T=t, z=k\right] \\
I_{t k}(\xi) & =\left[F_{t k}^{-1}\left(1-\xi-r_{t k}\right), F_{t k}^{-1}(1-\xi)\right]
\end{aligned}
$$

for $t, k=0,1$ where $0 \leq \xi \leq 1-r_{t k}$ and $F_{t k}^{-1}$ is the quantile function of $y$ given $(T=t, z=k)$. We see that $\mu_{t k}$ is a decreasing function of $\xi$ that attains its maximum at $\xi=0$ and minimum at $\xi=1-r_{t k}$. Define these extrema as $\underline{\mu}_{t k}=\mu_{t k}\left(1-r_{t k}\right)$ and $\bar{\mu}_{t k}=\mu_{t k}(0)$.

Suppose first that $\mu_{k}^{1}$ does not lie in the interval $\left[\underline{\mu}_{t k}, \bar{\mu}_{t k}\right]$. We show that it is impossible to construct valid CDFs $F_{t k}^{0}$ and $F_{t k}^{1}$ that satisfy $F_{t k}(\tau)=r_{t k} F_{t k}^{1}(\tau)+\left(1-r_{t k}\right) F_{t k}^{0}(\tau)$ where $F_{t k}$ and $F_{t k}^{t^{*}}$ are as defined in the proof of Theorem 2.2. Since $r_{t k} \neq 1$, we can solve the expression for $F_{t k}$ to yield $F_{t k}^{0}(\tau)=\left[F_{t k}(\tau)-r_{t k} F_{t k}^{1}(\tau)\right] /\left(1-r_{t k}\right)$. Hence, since $r_{t k} \neq 0$, the requirement that $0 \leq F_{t k}^{0}(\tau) \leq 1$ implies

$$
\frac{F_{t k}(\tau)-\left(1-r_{t k}\right)}{r_{t k}} \leq F_{t k}^{1}(\tau) \leq \frac{F_{t k}(\tau)}{r_{t k}}
$$


Now define

$$
\begin{aligned}
& \underline{F}_{t k}^{1}(\tau)=\min \left\{1, F_{t k}(\tau) / r_{t k}\right\} \\
& \bar{F}_{t k}^{1}(\tau)=\max \left\{0, F_{t k}(\tau) / r_{t k}-\left(1-r_{t k}\right) / r_{t k}\right\}
\end{aligned}
$$

Combining Equation A.5 with the requirement that $0 \leq F_{t k}^{1}(\tau) \leq 1$, we see that

$$
\bar{F}_{t k}^{1}(\tau) \leq F_{t k}^{1}(\tau) \leq \underline{F}_{t k}^{1}(\tau)
$$

Hence $\bar{F}_{t k}^{1}$ first-order stochastically dominates $F_{t k}^{1}$ which in turn first-order stochastically dominates $\underline{F}_{t k}^{1}$. It follows that

$$
\int \tau \underline{F}_{t k}^{1}(d \tau) \leq \int \tau F_{t k}^{1}(d \tau) \leq \int \tau \bar{F}_{t k}^{1}(d \tau)
$$

But notice that

$$
\underline{\mu}_{t k}=\int \tau \underline{F}_{t k}^{1}(d \tau), \quad \mu_{k}^{1}=\int \tau F_{t k}^{1}(d \tau), \quad \bar{\mu}_{t k}=\int \tau \bar{F}_{t k}^{1}(d \tau)
$$

so we have $\underline{\mu}_{t k} \leq \mu_{k}^{1} \leq \bar{\mu}_{t k}$ which contradicts $\mu_{k}^{1} \notin\left[\underline{\mu}_{t k}, \bar{\mu}_{t k}\right]$.

Now suppose that $\mu_{k}^{1} \in\left[\underline{\mu}_{t k}, \bar{\mu}_{t k}\right]$. Since $y$ is assumed to follow a continuous distribution conditional on $(T, z), \mu_{t k}$ is continuous on its domain and takes on all values in $\left[\underline{\mu}_{t k}, \bar{\mu}_{t k}\right]$ by the intermediate value theorem. Thus, there exists a $\xi^{*}$ such that $\mu_{t k}\left(\xi^{*}\right)=\mu_{k}^{1}$. Now let $f_{t k}(\tau)=$ $d F_{t k}(\tau) / d \tau$ which is non-negative by the assumption that $y$ is continuously distributed. Define the densities

$$
f_{t k}^{1}(\tau)=\frac{f_{t k}(\tau) \times \mathbf{1}\left\{\tau \in I_{t k}\left(\xi^{*}\right)\right\}}{r_{t k}}, \quad f_{t k}^{0}(\tau)=\frac{f_{t k}(\tau) \times \mathbf{1}\left\{\tau \in I_{t k}\left(\xi^{*}\right)\right\}}{1-r_{t k}} .
$$

Clearly $f_{t k}^{1} \geq 0$ and $f_{t k}^{0} \geq 0$. Integrating,

$$
\begin{aligned}
\int_{\mathbb{R}} f_{t k}^{1}(\tau) d \tau & =\frac{1}{r_{t k}} \int_{I_{t k}\left(\xi^{*}\right)} f_{t k}(\tau) d \tau=1 \\
\int_{\mathbb{R}} f_{t k}^{0}(\tau) d \tau & =\frac{1}{1-r_{t k}} \int_{I_{t k}^{C}\left(\xi^{*}\right)} f_{t k}(\tau) d \tau=1
\end{aligned}
$$

where $I_{t k}^{C}$ is the complement of $I_{t k}$. And, by construction

$$
r_{t k} \int_{A} f_{t k}^{1}(\tau) d \tau+\left(1-r_{t k}\right) \int_{A} f_{t k}^{0}(\tau) d \tau=\int_{A} f_{t k}(\tau) d \tau
$$

for any set $A$. Finally,

$$
\int_{\mathbb{R}} \tau f_{t k}^{1}(\tau) d \tau=\frac{1}{r_{t k}} \int_{I_{t k}\left(\xi^{*}\right)} \tau f_{t k}(\tau) d \tau=\mu_{t k}\left(\xi^{*}\right)=\mu_{k}^{1} .
$$

The result now follows by appealing to the proof of Theorem 2.1. 


\section{A.2 Point Identification Results}

In the proofs of Lemma 2.3, Lemma 2.4, and Theorem 2.3, we use the shorthand

$$
\pi \equiv \operatorname{Cov}(T, z), \quad \eta_{j} \equiv \operatorname{Cov}\left(y^{j}, z\right), \quad \tau_{j} \equiv \operatorname{Cov}\left(T y^{j}, z\right)
$$

for $j=1,2,3$. Using this notation, Lemma 2.2 becomes $\eta_{1}=\pi \theta_{1}$, while Lemma 2.3 becomes $\eta_{2}=2 \tau_{1} \theta_{1}-\pi \theta_{2}$, and Lemma 2.4 becomes $\eta_{3}=3 \tau_{2} \theta_{1}-3 \tau_{1} \theta_{2}+\pi \theta_{3}$.

Proof of Lemma 2.3. By Assumption 2.1 (i) and the basic properties of covariance,

$$
\begin{aligned}
& \eta_{2}=\beta^{2} \operatorname{Cov}\left(T^{*}, z\right)+2 \beta\left[c \operatorname{Cov}\left(T^{*}, z\right)+\operatorname{Cov}\left(T^{*} \varepsilon, z\right)\right]+2 c \operatorname{Cov}(\varepsilon, z)+\operatorname{Cov}\left(\varepsilon^{2}, z\right) \\
& \tau_{1}=c \pi+\operatorname{Cov}(T \varepsilon, z)+\beta \operatorname{Cov}\left(T T^{*}, z\right)
\end{aligned}
$$

using the fact that $T^{*}$ is binary. Now, by Assumptions 2.1 (iii) and 2.5 we have $\operatorname{Cov}(\varepsilon, z)=$ $\operatorname{Cov}\left(\varepsilon^{2}, z\right)=0$. And, using Assumptions 2.2 (i) and (ii), one can show that $\operatorname{Cov}\left(T T^{*}, z\right)=(1-$ $\left.\alpha_{1}\right) \operatorname{Cov}\left(T^{*}, z\right)$ and $\operatorname{Cov}\left(T^{*}, z\right)=\pi /\left(1-\alpha_{0}-\alpha_{1}\right)$. Hence,

$$
\begin{aligned}
\eta_{2} & =\theta_{1}(\beta+2 c) \pi+2 \beta \operatorname{Cov}\left(T^{*} \varepsilon, z\right) \\
2 \tau_{1} \theta_{1}-\pi \theta_{2} & =\left[2 \theta_{1} c+2 \theta_{1}^{2}\left(1-\alpha_{1}\right)-\theta_{2}\right] \pi+2 \theta_{1} \operatorname{Cov}(T \varepsilon, z)
\end{aligned}
$$

but since $\theta_{2}=\theta_{1}^{2}\left[\left(1-\alpha_{1}\right)+\alpha_{0}\right]$, we see that $\left[2 \theta_{1}^{2}\left(1-\alpha_{1}\right)-\theta_{2}\right]=\theta_{1} \beta$. Thus, it suffices to show that $\beta \operatorname{Cov}\left(T^{*} \varepsilon, z\right)=\theta_{1} \operatorname{Cov}(T \varepsilon, z)$. This equality is trivially satisfied when $\beta=0$, so suppose that $\beta \neq 0$. In this case it suffices to show that $\left(1-\alpha_{0}-\alpha_{1}\right) \operatorname{Cov}\left(T^{*} \varepsilon, z\right)=\operatorname{Cov}(T \varepsilon, z)$. Define $m_{t k}^{*}=\mathbb{E}\left[\varepsilon \mid T^{*}=t, z=k\right]$ and $p_{k}^{*}=\mathbb{P}\left(T^{*}=1 \mid z=k\right)$. Then, by iterated expectations, Bayes' rule, and Assumption 2.2 (iii)

$$
\begin{aligned}
\operatorname{Cov}\left(T^{*} \varepsilon, z\right) & =q(1-q)\left(p_{1}^{*} m_{11}^{*}-p_{0}^{*} m_{10}^{*}\right) \\
\operatorname{Cov}(T \varepsilon, z) & =q(1-q)\left\{\left(1-\alpha_{1}\right)\left[p_{1}^{*} m_{11}^{*}-p_{0}^{*} m_{10}^{*}\right]+\alpha_{0}\left[\left(1-p_{1}^{*}\right) m_{01}^{*}-\left(1-p_{0}^{*}\right) m_{00}^{*}\right]\right\}
\end{aligned}
$$

But by Assumption 2.1 (iii), $\mathbb{E}[\varepsilon \mid z=k]=m_{1 k}^{*} p_{k}^{*}+m_{0 k}^{*}\left(1-p_{k}^{*}\right)=0$ and thus we obtain $m_{0 k}^{*}\left(1-p_{k}^{*}\right)=$ $-m_{1 k}^{*} p_{k}^{*}$. Therefore $\left(1-\alpha_{0}-\alpha_{1}\right) \operatorname{Cov}\left(T^{*} \varepsilon, z\right)=\operatorname{Cov}(T \varepsilon, z)$ as required.

Proof of Lemma 2.4. Since $T^{*}$ is binary, if follows from the basic properties of covariance that,

$$
\begin{aligned}
& \eta_{3}=\operatorname{Cov}\left[(c+\varepsilon)^{3}, z\right]+3 \beta \operatorname{Cov}\left[(c+\varepsilon)^{2} T^{*}, z\right]+3 \beta^{2} \operatorname{Cov}\left[(c+\varepsilon) T^{*}, z\right]+\beta^{3} \operatorname{Cov}\left(T^{*}, z\right) \\
& \tau_{2}=\operatorname{Cov}\left[(c+\varepsilon)^{2} T, z\right]+2 \beta \operatorname{Cov}\left[(c+\varepsilon) T T^{*}, z\right]+\beta^{2} \operatorname{Cov}\left(T T^{*}, z\right)
\end{aligned}
$$

By Assumptions 2.1 (iii), 2.5, and 2.6 (ii) $\operatorname{Cov}\left[(c+\varepsilon)^{3}, z\right]=0$. Expanding,

$$
\begin{aligned}
& \eta_{3}=3 \beta \operatorname{Cov}\left(T^{*} \varepsilon^{2}, z\right)+\left(3 \beta^{2}+6 c \beta\right) \operatorname{Cov}\left(T^{*} \varepsilon, z\right)+\left(\beta^{3}+3 c \beta^{2}+3 c^{2} \beta\right) \operatorname{Cov}\left(T^{*}, z\right) \\
& \tau_{2}=c^{2} \operatorname{Cov}(T, z)+\beta(\beta+2 c) \operatorname{Cov}\left(T T^{*}, z\right)+\operatorname{Cov}\left(T \varepsilon^{2}, z\right)+2 c \operatorname{Cov}(T \varepsilon, z)+2 \beta \operatorname{Cov}\left(T T^{*} \varepsilon, z\right)
\end{aligned}
$$

Now, define $s_{t k}^{*}=\mathbb{E}\left[\varepsilon^{2} \mid T^{*}=t, z=k\right]$ and $p_{k}^{*}=\mathbb{P}\left(T^{*}=1 \mid z=k\right)$. By iterated expectations, Bayes' rule, and Assumption 2.6 (i),

$$
\begin{aligned}
\operatorname{Cov}\left(T^{*} \varepsilon^{2}, z\right) & =q(1-q)\left(p_{1}^{*} s_{11}^{*}-p_{0}^{*} s_{10}^{*}\right) \\
\operatorname{Cov}\left(T \varepsilon^{2}, z\right) & =q(1-q)\left\{\left(1-\alpha_{1}\right)\left[p_{1}^{*} s_{11}^{*}-p_{0}^{*} s_{10}^{*}\right]+\alpha_{0}\left[\left(1-p_{1}^{*}\right) s_{01}^{*}-\left(1-p_{0}^{*}\right) s_{00}^{*}\right]\right\}
\end{aligned}
$$


By Assumption 2.5, $\mathbb{E}\left[\varepsilon^{2} \mid z=1\right]=\mathbb{E}\left[\varepsilon^{2} \mid z=0\right]$ and thus, by iterated expectations we have $p_{1}^{*} s_{11}^{*}$ $p_{0}^{*} s_{10}^{*}=-\left[\left(1-p_{1}^{*}\right) s_{01}^{*}-\left(1-p_{0}^{*}\right) s_{00}^{*}\right]$ which implies

$$
\operatorname{Cov}\left(T \varepsilon^{2}, z\right)=\left(1-\alpha_{0}-\alpha_{1}\right) \operatorname{Cov}\left(T^{*} \varepsilon^{2}, z\right) .
$$

Similarly by iterated expectations and Assumptions 2.2 (i)-(ii)

$$
\operatorname{Cov}\left(T T^{*} \varepsilon, z\right)=q(1-q)\left(1-\alpha_{1}\right)\left(p_{1}^{*} m_{1 k}^{*}-p_{0}^{*} m_{10}^{*}\right)=\left(1-\alpha_{1}\right) \operatorname{Cov}\left(T^{*} \varepsilon, z\right)
$$

where $m_{t k}^{*}$ is defined as in the proof of Lemma 2.3. As shown in the proof of Lemma 2.3,

$$
\begin{aligned}
\operatorname{Cov}\left(T T^{*}, z\right) & =\left(1-\alpha_{1}\right) \operatorname{Cov}\left(T^{*}, z\right) \\
\operatorname{Cov}\left(T^{*}, z\right) & =\pi /\left(1-\alpha_{0}-\alpha_{1}\right) \\
\operatorname{Cov}\left(T^{*} \varepsilon, z\right) & =\operatorname{Cov}(T \varepsilon, z) /\left(1-\alpha_{0}-\alpha_{1}\right)
\end{aligned}
$$

and combining these equalities with Equations A.6 and A.7, it follows that

$$
\begin{gathered}
\tau_{2}=2\left[\left(1-\alpha_{1}\right)(c+\beta)-c \alpha_{0}\right] \operatorname{Cov}\left(T^{*} \varepsilon, z\right)+\left[\left(1-\alpha_{1}\right)(c+\beta)^{2}-c^{2} \alpha_{0}\right] \operatorname{Cov}\left(T^{*}, z\right) \\
+\left(1-\alpha_{0}-\alpha_{1}\right) \operatorname{Cov}\left(T^{*} \varepsilon^{2}, z\right) \\
\tau_{1}=\left(1-\alpha_{0}-\alpha_{1}\right) \operatorname{Cov}\left(T^{*} \varepsilon, z\right)+\left[\left(1-\alpha_{1}\right)(c+\beta)-c \alpha_{0}\right] \operatorname{Cov}\left(T^{*}, z\right)
\end{gathered}
$$

using $\tau_{1}=c \pi+\operatorname{Cov}(T \varepsilon, z)+\beta \operatorname{Cov}\left(T T^{*}, z\right)$ as shown in the proof of Lemma 2.3. Thus,

$$
3 \tau_{2} \theta_{1}-3 \tau_{1} \theta_{2}+\pi \theta_{3}=K_{1} \operatorname{Cov}\left(T^{*} \varepsilon^{2}, z\right)+K_{2} \operatorname{Cov}\left(T^{*} \varepsilon, z\right)+K_{3} \operatorname{Cov}\left(T^{*}, z\right)
$$

where $K_{1} \equiv 3 \theta_{1}\left(1-\alpha_{0}-\alpha_{1}\right)=3 \beta$ and

$$
\begin{aligned}
& K_{2} \equiv 6 \theta_{1}\left[\left(1-\alpha_{1}\right)(c+\beta)-c \alpha_{0}\right]-3 \theta_{2}\left(1-\alpha_{0}-\alpha_{1}\right) \\
& K_{3} \equiv 3 \theta_{1}\left[\left(1-\alpha_{1}\right)(c+\beta)^{2}-c^{2} \alpha_{0}\right]-3 \theta_{2}\left[\left(1-\alpha_{1}\right)(c+\beta)-c \alpha_{0}\right]+\theta_{3}\left(1-\alpha_{0}-\alpha_{1}\right)
\end{aligned}
$$

Substituting the definitions of $\theta_{1}, \theta_{2}$, and $\theta_{3}$ from Equations 6-8, tedious but straightforward algebra shows that $K_{2}=3 \beta^{2}+6 c \beta$ and $K_{3}=\beta^{3}+3 c \beta^{2}+3 c^{2} \beta$. Therefore the coefficients of $\eta_{3}$ equal those of $3 \tau_{2}-3 \tau_{1} \theta_{2}+\pi \theta_{3}$ and the result follows.

Proof of Theorem 2.3. Collecting the results of Lemmas 2.2-2.4, we have

$$
\eta_{1}=\pi \theta_{1}, \quad \eta_{2}=2 \tau_{1} \theta_{1}-\pi \theta_{2}, \quad \eta_{3}=3 \tau_{2} \theta_{1}-3 \tau_{1} \theta_{2}+\pi \theta_{3}
$$

which is a linear system in $\theta_{1}, \theta_{2}, \theta_{3}$ with determinant $-\pi^{3}$. Since $\pi \neq 0$ by assumption 2.1 (ii), $\theta_{1}, \theta_{2}$ and $\theta_{3}$ are identified. Now, so long as $\beta \neq 0$, we can rearrange Equations 7 and 8 to obtain

$$
\begin{aligned}
& A=\theta_{2} / \theta_{1}^{2}=1+\left(\alpha_{0}-\alpha_{1}\right) \\
& B=\theta_{3} / \theta_{1}^{3}=\left(1-\alpha_{0}-\alpha_{1}\right)^{2}+6 \alpha_{0}\left(1-\alpha_{1}\right)
\end{aligned}
$$

Equation A.8 gives $\left(1-\alpha_{1}\right)=A-\alpha_{0}$. Hence $\left(1-\alpha_{0}-\alpha_{1}\right)=A-2 \alpha_{0}$ and $\alpha_{0}\left(1-\alpha_{1}\right)=\alpha_{0}\left(A-\alpha_{0}\right)$. Substituting into Equation A.9 and simplifying, $\left(A^{2}-B\right)+2 A \alpha_{0}-2 \alpha_{0}^{2}=0$. Substituting for $\alpha_{0}$ analogously yields a quadratic in $\left(1-\alpha_{1}\right)$ with identical coefficients. It follows that one root of 
$\left(A^{2}-B\right)+2 A r-2 r^{2}=0$ is $\alpha_{0}$ and the other is $1-\alpha_{1}$. Solving,

$$
r=\frac{A}{2} \pm \sqrt{3 A^{2}-2 B}=\frac{1}{\theta_{1}^{2}}\left(\frac{\theta_{2}}{2} \pm \sqrt{3 \theta_{2}^{2}-2 \theta_{1} \theta_{3}}\right)
$$

By Equations 7 and 8,

$$
\begin{aligned}
3 \theta_{2}^{2}-2 \theta_{1} \theta_{3} & =3\left[\theta_{1}^{2}\left(1+\alpha_{0}-\alpha_{1}\right)\right]^{2}-2 \theta_{1}\left\{\theta_{1}^{3}\left[\left(1-\alpha_{0}-\alpha_{1}\right)^{2}+6 \alpha_{0}\left(1-\alpha_{1}\right)\right]\right\} \\
& =\theta_{1}^{4}\left\{3\left(1+\alpha_{0}-\alpha_{1}\right)^{2}-2\left[\left(1-\alpha_{0}-\alpha_{1}\right)^{2}+6 \alpha_{0}\left(1-\alpha_{1}\right)\right]\right\} .
\end{aligned}
$$

Expanding the first term we find that

$$
\begin{aligned}
3\left(1+\alpha_{0}-\alpha_{1}\right)^{2} & =3\left[1+2\left(\alpha_{0}-\alpha_{1}\right)+\left(\alpha_{0}-\alpha_{1}\right)^{2}\right] \\
& =3+6 \alpha_{0}-6 \alpha_{1}+3 \alpha_{0}^{2}+3 \alpha_{1}^{2}-6 \alpha_{0} \alpha_{1}
\end{aligned}
$$

and expanding the second

$$
\begin{aligned}
2\left[\left(1-\alpha_{0}-\alpha_{1}\right)^{2}+6 \alpha_{0}\left(1-\alpha_{1}\right)\right] & =2\left[1-2\left(\alpha_{0}+\alpha_{1}\right)+\left(\alpha_{0}+\alpha_{1}\right)^{2}+6 \alpha_{0}-6 \alpha_{0} \alpha_{1}\right] \\
& =2+8 \alpha_{0}-4 \alpha_{1}+2 \alpha_{0}^{2}+2 \alpha_{1}^{2}-8 \alpha_{0} \alpha_{1} .
\end{aligned}
$$

Therefore

$$
\begin{aligned}
3 \theta_{2}^{2}-2 \theta_{1} \theta_{3} & =\theta_{1}^{4}\left\{1-2 \alpha_{0}-2 \alpha_{1}+\alpha_{0}^{2}-\alpha_{1}^{2}+2 \alpha_{0} \alpha_{1}\right\} \\
& =\theta_{1}^{4}\left[\left(1-\alpha_{0}-\alpha_{1}\right)^{2}\right]
\end{aligned}
$$

which is strictly greater than zero since $\theta_{1} \neq 0$ and $\alpha_{0}+\alpha_{1} \neq 0$. It follows that both roots of the quadratic are real. Moreover, $3 \theta_{2}^{2} / \theta_{1}^{4}-2 \theta_{3} / \theta_{1}^{3}$ identifies $\left(1-\alpha_{0}-\alpha_{1}\right)^{2}$. Substituting into Equation 6 , it follows that $\beta$ is identified up to sign. If $\alpha_{0}+\alpha_{1}<1$ then $\operatorname{sign}(\beta)=\operatorname{sign}\left(\theta_{1}\right)$ so that both the sign and magnitude of $\beta$ are identified. If $\alpha_{0}+\alpha_{1}<1$ then $1-\alpha_{1}>\alpha_{0}$ so $\left(1-\alpha_{1}\right)$ is the larger root of $\left(A^{2}-B\right)+2 A r-2 r^{2}=0$ and $\alpha_{0}$ is the smaller root.

\section{B Comment on Mahajan (2006) A.2}

Expanding on our discussion from Section 2.2 above, we now show that Mahajan's identification argument for an endogenous regressor in an additively separable model (A.2) is incorrect. Unless otherwise indicated, all notation used below is as defined in Section 2.

The first step of Mahajan (2006) A.2 argues (correctly) that under Assumptions 2.1 and 2.2 (i)-(ii), knowledge of $\alpha_{0}(\mathbf{x})$ and $\alpha_{1}(\mathbf{x})$ is sufficient to identify $\beta(\mathbf{x})$. This step is equivalent to our Lemma 2.2 above. The second step appeals to Mahajan (2006) Theorem 1 to argue that $\alpha_{0}(\mathbf{x})$ and $\alpha_{1}(\mathbf{x})$ are indeed point identified. To understand the logic of this second step, we first re-state Mahajan (2006) Theorem 1 in our notation. As in Section 2 above, $T^{*}$ denotes an unobserved binary random variable, $z$ is a instrument, $T$ an observed binary surrogate for $T^{*}, y$ an outcome of interest, and $\mathbf{x}$ a vector covariates.

Assumption B.1 (Mahajan (2006) Theorem 1). Define $g\left(T^{*}, \mathbf{x}\right) \equiv \mathbb{E}\left[y \mid \mathbf{x}, T^{*}\right]$ and $v \equiv$ $y-g\left(T^{*}, \mathbf{x}\right)$. Suppose that knowledge of $\left(y, T^{*}, \mathbf{x}\right)$ is sufficient to identify $g$ and that:

(i) $\mathbb{P}\left(T^{*}=1 \mid \mathbf{x}, z=0\right) \neq \mathbb{P}\left(T^{*}=1 \mid \mathbf{x}, z=1\right)$. 
(ii) $T$ is conditionally independent of z given $\left(\mathbf{x}, T^{*}\right)$.

(iii) $\alpha_{0}(\mathbf{x})+\alpha_{1}(\mathbf{x})<1$

(iv) $\mathbb{E}\left[v \mid \mathbf{x}, z, T^{*}, T\right]=0$

(v) $g(1, \mathbf{x}) \neq g(0, \mathbf{x})$

Theorem B.1 (Mahajan (2006) Theorem 1). Under Assumption B.1, $\alpha_{0}(\mathbf{x})$ and $\alpha_{1}(\mathbf{x})$ are point identified, as is $g\left(T^{*}, \mathbf{x}\right)$.

Assumption B.1 (i) is equivalent to our Assumption 2.1 (ii), while Assumptions B.1 (ii)-(iii) are equivalent to our Assumptions 2.2 (i)-(ii). Assumption B.1 (v) serves the same purpose as $\beta(\mathbf{x}) \neq 0$ in our Theorem 2.3: unless $T^{*}$ affects $y$, we cannot identify the misclassification probabilities. The key difference between Theorem B.1 and the setting we consider in Section 2 comes from Assumption B.1 (iv). This is essentially a stronger version of our Assumptions 2.1 (iii) and 2.2 (iii) but applies to the projection error $v$, defined in Assumption B.1 rather than the structural error $\varepsilon$, defined in Assumption 2.1 (i). Accordingly, Theorem B.1 identifies the conditional mean function $g$ rather than the causal effect $\beta(\mathbf{x})$.

Although the meaning of the error term changes when we move from a structural to a reduced form model, the meaning of the mis-classification error rates does not: $\alpha_{0}(\mathbf{x})$ and $\alpha_{1}(\mathbf{x})$ are simply conditional probabilities for $T$ given $\left(T^{*}, \mathbf{x}\right)$. Step 2 of Mahajan (2006) A.2 relies on this insight. The idea is to find a way to satisfy Assumption B.1 (iv) simultaneously with Assumptions 2.1 (iii) and 2.2 (iii), while allowing $T^{*}$ to be endogenous. If this can be achieved, $\alpha_{0}(\mathbf{x}), \alpha_{1}(\mathbf{x})$ will be identified via Theorem B.1, and identification of $\beta(\mathbf{x})$ will follow from step 1 of A.2 (our Lemma 2.2). To this end, Mahajan (2006) invokes the condition

$$
\mathbb{E}\left(y \mid \mathbf{x}, z, T^{*}, T\right)=\mathbb{E}\left(y \mid \mathbf{x}, T^{*}\right) .
$$

Because Mahajan (2006) A.2 assumes an additively separable model - our Assumption 2.1 (i) - we see that

$$
\mathbb{E}\left(y \mid \mathbf{x}, z, T^{*}, T\right)=c(\mathbf{x})+\beta(\mathbf{x}) T^{*}+\mathbb{E}\left(\varepsilon \mid \mathbf{x}, z, T^{*}, T\right)
$$

so Equation B.1 is equivalent to $\mathbb{E}\left(\varepsilon \mid \mathbf{x}, z, T^{*}, T\right)=\mathbb{E}\left(\varepsilon \mid \mathbf{x}, T^{*}\right)$. Note that this allows $T^{*}$ to be endogenous, as it does not require $\mathbb{E}\left(\varepsilon \mid \mathbf{x}, T^{*}\right)=0$. Now, applying Equation B.1 to the definition of $v$ from Assumption B.1, we have

$$
\mathbb{E}\left(v \mid \mathbf{x}, z, T^{*}, T\right)=\mathbb{E}\left[y-\mathbb{E}\left(y \mid \mathbf{x}, T^{*}\right) \mid \mathbf{x}, z, T^{*}, T\right]=0
$$

which satisfies Assumption B.1 (iv) as required. Based on this reasoning, Mahajan (2006) claims that Equation B.1 along with Assumptions B.1 (iv), 2.1, and 2.2 (i)-(ii) suffice to identify the effect $\beta(\mathbf{x})$ of an endogenous $T^{*}$, so long as $g(1, \mathbf{x}) \neq g(0, \mathbf{x})$. As we now show, however, these Assumptions are contradictory unless $T^{*}$ is exogenous.

By Equation B.1 and Assumption 2.1 (i), $\mathbb{E}\left(\varepsilon \mid \mathbf{x}, z, T^{*}, T\right)=\mathbb{E}\left(\varepsilon \mid \mathbf{x}, T^{*}\right)$ and thus by iterated expectations, we obtain

$$
\mathbb{E}\left(\varepsilon \mid \mathbf{x}, T^{*}, z\right)=\mathbb{E}_{T \mid \mathbf{x}, T^{*}, z}\left[\mathbb{E}\left(\varepsilon \mid \mathbf{x}, T^{*}, T, z\right)\right]=\mathbb{E}_{T \mid \mathbf{x}, T^{*}, z}\left[\mathbb{E}\left(\varepsilon \mid \mathbf{x}, T^{*}\right)\right]=\mathbb{E}\left(\varepsilon \mid \mathbf{x}, T^{*}\right) .
$$


Now, let $m_{t k}^{*}(\mathbf{x})=\mathbb{E}\left(\varepsilon \mid \mathbf{x}, T^{*}=t, z=k\right)$. Using this notation, Equation B.2 is equivalent to $m_{t 0}^{*}(\mathbf{x})=m_{t 1}^{*}(\mathbf{x})$ for $t=0,1$. Combining iterated expectations with Assumption 2.1 (iii),

$$
\mathbb{E}(\varepsilon \mid \mathbf{x}, z=k)=\left[1-p_{k}^{*}(\mathbf{x})\right] m_{0 k}^{*}(\mathbf{x})+p_{k}^{*}(\mathbf{x}) m_{1 k}^{*}(\mathbf{x})=0
$$

for $k=0,1$ where $p_{k}^{*}(\mathbf{x}) \equiv \mathbb{P}\left(T^{*}=1 \mid \mathbf{x}, z=k\right)$. But substituting $m_{t 0}^{*}(\mathbf{x})=m_{t 1}^{*}(\mathbf{x})$ into Equation B.3 for $k=0,1$, we obtain

$$
\begin{aligned}
& {\left[1-p_{0}^{*}(\mathbf{x})\right] m_{00}^{*}(\mathbf{x})+p_{0}^{*}(\mathbf{x}) m_{10}^{*}(\mathbf{x})=0} \\
& {\left[1-p_{1}^{*}(\mathbf{x})\right] m_{00}^{*}(\mathbf{x})+p_{1}^{*}(\mathbf{x}) m_{10}^{*}(\mathbf{x})=0}
\end{aligned}
$$

The preceding two equalities are convex combinations of $m_{00}^{*}$ and $m_{10}^{*}$. The only way that both can equal zero simultaneously is if either $p_{0}^{*}(\mathbf{x})=p_{1}^{*}(\mathbf{x})$, contradicting Assumption 2.1 (ii), or if $m_{t k}^{*}(\mathbf{x})=0$ for all $(t, k)$, which implies that $T^{*}$ is exogenous. Hence Mahajan (2006) A.2 fails: given the assumption that $z$ is a valid instrument for $\varepsilon$, Equation B.1 implies that either there is no first-stage relationship between $z$ and $T^{*}$ or that $T^{*}$ is exogenous.

The root of the problem with A.2 is the attempt to use one instrument to satisfy both the assumptions of Theorem B.1 and Lemma 2.2. If one had access to a second instrument $w$, or equivalently a second mis-measured surrogate for $T^{*}$, that satisfied Assumptions B.1, one could use $w$ to recover $\alpha_{0}(\mathbf{x})$ and $\alpha_{1}(\mathbf{x})$ via Theorem B.1 and $z$ to recover the IV estimand $\beta(\mathbf{x}) /\left[1-\alpha_{0}(\mathbf{x})-\alpha_{1}(\mathbf{x})\right]$ via Lemma 2.2. This is effectively the approach used by Battistin et al. (2014) to evaluate the returns to schooling in a setting with multiple misreported measures of educational qualifications.

\section{Moment Equalities Under Joint Exogeneity}

In this Section we discuss the moment equalities that replace Equation 10 under joint exogeneity: Assumption 2.3. Because the moment inequalities from Section 3.3 are unchanged under this assumption, we do not discuss them further here. Define $\theta_{1}$ as in Equation $6, \kappa_{1}$ as in Section 3.1, and let $\rho=-\theta_{1} \alpha_{0}\left(1-\alpha_{1}\right)$ and $\eta=\theta_{1}\left(1+\alpha_{0}-\alpha_{1}\right)$. Now, under Assumptions $2.1,2.2$, and 2.3:

$$
\mathbb{E}\left\{\left[\begin{array}{c}
y-\kappa_{1}-\theta_{1} T \\
\left(y-\kappa_{1}\right) T-\rho-\eta T
\end{array}\right] \otimes\left[\begin{array}{l}
1 \\
z
\end{array}\right]\right\}=\mathbf{0} .
$$

where the equalities involving $\rho$ and $\eta$ follow from an argument similar to one of the steps from the proof of Lemma 2.3 - see, e.g., Frazis and Loewenstein (2003) and Mahajan (2006). The moment equalities from C.1 point identify the reduced form parameters $\left(\theta_{1}, \kappa_{1}, \rho, \eta\right)$ and lead to a just-identified method of moments estimator of the same. To see why knowledge of $\left(\theta_{1}, \kappa_{1}, \rho, \eta\right)$ suffices to identify $\left(\beta, \alpha_{0}, \alpha_{1}\right)$, define

$$
A \equiv \eta / \theta_{1}=1+\alpha_{0}-\alpha_{1}, \quad B \equiv-\rho / \theta_{1}=\alpha_{0}\left(1-\alpha_{1}\right)
$$

Eliminating $\left(1-\alpha_{1}\right)$ and $\alpha_{0}$, respectively, we obtain:

$$
\alpha_{0}^{2}-A \alpha_{0}+B=0, \quad\left(1-\alpha_{1}\right)^{2}-A\left(1-\alpha_{1}\right)+B=0
$$


These are exactly the same quadratic, namely $x^{2}-A x+B=0$. Hence one root is $\alpha_{0}$ while the other is $\left(1-\alpha_{1}\right)$. The discriminant is

$$
A^{2}-4 B=\left[\left(1-\alpha_{1}\right)+\alpha_{0}\right]^{2}-4\left[\alpha_{0}\left(1-\alpha_{1}\right)\right]=\left(1-\alpha_{0}-\alpha_{1}\right)^{2}
$$

so that both roots are real as long as $\alpha_{0}+\alpha_{1} \neq 0$. To solve for $\alpha_{0}$ and $\alpha_{1}$ we need to calculate the roots of $x^{2}-A x+B=0$, namely $x=\frac{1}{2}\left(A \pm \sqrt{A^{2}-4 B}\right)$. One of these roots is $\alpha_{0}$ and the other is $1-\alpha_{1}$. By assumption, however, $\alpha_{0}+\alpha_{1}<1$ and thus $\alpha_{0}<1-\alpha_{1}$. It follows that the smaller of the two roots is $\alpha_{0}$ and the larger is $1-\alpha_{1}$. Given that $\left(\alpha_{0}, \alpha_{1}\right)$ are identified, identification of $\beta$ follows by Lemma 2.2.

Inference based on the moment equalities from Equation C.1 suffers from the same difficulties as that based on Equation 10 above. First, note that, while $A^{2}>4 B$ in population since $\alpha_{0}+\alpha_{1}<1$ by assumption, the same may not hold in sample. In this case the GMM estimator of $\beta$ will fail to exist. Second, notice that the moment equalities from Equation C.1 only depend on $\beta$ through $\theta_{1}$ and are completely uninformative about $\left(\alpha_{0}, \alpha_{1}\right)$ if $\beta=0$.

Substituting Equation C.1 for Equation 10 in Algorithm 3.1 requires some small changes. First, $m^{E}$ and $h^{E}$ from Equations 16-17 are replaced by

$$
h^{E}=\left[\begin{array}{c}
y-\kappa_{1}-\theta_{1} T \\
\left(y-\kappa_{1}-\theta_{1} T\right) z
\end{array}\right], \quad m^{E}=\left[\begin{array}{c}
\left(y-\kappa_{1}\right) T-\rho-\eta T \\
\left\{\left(y-\kappa_{1}\right) T-\rho-\eta T\right\} z
\end{array}\right]
$$

where in this case we require preliminary estimators of $\kappa_{1}$ and $\theta_{1}$. Accordingly, $H^{E}$ and $M^{E}$ from Lemma 3.1 become

$$
H^{E}=\left[\begin{array}{cc}
-1 & -\mathbb{E}(T) \\
-\mathbb{E}(z) & -\mathbb{E}(T z)
\end{array}\right], \quad M^{E}=\left[\begin{array}{cc}
-\mathbb{E}[T] & 0 \\
-\mathbb{E}[T z] & 0
\end{array}\right]
$$

and thus

$$
B^{E}=-M^{E}\left(H^{E}\right)^{-1}=\frac{1}{\operatorname{Cov}(T, z)}\left[\begin{array}{cc}
-\mathbb{E}(T) \mathbb{E}(T z) & \mathbb{E}(T)^{2} \\
-\mathbb{E}(T z)^{2} & \mathbb{E}(T z) \mathbb{E}(T)
\end{array}\right]
$$

which is well-defined as long as $T$ is correlated with $z$.

\section{References}

Aigner, D. J., 1973. Regression with a binary independent variable subject to errors of observation. Journal of Econometrics 1, 49-60.

Andrews, D. W., 1994. Empirical process methods in econometrics. Handbook of econometrics 4, $2247-2294$.

Andrews, D. W., Shi, X., 2013. Inference based on conditional moment inequalities. Econometrica $81(2), 609-666$.

Andrews, D. W., Shi, X., 2014. Nonparametric inference based on conditional moment inequalities. Journal of Econometrics 179 (1), 31-45.

Andrews, D. W., Soares, G., 2010. Inference for parameters defined by moment inequalities using generalized moment selection. Econometrica 78 (1), 119-157. 
Andrews, I., 2016. Valid two-step identification-robust confidence sets for GMM. Review of Economics and Statistics (Forthcoming).

Battistin, E., Nadai, M. D., Sianesi, B., 2014. Misreported schooling, multiple measures and returns to educational qualifications. Journal of Econometrics 181 (2), 136-150.

Black, D. A., Berger, M. C., Scott, F. A., 2000. Bounding parameter estimates with nonclassical measurement error. Journal of the American Statistical Association 95 (451), 739-748.

Bollinger, C. R., 1996. Bounding mean regressions when a binary regressor is mismeasured. Journal of Econometrics 73, 387-399.

Bollinger, C. R., van Hasselt, M., 2015. Bayesian moment-based inference in a regression models with misclassification error, working Paper.

Bugni, F. A., Canay, I. A., Shi, X., 2017. Inference for subvectors and other functions of partially identified parameters in moment inequality models. Quantitative Economics 8 (1), 1-38.

Chen, X., Hong, H., Nekipelov, D., 2011. Nonlinear models of measurement errors. Journal of Economic Literature 49 (4), 901-937.

Chen, X., Hong, H., Tamer, E., 2005. Measurement error models with auxiliary data. The Review of Economic Studies 72 (2), 343-366.

Chen, X., Hu, Y., Lewbel, A., 2008a. Nonparametric identification of regression models containing a misclassified dichotomous regressor with instruments. Economics Letters 100, 381-384.

Chen, X., Hu, Y., Lewbel, A., 2008b. A note on the closed-form identification of regression models with a mismeasured binary regressor. Statistics \& Probability Letters 78 (12), 1473-1479.

Frazis, H., Loewenstein, M. A., 2003. Estimating linear regressions with mismeasured, possibly endogenous, binary explanatory variables. Journal of Econometrics 117, 151-178.

$\mathrm{Hu}, \mathrm{Y} ., 2$ 2008. Identification and estimation of nonlinear models with misclassification error using instrumental variables: A general solution. Journal of Econometrics 144 (1), 27-61.

Hu, Y., Shennach, S. M., January 2008. Instrumental variable treatment of nonclassical measurement error models. Econometrica 76 (1), 195-216.

Hu, Y., Shiu, J.-L., Woutersen, T., 2015. Identification and estimation of single-index models with measurement error and endogeneity. The Econometrics Journal 18 (3), 347-362.

Kaido, H., Molinari, F., Stoye, J., 2016. Confidence intervals for projections of partially identified parameters. arXiv preprint arXiv:1601.00934.

Kane, T. J., Rouse, C. E., Staiger, D., July 1999. Estimating returns to schooling when schooling is misreported. Tech. rep., National Bureau of Economic Research, NBER Working Paper 7235.

Kreider, B., Pepper, J. V., Gundersen, C., Jolliffe, D., 2012. Identifying the effects of SNAP (food stamps) on child health outcomes when participation is endogenous and misreported. Journal of the American Statistical Association 107 (499), 958-975. 
Lewbel, A., March 2007. Estimation of average treatment effects with misclassification. Econometrica $75(2), 537-551$.

Mahajan, A., 2006. Identification and estimation of regression models with misclassification. Econometrica $74(3), 631-665$.

Molinari, F., 2008. Partial identification of probability distributions with misclassified data. Journal of Econometrics 144 (1), 81-117.

Moon, H. R., Schorfheide, F., 2009. Estimation with overidentifying inequality moment conditions. Journal of Econometrics 153 (2), 136-154.

Newey, W. K., McFadden, D., 1994. Large sample estimation and hypothesis testing. Handbook of econometrics 4, 2111-2245.

Nguimkeu, P., Denteh, A., Tchernis, R., 2016. On the estimation of treatment effects with endogenous misreporting. Working Paper.

Schennach, S. M., 2004. Estimation of nonlinear models with measurement error. Econometrica $72(1), 33-75$.

Schennach, S. M., 2007. Instrumental variable estimation of nonlinear errors-in-variables models. Econometrica 75 (1), 201-239.

Schennach, S. M., 2013. Measurement error in nonlinear models - a review. In: Acemoglu, D., Arellano, M., Dekel, E. (Eds.), Advances in Economics and Econometrics. Vol. 3. Cambridge University Press, pp. 296-337.

Shiu, J.-L., 2015. Identification and estimation of endogenous selection models in the presence of misclassification errors. Economic Modelling (Forthcoming).

Song, S., 2015. Semiparametric estimation of models with conditional moment restrictions in the presence of nonclassical measurement errors. Journal of Econometrics 185 (1), 95-109.

Song, S., Schennach, S. M., White, H., 2015. Estimating nonseparable models with mismeasured endogenous variables. Quantitative Economics 6 (3), 749-794.

Ura, T., November 2015. Heterogeneous treatment effects with mismeasured endogenous treatment. Tech. rep., Duke University Department of Economics.

van Hasselt, M., Bollinger, C. R., 2012. Binary misclassification and identification in regression models. Economics Letters 115, 81-84. 


\section{Supplementary Simulation Results: Online Only}

In this section we provide additional simulation results to supplement those from Section 4 above. For details of the simulation DGP, etc. see the discussion above.

\begin{tabular}{rr|rrrrrrrr}
\hline \hline & & \multicolumn{1}{|c}{$\beta$} \\
$\alpha_{0}$ & $\alpha_{1}$ & 0 & 0.25 & 0.5 & 0.75 & 1 & 1.5 & 2 & 3 \\
\hline 0.0 & 0.0 & 90 & 90 & 90 & 91 & 90 & 91 & 90 & 90 \\
& 0.1 & 91 & 93 & 94 & 94 & 94 & 94 & 90 & 89 \\
& 0.2 & 92 & 93 & 94 & 94 & 94 & 94 & 92 & 90 \\
& 0.3 & 93 & 93 & 94 & 94 & 94 & 93 & 92 & 91 \\
\hline 0.1 & 0.0 & 92 & 93 & 93 & 94 & 94 & 93 & 90 & 87 \\
& 0.1 & 93 & 95 & 96 & 97 & 97 & 96 & 92 & 87 \\
& 0.2 & 95 & 96 & 97 & 98 & 97 & 96 & 92 & 87 \\
& 0.3 & 96 & 98 & 98 & 98 & 98 & 95 & 92 & 88 \\
\hline 0.2 & 0.0 & 93 & 93 & 93 & 93 & 93 & 93 & 92 & 89 \\
& 0.1 & 95 & 96 & 98 & 98 & 97 & 95 & 93 & 89 \\
& 0.2 & 97 & 97 & 98 & 98 & 97 & 95 & 92 & 89 \\
& 0.3 & 98 & 98 & 98 & 98 & 97 & 95 & 93 & 91 \\
\hline 0.3 & 0.0 & 93 & 94 & 94 & 94 & 94 & 93 & 92 & 91 \\
& 0.1 & 97 & 97 & 98 & 98 & 97 & 95 & 93 & 89 \\
& 0.2 & 98 & 98 & 98 & 98 & 97 & 94 & 93 & 91 \\
& 0.3 & 99 & 99 & 99 & 98 & 98 & 96 & 95 & 94 \\
\hline
\end{tabular}

Table D.1: Coverage $(1-$ size $)$ of $90 \%$ GMS joint test for $\alpha_{0}$ and $\alpha_{1}: n=1000$. 


\begin{tabular}{rr|rrrrrrrr}
\hline \hline & & \multicolumn{1}{|c}{$\beta$} \\
$\alpha_{0}$ & $\alpha_{1}$ & 0 & 0.25 & 0.5 & 0.75 & 1 & 1.5 & 2 & 3 \\
\hline 0.0 & 0.0 & 90 & 91 & 91 & 90 & 90 & 90 & 90 & 90 \\
& 0.1 & 91 & 92 & 92 & 93 & 94 & 94 & 92 & 90 \\
& 0.2 & 91 & 92 & 93 & 93 & 93 & 94 & 93 & 91 \\
& 0.3 & 92 & 93 & 93 & 93 & 94 & 93 & 93 & 91 \\
\hline 0.1 & 0.0 & 90 & 92 & 93 & 94 & 93 & 94 & 92 & 89 \\
& 0.1 & 92 & 93 & 95 & 96 & 97 & 97 & 94 & 90 \\
& 0.2 & 92 & 94 & 96 & 97 & 97 & 96 & 95 & 89 \\
& 0.3 & 94 & 95 & 97 & 98 & 98 & 96 & 94 & 90 \\
\hline 0.2 & 0.0 & 91 & 93 & 93 & 93 & 93 & 94 & 92 & 90 \\
& 0.1 & 92 & 95 & 96 & 97 & 97 & 96 & 94 & 90 \\
& 0.2 & 94 & 96 & 97 & 97 & 97 & 95 & 93 & 90 \\
& 0.3 & 96 & 97 & 98 & 98 & 97 & 95 & 93 & 90 \\
\hline 0.3 & 0.0 & 92 & 92 & 93 & 93 & 93 & 93 & 92 & 91 \\
& 0.1 & 93 & 96 & 97 & 97 & 97 & 96 & 93 & 90 \\
& 0.2 & 96 & 97 & 97 & 97 & 96 & 95 & 93 & 90 \\
& 0.3 & 98 & 98 & 98 & 98 & 97 & 95 & 94 & 92 \\
\hline
\end{tabular}

Table D.2: Coverage $(1-$ size $)$ of $90 \%$ GMS joint test for $\alpha_{0}$ and $\alpha_{1}: n=2000$

\begin{tabular}{rr|rrrrrrrr}
\hline \hline & & \multicolumn{1}{|c}{$\beta$} \\
$\alpha_{0}$ & $\alpha_{1}$ & 0 & 0.25 & 0.5 & 0.75 & 1 & 1.5 & 2 & 3 \\
\hline 0.0 & 0.0 & 95 & 95 & 95 & 96 & 96 & 96 & 95 & 95 \\
& 0.1 & 96 & 97 & 97 & 97 & 97 & 97 & 95 & 94 \\
& 0.2 & 96 & 97 & 98 & 98 & 97 & 97 & 96 & 95 \\
& 0.3 & 97 & 97 & 97 & 98 & 97 & 97 & 96 & 95 \\
\hline 0.1 & 0.0 & 96 & 97 & 97 & 97 & 97 & 97 & 95 & 93 \\
& 0.1 & 97 & 98 & 99 & 99 & 99 & 98 & 96 & 92 \\
& 0.2 & 98 & 99 & 99 & 99 & 99 & 98 & 96 & 93 \\
& 0.3 & 99 & 99 & 99 & 99 & 99 & 98 & 96 & 94 \\
\hline 0.2 & 0.0 & 97 & 97 & 97 & 97 & 97 & 96 & 96 & 94 \\
& 0.1 & 98 & 99 & 99 & 99 & 99 & 98 & 96 & 94 \\
& 0.2 & 99 & 99 & 99 & 99 & 99 & 98 & 96 & 94 \\
& 0.3 & 99 & 100 & 100 & 99 & 99 & 98 & 97 & 95 \\
\hline 0.3 & 0.0 & 97 & 97 & 97 & 97 & 97 & 96 & 96 & 95 \\
& 0.1 & 99 & 99 & 99 & 99 & 99 & 98 & 97 & 94 \\
& 0.2 & 99 & 99 & 99 & 99 & 99 & 98 & 97 & 96 \\
& 0.3 & 100 & 100 & 100 & 99 & 99 & 98 & 98 & 97 \\
\hline
\end{tabular}

Table D.3: Coverage $(1-$ size $)$ of $95 \%$ GMS joint test for $\alpha_{0}$ and $\alpha_{1}: n=1000$ 


\begin{tabular}{rr|rrrrrrrr}
\hline \hline & & \multicolumn{1}{|c}{$\beta$} \\
$\alpha_{0}$ & $\alpha_{1}$ & 0 & 0.25 & 0.5 & 0.75 & 1 & 1.5 & 2 & 3 \\
\hline 0.0 & 0.0 & 95 & 95 & 96 & 95 & 95 & 95 & 95 & 95 \\
& 0.1 & 96 & 96 & 96 & 97 & 97 & 97 & 96 & 95 \\
& 0.2 & 96 & 96 & 97 & 97 & 97 & 97 & 96 & 95 \\
& 0.3 & 96 & 97 & 97 & 97 & 97 & 97 & 97 & 95 \\
\hline 0.1 & 0.0 & 95 & 96 & 97 & 97 & 97 & 97 & 96 & 94 \\
& 0.1 & 96 & 97 & 98 & 98 & 99 & 99 & 97 & 94 \\
& 0.2 & 96 & 98 & 98 & 99 & 99 & 98 & 97 & 94 \\
& 0.3 & 97 & 98 & 99 & 99 & 99 & 98 & 97 & 95 \\
\hline 0.2 & 0.0 & 96 & 96 & 97 & 97 & 97 & 97 & 96 & 95 \\
& 0.1 & 96 & 98 & 98 & 99 & 99 & 98 & 97 & 94 \\
& 0.2 & 97 & 98 & 99 & 99 & 99 & 98 & 97 & 95 \\
& 0.3 & 98 & 99 & 99 & 99 & 99 & 98 & 97 & 94 \\
\hline 0.3 & 0.0 & 96 & 96 & 97 & 97 & 97 & 97 & 96 & 95 \\
& 0.1 & 97 & 98 & 99 & 99 & 99 & 98 & 96 & 94 \\
& 0.2 & 98 & 99 & 99 & 99 & 98 & 98 & 96 & 95 \\
& 0.3 & 99 & 99 & 99 & 99 & 99 & 98 & 97 & 96 \\
\hline
\end{tabular}

Table D.4: Coverage $(1-$ size $)$ of $95 \%$ GMS joint test for $\alpha_{0}$ and $\alpha_{1}: n=2000$

\begin{tabular}{rr|rrrrrrrr}
\hline \hline & & \multicolumn{1}{|c}{$\beta$} \\
$\alpha_{0}$ & $\alpha_{1}$ & 0 & 0.25 & 0.5 & 0.75 & 1 & 1.5 & 2 & 3 \\
\hline 0.0 & 0.0 & 97.7 & 97.7 & 97.6 & 97.7 & 98.0 & 98.0 & 97.4 & 97.9 \\
& 0.1 & 98.0 & 98.7 & 98.8 & 99.1 & 98.8 & 98.4 & 97.1 & 96.4 \\
& 0.2 & 98.4 & 98.5 & 98.9 & 98.9 & 98.8 & 98.6 & 98.0 & 97.0 \\
& 0.3 & 98.5 & 98.8 & 98.8 & 99.0 & 98.7 & 98.4 & 97.8 & 97.5 \\
\hline 0.1 & 0.0 & 98.1 & 98.5 & 98.3 & 98.8 & 98.8 & 98.4 & 96.8 & 95.7 \\
& 0.1 & 98.6 & 99.1 & 99.5 & 99.6 & 99.6 & 98.8 & 97.7 & 95.2 \\
& 0.2 & 99.0 & 99.3 & 99.7 & 99.8 & 99.7 & 98.9 & 97.5 & 95.7 \\
& 0.3 & 99.4 & 99.7 & 99.8 & 99.8 & 99.6 & 99.0 & 98.2 & 96.7 \\
\hline 0.2 & 0.0 & 98.6 & 98.5 & 98.6 & 98.9 & 98.7 & 98.2 & 97.7 & 97.0 \\
& 0.1 & 99.0 & 99.5 & 99.7 & 99.7 & 99.4 & 99.0 & 98.1 & 96.5 \\
& 0.2 & 99.5 & 99.7 & 99.8 & 99.7 & 99.4 & 99.0 & 97.8 & 96.8 \\
& 0.3 & 99.7 & 99.8 & 99.8 & 99.8 & 99.5 & 99.0 & 98.7 & 97.7 \\
\hline 0.3 & 0.0 & 98.7 & 98.7 & 98.8 & 98.7 & 98.7 & 98.2 & 98.1 & 97.6 \\
& 0.1 & 99.4 & 99.6 & 99.6 & 99.7 & 99.4 & 98.9 & 98.3 & 96.8 \\
& 0.2 & 99.8 & 99.8 & 99.7 & 99.8 & 99.5 & 99.1 & 98.5 & 97.8 \\
& 0.3 & 100.0 & 99.9 & 99.9 & 99.8 & 99.6 & 99.5 & 99.1 & 98.8 \\
\hline
\end{tabular}

Table D.5: Coverage $(1-$ size $)$ of $97.5 \%$ GMS joint test for $\alpha_{0}$ and $\alpha_{1}: n=1000$ 


\begin{tabular}{rr|rrrrrrrr}
\hline \hline & & \multicolumn{1}{|c}{$\beta$} \\
$\alpha_{0}$ & $\alpha_{1}$ & 0 & 0.25 & 0.5 & 0.75 & 1 & 1.5 & 2 & 3 \\
\hline 0.0 & 0.0 & 97.7 & 97.7 & 97.6 & 97.6 & 97.6 & 97.5 & 97.4 & 97.5 \\
& 0.1 & 98.0 & 98.1 & 98.4 & 98.3 & 98.8 & 98.6 & 97.8 & 97.0 \\
& 0.2 & 98.1 & 98.2 & 98.8 & 98.6 & 98.9 & 98.6 & 98.3 & 97.3 \\
& 0.3 & 98.2 & 98.5 & 98.6 & 98.6 & 98.8 & 98.4 & 98.2 & 97.4 \\
\hline 0.1 & 0.0 & 97.4 & 98.1 & 98.3 & 98.8 & 98.5 & 98.5 & 97.9 & 96.9 \\
& 0.1 & 98.0 & 98.6 & 99.1 & 99.4 & 99.5 & 99.3 & 98.4 & 96.8 \\
& 0.2 & 98.2 & 98.9 & 99.4 & 99.6 & 99.7 & 99.3 & 98.8 & 96.8 \\
& 0.3 & 98.6 & 99.1 & 99.6 & 99.8 & 99.6 & 99.2 & 98.4 & 97.0 \\
\hline 0.2 & 0.0 & 97.8 & 98.1 & 98.5 & 98.6 & 98.5 & 98.4 & 98.0 & 97.6 \\
& 0.1 & 98.3 & 98.9 & 99.2 & 99.6 & 99.5 & 99.1 & 98.6 & 97.0 \\
& 0.2 & 98.7 & 99.4 & 99.7 & 99.6 & 99.5 & 99.0 & 98.4 & 96.9 \\
& 0.3 & 99.1 & 99.6 & 99.7 & 99.7 & 99.5 & 99.0 & 98.2 & 97.0 \\
\hline 0.3 & 0.0 & 98.2 & 98.3 & 98.7 & 98.5 & 98.6 & 98.5 & 98.0 & 97.7 \\
& 0.1 & 98.6 & 99.3 & 99.4 & 99.6 & 99.5 & 99.2 & 98.1 & 97.0 \\
& 0.2 & 99.2 & 99.7 & 99.7 & 99.6 & 99.4 & 98.8 & 98.4 & 97.4 \\
& 0.3 & 99.6 & 99.8 & 99.8 & 99.7 & 99.4 & 99.1 & 98.8 & 98.2 \\
\hline
\end{tabular}

Table D.6: Coverage $(1-$ size $)$ of $97.5 \%$ GMS joint test for $\alpha_{0}$ and $\alpha_{1}: n=2000$

\begin{tabular}{rr|rrrrrrrr}
\hline \hline & & & & \multicolumn{1}{|c}{$\beta$} \\
$\alpha_{0}$ & $\alpha_{1}$ & 0 & 0.25 & 0.5 & 0.75 & 1 & 1.5 & 2 & 3 \\
\hline 0.0 & 0.0 & 27 & 33 & 30 & 14 & 1 & 0 & 0 & 0 \\
& 0.1 & 27 & 32 & 29 & 13 & 2 & 0 & 0 & 0 \\
& 0.2 & 26 & 33 & 32 & 15 & 4 & 0 & 0 & 0 \\
& 0.3 & 26 & 34 & 30 & 17 & 5 & 0 & 0 & 0 \\
\hline 0.1 & 0.0 & 26 & 32 & 31 & 14 & 2 & 0 & 0 & 0 \\
& 0.1 & 26 & 36 & 32 & 16 & 4 & 0 & 0 & 0 \\
& 0.2 & 27 & 35 & 31 & 18 & 8 & 0 & 0 & 0 \\
& 0.3 & 25 & 35 & 32 & 21 & 11 & 1 & 0 & 0 \\
\hline 0.2 & 0.0 & 26 & 33 & 30 & 15 & 3 & 0 & 0 & 0 \\
& 0.1 & 26 & 33 & 30 & 19 & 6 & 0 & 0 & 0 \\
& 0.2 & 26 & 35 & 33 & 22 & 12 & 1 & 0 & 0 \\
& 0.3 & 26 & 35 & 33 & 26 & 15 & 3 & 0 & 0 \\
\hline 0.3 & 0.0 & 26 & 32 & 32 & 16 & 6 & 0 & 0 & 0 \\
& 0.1 & 24 & 35 & 33 & 21 & 11 & 1 & 0 & 0 \\
& 0.2 & 26 & 32 & 35 & 27 & 15 & 4 & 0 & 0 \\
& 0.3 & 26 & 35 & 35 & 28 & 21 & 7 & 2 & 0 \\
\hline
\end{tabular}

Table D.7: Percentage of simulation replications for which the standard GMM confidence interval fails to exist, either becuase the point estimate is $\mathrm{NaN}$ or the asymptotic covariance matrix is numerically singular $(n=1000)$ 


\begin{tabular}{rr|rrrrrrrr}
\hline \hline & & & & \multicolumn{1}{|c}{$\beta$} & & & & \\
$\alpha_{0}$ & $\alpha_{1}$ & 0 & 0.25 & 0.5 & 0.75 & 1 & 1.5 & 2 & 3 \\
\hline 0.0 & 0.0 & 25 & 36 & 29 & 7 & 0 & 0 & 0 & 0 \\
& 0.1 & 28 & 36 & 29 & 7 & 0 & 0 & 0 & 0 \\
& 0.2 & 28 & 37 & 28 & 10 & 1 & 0 & 0 & 0 \\
& 0.3 & 27 & 36 & 28 & 12 & 2 & 0 & 0 & 0 \\
\hline 0.1 & 0.0 & 27 & 36 & 27 & 10 & 0 & 0 & 0 & 0 \\
& 0.1 & 26 & 36 & 29 & 9 & 1 & 0 & 0 & 0 \\
& 0.2 & 28 & 38 & 29 & 13 & 2 & 0 & 0 & 0 \\
& 0.3 & 24 & 36 & 31 & 15 & 5 & 0 & 0 & 0 \\
\hline 0.2 & 0.0 & 26 & 36 & 30 & 9 & 1 & 0 & 0 & 0 \\
& 0.1 & 25 & 37 & 29 & 12 & 2 & 0 & 0 & 0 \\
& 0.2 & 27 & 38 & 32 & 17 & 4 & 0 & 0 & 0 \\
& 0.3 & 25 & 39 & 34 & 20 & 9 & 1 & 0 & 0 \\
\hline 0.3 & 0.0 & 26 & 37 & 30 & 10 & 2 & 0 & 0 & 0 \\
& 0.1 & 25 & 38 & 31 & 16 & 4 & 0 & 0 & 0 \\
& 0.2 & 27 & 38 & 34 & 19 & 9 & 0 & 0 & 0 \\
& 0.3 & 27 & 36 & 36 & 23 & 13 & 2 & 0 & 0 \\
\hline
\end{tabular}

Table D.8: Percentage of simulation replications for which the standard GMM confidence interval fails to exist, either becuase the point estimate is $\mathrm{NaN}$ or the asymptotic covariance matrix is numerically singular $(n=2000)$

\begin{tabular}{rr|rrrrrrrr}
\hline \hline & & & & \multicolumn{1}{|c}{$\beta$} & & & & \\
$\alpha_{0}$ & $\alpha_{1}$ & 0 & 0.25 & 0.5 & 0.75 & 1 & 1.5 & 2 & 3 \\
\hline 0.0 & 0.0 & 72 & 62 & 62 & 80 & 92 & 95 & 94 & 95 \\
& 0.1 & 72 & 62 & 63 & 79 & 92 & 95 & 96 & 95 \\
& 0.2 & 73 & 61 & 61 & 77 & 90 & 96 & 96 & 96 \\
& 0.3 & 73 & 59 & 62 & 76 & 88 & 95 & 96 & 95 \\
\hline 0.1 & 0.0 & 73 & 63 & 60 & 78 & 91 & 95 & 96 & 96 \\
& 0.1 & 73 & 58 & 59 & 77 & 90 & 95 & 95 & 94 \\
& 0.2 & 73 & 59 & 61 & 75 & 86 & 95 & 95 & 94 \\
& 0.3 & 74 & 59 & 58 & 71 & 82 & 94 & 96 & 96 \\
\hline 0.2 & 0.0 & 74 & 62 & 60 & 78 & 91 & 95 & 96 & 96 \\
& 0.1 & 73 & 60 & 61 & 74 & 87 & 95 & 96 & 94 \\
& 0.2 & 73 & 58 & 57 & 70 & 81 & 93 & 95 & 95 \\
& 0.3 & 73 & 58 & 56 & 66 & 78 & 92 & 95 & 96 \\
\hline 0.3 & 0.0 & 74 & 62 & 60 & 76 & 89 & 95 & 96 & 96 \\
& 0.1 & 75 & 59 & 58 & 71 & 82 & 93 & 96 & 95 \\
& 0.2 & 74 & 61 & 56 & 65 & 78 & 90 & 96 & 96 \\
& 0.3 & 73 & 58 & 55 & 64 & 71 & 88 & 93 & 96 \\
\hline
\end{tabular}

Table D.9: Coverage of nominal 95\% GMM Intervals with $n=1000$ 


\begin{tabular}{rr|rrrrrrrr}
\hline \hline & & \multicolumn{1}{|c}{$\beta$} & & & & & \\
$\alpha_{0}$ & $\alpha_{1}$ & 0 & 0.25 & 0.5 & 0.75 & 1 & 1.5 & 2 & 3 \\
\hline 0.0 & 0.0 & 19.07 & 3.44 & 1.86 & 1.32 & 0.87 & 0.47 & 0.37 & 0.35 \\
& 0.1 & 17.52 & 3.47 & 1.92 & 1.41 & 1 & 0.61 & 0.51 & 0.46 \\
& 0.2 & 17.41 & 3.51 & 1.9 & 1.45 & 1.1 & 0.76 & 0.65 & 0.58 \\
& 0.3 & 18.23 & 3.34 & 1.92 & 1.48 & 1.24 & 0.91 & 0.79 & 0.7 \\
\hline 0.1 & 0.0 & 17.13 & 3.51 & 1.86 & 1.38 & 0.97 & 0.61 & 0.51 & 0.46 \\
& 0.1 & 17.88 & 3.33 & 1.85 & 1.45 & 1.13 & 0.78 & 0.67 & 0.6 \\
& 0.2 & 17.37 & 3.36 & 1.95 & 1.54 & 1.24 & 0.97 & 0.85 & 0.75 \\
& 0.3 & 18.07 & 3.33 & 1.98 & 1.63 & 1.41 & 1.17 & 1.04 & 0.92 \\
\hline 0.2 & 0.0 & 17.79 & 3.39 & 1.92 & 1.45 & 1.11 & 0.75 & 0.65 & 0.58 \\
& 0.1 & 18.98 & 3.43 & 1.96 & 1.54 & 1.26 & 0.97 & 0.84 & 0.75 \\
& 0.2 & 18.25 & 3.26 & 1.92 & 1.64 & 1.45 & 1.2 & 1.06 & 0.95 \\
& 0.3 & 19.03 & 3.31 & 2.02 & 1.75 & 1.66 & 1.49 & 1.33 & 1.19 \\
\hline 0.3 & 0.0 & 18.27 & 3.48 & 1.87 & 1.5 & 1.25 & 0.9 & 0.79 & 0.7 \\
& 0.1 & 19.4 & 3.41 & 1.96 & 1.63 & 1.43 & 1.18 & 1.04 & 0.92 \\
& 0.2 & 18.22 & 3.56 & 1.96 & 1.74 & 1.67 & 1.49 & 1.35 & 1.19 \\
& 0.3 & 17.56 & 3.55 & 2.13 & 1.96 & 1.86 & 1.86 & 1.74 & 1.55 \\
\hline
\end{tabular}

Table D.10: Median Width of nominal 95\% GMM Intervals with $n=1000$

\begin{tabular}{rr|rrrrrrrr}
\hline \hline & & \multicolumn{1}{|c}{$\beta$} & & & & & \\
$\alpha_{0}$ & $\alpha_{1}$ & 0 & 0.25 & 0.5 & 0.75 & 1 & 1.5 & 2 & 3 \\
\hline 0.0 & 0.0 & 74 & 54 & 63 & 87 & 95 & 94 & 96 & 95 \\
& 0.1 & 72 & 54 & 62 & 86 & 94 & 95 & 95 & 96 \\
& 0.2 & 72 & 53 & 64 & 85 & 94 & 95 & 95 & 94 \\
& 0.3 & 73 & 54 & 64 & 81 & 94 & 95 & 95 & 94 \\
\hline 0.1 & 0.0 & 73 & 54 & 65 & 83 & 94 & 95 & 94 & 96 \\
& 0.1 & 74 & 55 & 64 & 84 & 93 & 95 & 95 & 95 \\
& 0.2 & 72 & 52 & 63 & 80 & 93 & 96 & 95 & 95 \\
& 0.3 & 75 & 53 & 59 & 77 & 90 & 95 & 95 & 95 \\
\hline 0.2 & 0.0 & 74 & 54 & 61 & 84 & 93 & 96 & 95 & 94 \\
& 0.1 & 74 & 54 & 63 & 81 & 92 & 96 & 95 & 96 \\
& 0.2 & 73 & 52 & 60 & 75 & 90 & 96 & 96 & 95 \\
& 0.3 & 74 & 50 & 57 & 72 & 86 & 95 & 96 & 96 \\
\hline 0.3 & 0.0 & 74 & 53 & 61 & 83 & 92 & 97 & 95 & 95 \\
& 0.1 & 75 & 52 & 60 & 78 & 90 & 95 & 96 & 96 \\
& 0.2 & 73 & 52 & 57 & 73 & 85 & 95 & 96 & 96 \\
& 0.3 & 73 & 53 & 54 & 69 & 80 & 93 & 96 & 96 \\
\hline
\end{tabular}

Table D.11: Coverage of nominal 95\% GMM Intervals with $n=2000$ 


\begin{tabular}{rr|rrrrrrrr}
\hline \hline & & \multicolumn{1}{|c}{$\beta$} & & & & & \\
$\alpha_{0}$ & $\alpha_{1}$ & 0 & 0.25 & 0.5 & 0.75 & 1 & 1.5 & 2 & 3 \\
\hline 0.0 & 0.0 & 17.4 & 2.42 & 1.47 & 1 & 0.62 & 0.33 & 0.27 & 0.24 \\
& 0.1 & 16.56 & 2.51 & 1.49 & 1.06 & 0.7 & 0.43 & 0.36 & 0.33 \\
& 0.2 & 16.33 & 2.4 & 1.53 & 1.13 & 0.81 & 0.53 & 0.46 & 0.41 \\
& 0.3 & 17.06 & 2.52 & 1.57 & 1.19 & 0.91 & 0.65 & 0.56 & 0.5 \\
\hline 0.1 & 0.0 & 17.2 & 2.5 & 1.53 & 1.05 & 0.71 & 0.43 & 0.36 & 0.33 \\
& 0.1 & 17.48 & 2.5 & 1.53 & 1.15 & 0.83 & 0.56 & 0.48 & 0.43 \\
& 0.2 & 16.32 & 2.45 & 1.57 & 1.2 & 0.97 & 0.69 & 0.6 & 0.53 \\
& 0.3 & 18.37 & 2.43 & 1.51 & 1.3 & 1.1 & 0.84 & 0.73 & 0.65 \\
\hline 0.2 & 0.0 & 17.64 & 2.5 & 1.49 & 1.13 & 0.8 & 0.54 & 0.46 & 0.41 \\
& 0.1 & 18.25 & 2.47 & 1.58 & 1.22 & 0.96 & 0.69 & 0.6 & 0.54 \\
& 0.2 & 17.02 & 2.4 & 1.57 & 1.31 & 1.13 & 0.86 & 0.76 & 0.67 \\
& 0.3 & 18.05 & 2.39 & 1.61 & 1.43 & 1.33 & 1.09 & 0.95 & 0.85 \\
\hline 0.3 & 0.0 & 17.72 & 2.43 & 1.53 & 1.19 & 0.91 & 0.65 & 0.56 & 0.5 \\
& 0.1 & 18.8 & 2.46 & 1.55 & 1.32 & 1.11 & 0.84 & 0.74 & 0.65 \\
& 0.2 & 18.24 & 2.45 & 1.61 & 1.45 & 1.3 & 1.08 & 0.96 & 0.85 \\
& 0.3 & 17.43 & 2.55 & 1.67 & 1.62 & 1.57 & 1.4 & 1.24 & 1.1 \\
\hline
\end{tabular}

Table D.12: Median Width of nominal 95\% GMM Intervals with $n=2000$

\begin{tabular}{rr|rrrrrrrr}
\hline \hline & & \multicolumn{8}{|c}{$\beta$} \\
$\alpha_{0}$ & $\alpha_{1}$ & 0 & 0.25 & 0.5 & 0.75 & 1 & 1.5 & 2 & 3 \\
\hline 0.0 & 0.0 & 96 & 97 & 97 & 96 & 97 & 97 & 95 & 96 \\
& 0.1 & 97 & 99 & 99 & 99 & 99 & 100 & 100 & 99 \\
& 0.2 & 98 & 99 & 99 & 100 & 100 & 100 & 100 & 100 \\
& 0.3 & 97 & 100 & 100 & 100 & 100 & 100 & 100 & 100 \\
\hline 0.1 & 0.0 & 97 & 99 & 99 & 99 & 100 & 100 & 100 & 98 \\
& 0.1 & 98 & 100 & 100 & 100 & 100 & 100 & 100 & 100 \\
& 0.2 & 98 & 100 & 100 & 100 & 100 & 100 & 100 & 100 \\
& 0.3 & 97 & 100 & 100 & 100 & 100 & 100 & 100 & 100 \\
\hline 0.2 & 0.0 & 97 & 99 & 99 & 100 & 100 & 100 & 100 & 100 \\
& 0.1 & 98 & 100 & 100 & 100 & 100 & 100 & 100 & 100 \\
& 0.2 & 98 & 100 & 100 & 100 & 100 & 100 & 100 & 100 \\
& 0.3 & 98 & 100 & 100 & 100 & 100 & 100 & 100 & 100 \\
\hline 0.3 & 0.0 & 97 & 99 & 100 & 100 & 100 & 100 & 100 & 100 \\
& 0.1 & 97 & 100 & 100 & 100 & 100 & 100 & 100 & 100 \\
& 0.2 & 98 & 100 & 100 & 100 & 100 & 100 & 100 & 100 \\
& 0.3 & 98 & 100 & 100 & 100 & 100 & 100 & 100 & 100 \\
\hline
\end{tabular}

Table D.13: Coverage of nominal $>95 \%$ Bonferroni Intervals with $n=1000$ 


\begin{tabular}{rr|rrrrrrrr}
\hline \hline & & \multicolumn{8}{|c}{$\beta$} \\
$\alpha_{0}$ & $\alpha_{1}$ & 0 & 0.25 & 0.5 & 0.75 & 1 & 1.5 & 2 & 3 \\
\hline 0.0 & 0.0 & 96 & 97 & 96 & 97 & 96 & 96 & 95 & 95 \\
& 0.1 & 97 & 98 & 99 & 100 & 100 & 100 & 100 & 99 \\
& 0.2 & 97 & 99 & 99 & 100 & 100 & 100 & 100 & 100 \\
& 0.3 & 97 & 99 & 100 & 100 & 100 & 100 & 100 & 100 \\
\hline 0.1 & 0.0 & 97 & 99 & 99 & 99 & 100 & 100 & 100 & 99 \\
& 0.1 & 98 & 100 & 100 & 100 & 100 & 100 & 100 & 100 \\
& 0.2 & 98 & 100 & 100 & 100 & 100 & 100 & 100 & 100 \\
& 0.3 & 98 & 100 & 100 & 100 & 100 & 100 & 100 & 100 \\
\hline 0.2 & 0.0 & 97 & 99 & 99 & 100 & 100 & 100 & 100 & 99 \\
& 0.1 & 98 & 100 & 100 & 100 & 100 & 100 & 100 & 100 \\
& 0.2 & 98 & 100 & 100 & 100 & 100 & 100 & 100 & 100 \\
& 0.3 & 98 & 100 & 100 & 100 & 100 & 100 & 100 & 100 \\
\hline 0.3 & 0.0 & 97 & 100 & 100 & 100 & 100 & 100 & 100 & 100 \\
& 0.1 & 97 & 100 & 100 & 100 & 100 & 100 & 100 & 100 \\
& 0.2 & 97 & 100 & 100 & 100 & 100 & 100 & 100 & 100 \\
& 0.3 & 97 & 100 & 100 & 100 & 100 & 100 & 100 & 100 \\
\hline
\end{tabular}

Table D.14: Coverage of nominal $>95 \%$ Bonferroni Intervals with $n=2000$

\begin{tabular}{rr|rrrrrrrr}
\hline \hline & & \multicolumn{1}{|c}{$\beta$} \\
$\alpha_{0}$ & $\alpha_{1}$ & 0 & 0.25 & 0.5 & 0.75 & 1 & 1.5 & 2 & 3 \\
\hline 0.0 & 0.0 & 0.4 & 0.41 & 0.43 & 0.43 & 0.43 & 0.42 & 0.41 & 0.41 \\
& 0.1 & 0.45 & 0.47 & 0.54 & 0.59 & 0.63 & 0.7 & 0.75 & 0.86 \\
& 0.2 & 0.51 & 0.54 & 0.65 & 0.76 & 0.85 & 0.95 & 1.01 & 1.17 \\
& 0.3 & 0.58 & 0.62 & 0.79 & 0.95 & 1.07 & 1.17 & 1.24 & 1.48 \\
\hline 0.1 & 0.0 & 0.45 & 0.47 & 0.54 & 0.59 & 0.63 & 0.7 & 0.76 & 0.88 \\
& 0.1 & 0.51 & 0.54 & 0.66 & 0.77 & 0.86 & 1.03 & 1.18 & 1.46 \\
& 0.2 & 0.58 & 0.63 & 0.8 & 0.98 & 1.12 & 1.38 & 1.55 & 1.88 \\
& 0.3 & 0.67 & 0.75 & 1 & 1.25 & 1.46 & 1.74 & 1.94 & 2.4 \\
\hline 0.2 & 0.0 & 0.51 & 0.54 & 0.65 & 0.76 & 0.86 & 0.96 & 1.02 & 1.19 \\
& 0.1 & 0.58 & 0.63 & 0.81 & 0.99 & 1.14 & 1.42 & 1.64 & 2.08 \\
& 0.2 & 0.67 & 0.75 & 1.01 & 1.29 & 1.54 & 1.97 & 2.33 & 2.9 \\
& 0.3 & 0.81 & 0.91 & 1.3 & 1.7 & 2.09 & 2.73 & 3.13 & 3.9 \\
\hline 0.3 & 0.0 & 0.58 & 0.62 & 0.8 & 0.95 & 1.09 & 1.18 & 1.25 & 1.5 \\
& 0.1 & 0.68 & 0.74 & 1.01 & 1.26 & 1.49 & 1.84 & 2.13 & 2.78 \\
& 0.2 & 0.81 & 0.91 & 1.3 & 1.7 & 2.11 & 2.8 & 3.4 & 4.48 \\
& 0.3 & 1.01 & 1.16 & 1.74 & 2.35 & 2.93 & 4.17 & 5.2 & 6.85 \\
\hline
\end{tabular}

Table D.15: Median Width of nominal $>95 \%$ Bonferroni Intervals with $n=1000$ 


\begin{tabular}{rr|rrrrrrrr}
\hline \hline & & \multicolumn{1}{|c}{$\beta$} \\
$\alpha_{0}$ & $\alpha_{1}$ & 0 & 0.25 & 0.5 & 0.75 & 1 & 1.5 & 2 & 3 \\
\hline 0.0 & 0.0 & 0.29 & 0.3 & 0.31 & 0.31 & 0.31 & 0.3 & 0.29 & 0.29 \\
& 0.1 & 0.32 & 0.35 & 0.4 & 0.44 & 0.48 & 0.53 & 0.55 & 0.61 \\
& 0.2 & 0.36 & 0.41 & 0.51 & 0.59 & 0.65 & 0.67 & 0.69 & 0.81 \\
& 0.3 & 0.41 & 0.48 & 0.64 & 0.76 & 0.81 & 0.8 & 0.85 & 1.01 \\
\hline 0.1 & 0.0 & 0.32 & 0.35 & 0.4 & 0.44 & 0.48 & 0.53 & 0.56 & 0.62 \\
& 0.1 & 0.36 & 0.41 & 0.51 & 0.6 & 0.69 & 0.82 & 0.88 & 1.02 \\
& 0.2 & 0.41 & 0.48 & 0.64 & 0.79 & 0.91 & 1.04 & 1.08 & 1.27 \\
& 0.3 & 0.48 & 0.59 & 0.82 & 1.02 & 1.16 & 1.25 & 1.33 & 1.61 \\
\hline 0.2 & 0.0 & 0.36 & 0.41 & 0.51 & 0.59 & 0.65 & 0.67 & 0.7 & 0.82 \\
& 0.1 & 0.41 & 0.48 & 0.65 & 0.79 & 0.92 & 1.09 & 1.21 & 1.52 \\
& 0.2 & 0.48 & 0.59 & 0.83 & 1.05 & 1.24 & 1.49 & 1.61 & 1.96 \\
& 0.3 & 0.57 & 0.73 & 1.09 & 1.43 & 1.69 & 1.9 & 2.08 & 2.6 \\
\hline 0.3 & 0.0 & 0.41 & 0.48 & 0.64 & 0.77 & 0.82 & 0.78 & 0.84 & 1.02 \\
& 0.1 & 0.48 & 0.59 & 0.83 & 1.03 & 1.18 & 1.36 & 1.57 & 2.06 \\
& 0.2 & 0.57 & 0.73 & 1.1 & 1.43 & 1.71 & 2.11 & 2.45 & 3.18 \\
& 0.3 & 0.72 & 0.95 & 1.5 & 2.03 & 2.53 & 3.15 & 3.56 & 4.56 \\
\hline
\end{tabular}

Table D.16: Median Width of nominal $>95 \%$ Bonferroni Intervals with $n=2000$

\begin{tabular}{rr|rrrrrrrr}
\hline \hline & & \multicolumn{1}{|c}{$\beta$} & & & & & & \\
$\alpha_{0}$ & $\alpha_{1}$ & 0 & 0.25 & 0.5 & 0.75 & 1 & 1.5 & 2 & 3 \\
\hline 0.0 & 0.0 & 96 & 97 & 97 & 96 & 97 & 97 & 95 & 93 \\
& 0.1 & 97 & 99 & 99 & 99 & 99 & 98 & 96 & 95 \\
& 0.2 & 98 & 99 & 99 & 100 & 100 & 97 & 96 & 96 \\
& 0.3 & 97 & 100 & 100 & 100 & 99 & 96 & 96 & 96 \\
\hline 0.1 & 0.0 & 97 & 99 & 99 & 99 & 100 & 98 & 97 & 95 \\
& 0.1 & 98 & 100 & 100 & 100 & 100 & 96 & 96 & 96 \\
& 0.2 & 98 & 100 & 100 & 100 & 99 & 96 & 96 & 95 \\
& 0.3 & 97 & 100 & 100 & 100 & 97 & 95 & 96 & 96 \\
\hline 0.2 & 0.0 & 97 & 99 & 99 & 100 & 100 & 96 & 96 & 96 \\
& 0.1 & 98 & 100 & 100 & 100 & 99 & 96 & 96 & 96 \\
& 0.2 & 98 & 100 & 100 & 100 & 96 & 95 & 95 & 96 \\
& 0.3 & 98 & 100 & 100 & 98 & 95 & 95 & 95 & 96 \\
\hline 0.3 & 0.0 & 97 & 99 & 100 & 100 & 100 & 95 & 96 & 97 \\
& 0.1 & 97 & 100 & 100 & 100 & 97 & 94 & 96 & 96 \\
& 0.2 & 98 & 100 & 100 & 98 & 94 & 94 & 96 & 96 \\
& 0.3 & 98 & 100 & 99 & 96 & 92 & 94 & 95 & 96 \\
\hline
\end{tabular}

Table D.17: Coverage of hybrid CI constructed from nominal 95\% GMM and nominal > 95\% Bonferroni intervals: $n=1000$ 


\begin{tabular}{rr|rrrrrrrr}
\hline \hline & & \multicolumn{1}{|c}{$\beta$} \\
$\alpha_{0}$ & $\alpha_{1}$ & 0 & 0.25 & 0.5 & 0.75 & 1 & 1.5 & 2 & 3 \\
\hline 0.0 & 0.0 & 96 & 97 & 96 & 97 & 96 & 96 & 95 & 93 \\
& 0.1 & 97 & 98 & 99 & 100 & 100 & 98 & 97 & 96 \\
& 0.2 & 97 & 99 & 99 & 100 & 100 & 97 & 96 & 95 \\
& 0.3 & 97 & 99 & 100 & 100 & 99 & 96 & 96 & 96 \\
\hline 0.1 & 0.0 & 97 & 99 & 99 & 99 & 100 & 98 & 96 & 95 \\
& 0.1 & 98 & 100 & 100 & 100 & 100 & 96 & 96 & 97 \\
& 0.2 & 98 & 100 & 100 & 100 & 99 & 96 & 96 & 97 \\
& 0.3 & 98 & 100 & 100 & 99 & 97 & 95 & 96 & 96 \\
\hline 0.2 & 0.0 & 97 & 99 & 99 & 100 & 100 & 97 & 96 & 95 \\
& 0.1 & 98 & 100 & 100 & 100 & 98 & 96 & 96 & 97 \\
& 0.2 & 98 & 100 & 100 & 100 & 96 & 96 & 96 & 96 \\
& 0.3 & 98 & 100 & 100 & 97 & 95 & 95 & 96 & 96 \\
\hline 0.3 & 0.0 & 97 & 100 & 100 & 100 & 99 & 98 & 97 & 96 \\
& 0.1 & 97 & 100 & 100 & 100 & 96 & 95 & 96 & 97 \\
& 0.2 & 97 & 100 & 100 & 97 & 94 & 96 & 96 & 97 \\
& 0.3 & 97 & 100 & 100 & 94 & 94 & 95 & 96 & 96 \\
\hline
\end{tabular}

Table D.18: Coverage of hybrig CI constructed from nominal 95\% GMM and nominal > 95\% Bonferroni intervals: $n=2000$

\begin{tabular}{rr|rrrrrrrr}
\hline \hline & & \multicolumn{10}{|c}{$\beta$} \\
$\alpha_{0}$ & $\alpha_{1}$ & 0 & 0.25 & 0.5 & 0.75 & 1 & 1.5 & 2 & 3 \\
\hline 0.0 & 0.0 & 0.4 & 0.41 & 0.43 & 0.43 & 0.43 & 0.42 & 0.4 & 0.35 \\
& 0.1 & 0.45 & 0.47 & 0.54 & 0.59 & 0.63 & 0.67 & 0.52 & 0.46 \\
& 0.2 & 0.51 & 0.54 & 0.65 & 0.76 & 0.84 & 0.82 & 0.65 & 0.58 \\
& 0.3 & 0.58 & 0.62 & 0.79 & 0.95 & 1.05 & 0.96 & 0.79 & 0.7 \\
\hline 0.1 & 0.0 & 0.45 & 0.47 & 0.54 & 0.59 & 0.63 & 0.67 & 0.51 & 0.46 \\
& 0.1 & 0.51 & 0.54 & 0.66 & 0.77 & 0.86 & 0.92 & 0.69 & 0.61 \\
& 0.2 & 0.58 & 0.63 & 0.8 & 0.97 & 1.11 & 1.17 & 0.87 & 0.75 \\
& 0.3 & 0.67 & 0.75 & 1 & 1.25 & 1.4 & 1.4 & 1.06 & 0.92 \\
\hline 0.2 & 0.0 & 0.51 & 0.54 & 0.65 & 0.76 & 0.85 & 0.83 & 0.65 & 0.58 \\
& 0.1 & 0.58 & 0.63 & 0.81 & 0.99 & 1.12 & 1.18 & 0.86 & 0.75 \\
& 0.2 & 0.67 & 0.75 & 1.01 & 1.29 & 1.48 & 1.56 & 1.08 & 0.95 \\
& 0.3 & 0.81 & 0.91 & 1.3 & 1.67 & 1.95 & 1.77 & 1.35 & 1.2 \\
\hline 0.3 & 0.0 & 0.58 & 0.62 & 0.8 & 0.95 & 1.07 & 0.95 & 0.8 & 0.7 \\
& 0.1 & 0.68 & 0.74 & 1.01 & 1.26 & 1.43 & 1.48 & 1.06 & 0.93 \\
& 0.2 & 0.81 & 0.91 & 1.3 & 1.66 & 1.98 & 1.94 & 1.37 & 1.19 \\
& 0.3 & 1.01 & 1.16 & 1.73 & 2.24 & 2.71 & 2.33 & 1.78 & 1.55 \\
\hline
\end{tabular}

Table D.19: Median width of hybrid CI constructed from nominal 95\% GMM and nominal > 95\% Bonferroni intervals: $n=1000$ 

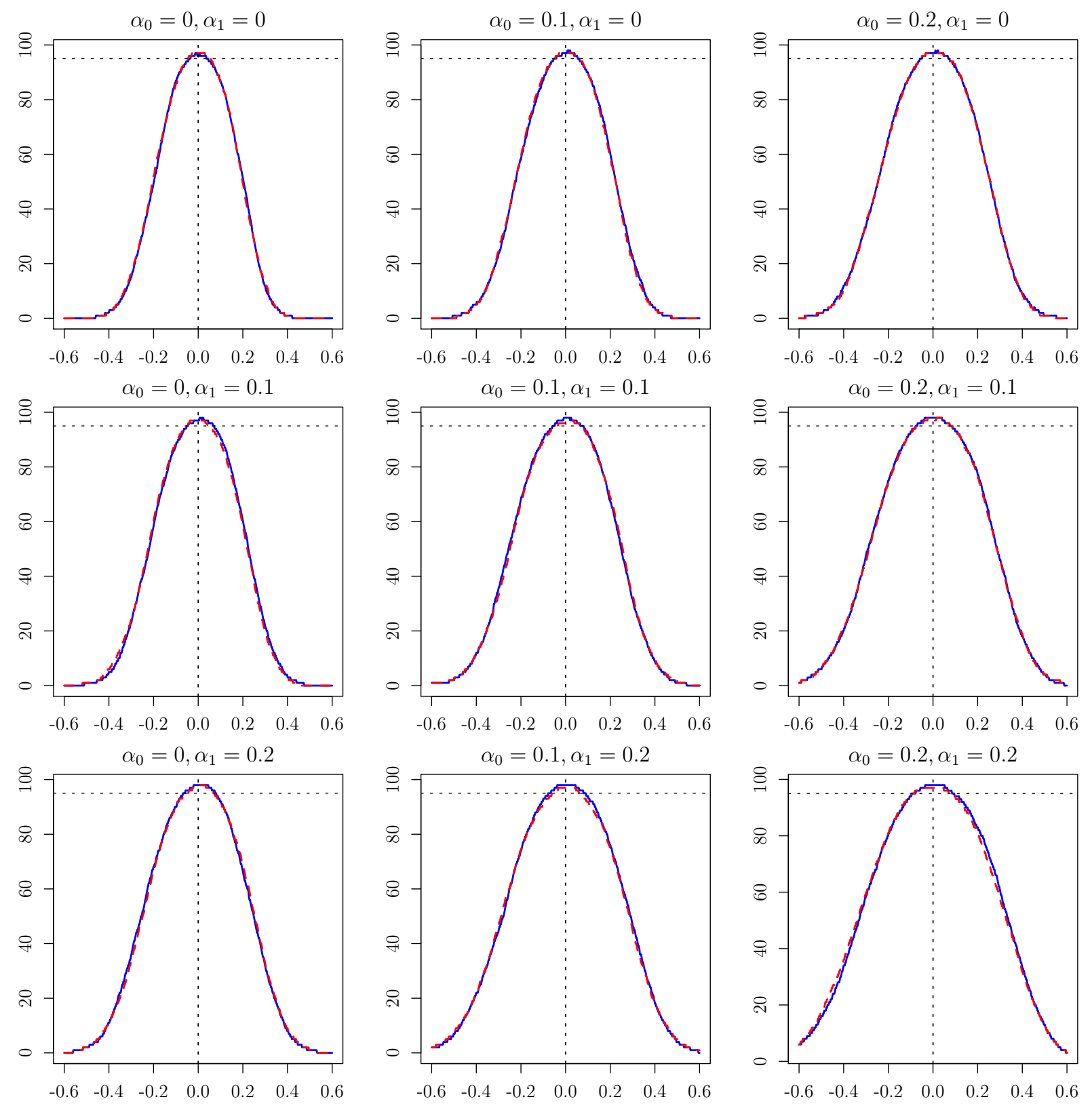

Figure D.1: Coverage Curves for Bonferroni with and without Non-differential Bounds: $\beta=$ $0, n=1000$ 

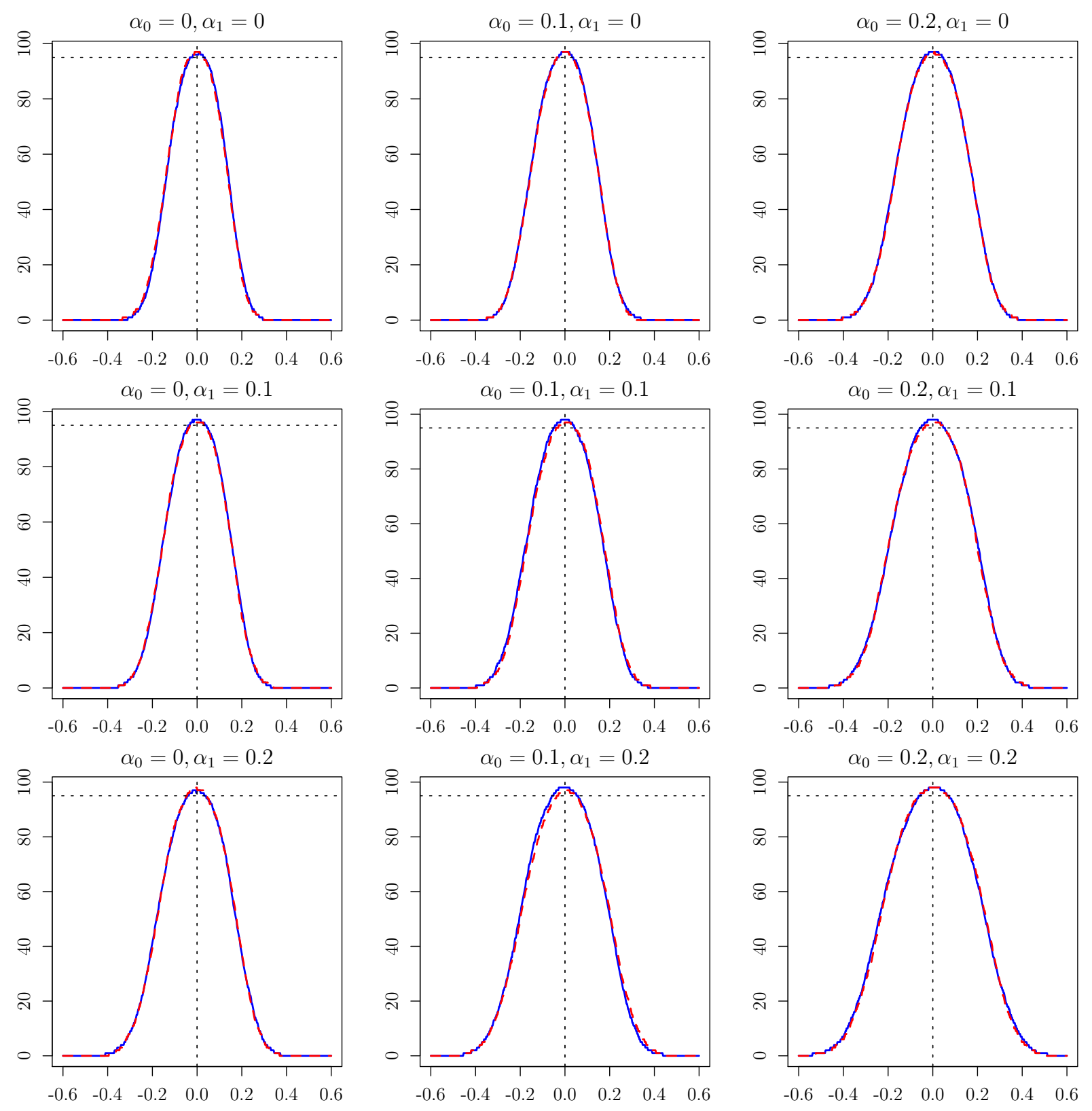

Figure D.2: Coverage Curves for Bonferroni with and without Non-differential Bounds: $\beta=$ $0, n=2000$ 

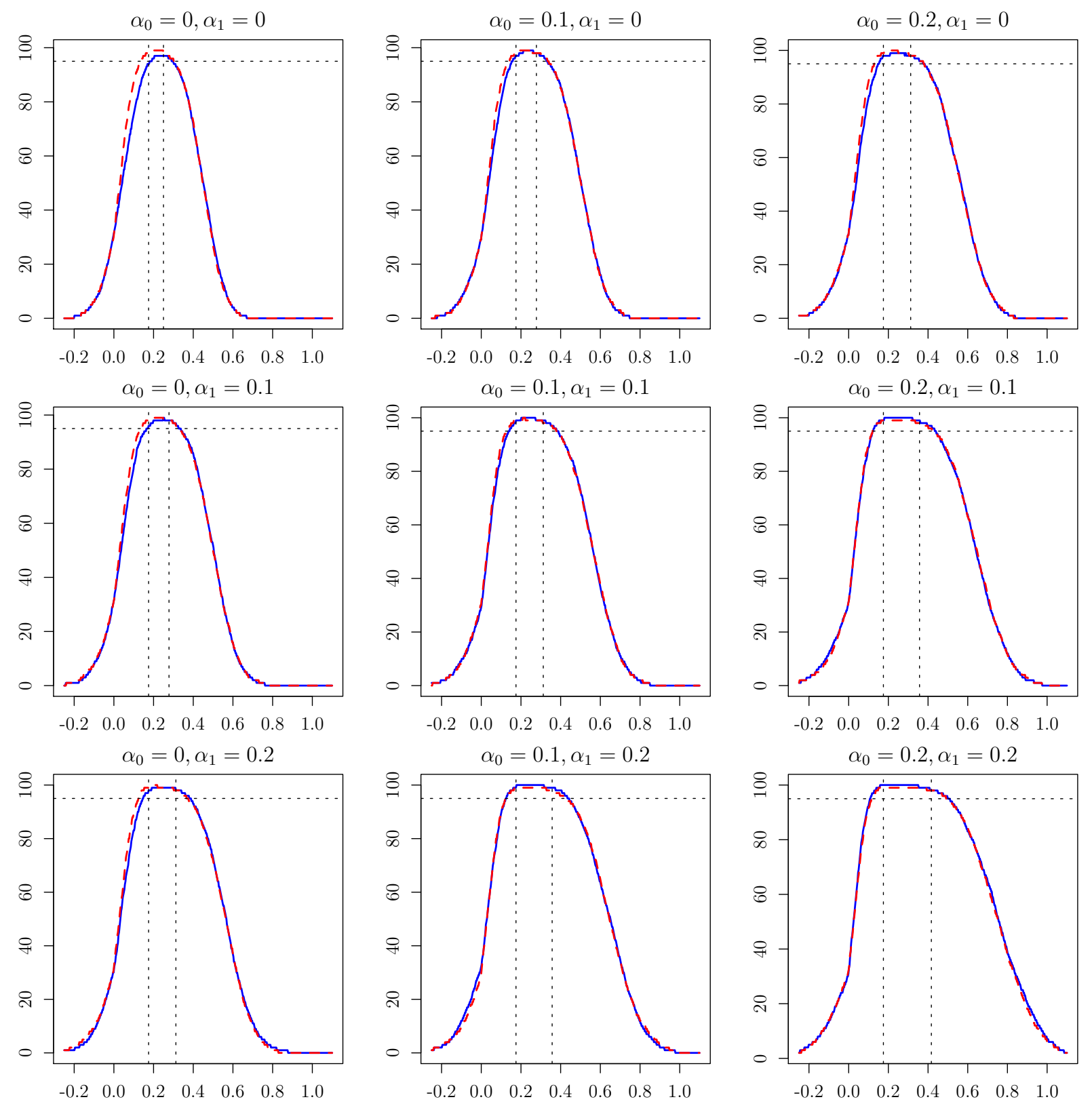

Figure D.3: Coverage Curves for Bonferroni with and without Non-differential Bounds: $\beta=$ $0.25, n=1000$ 

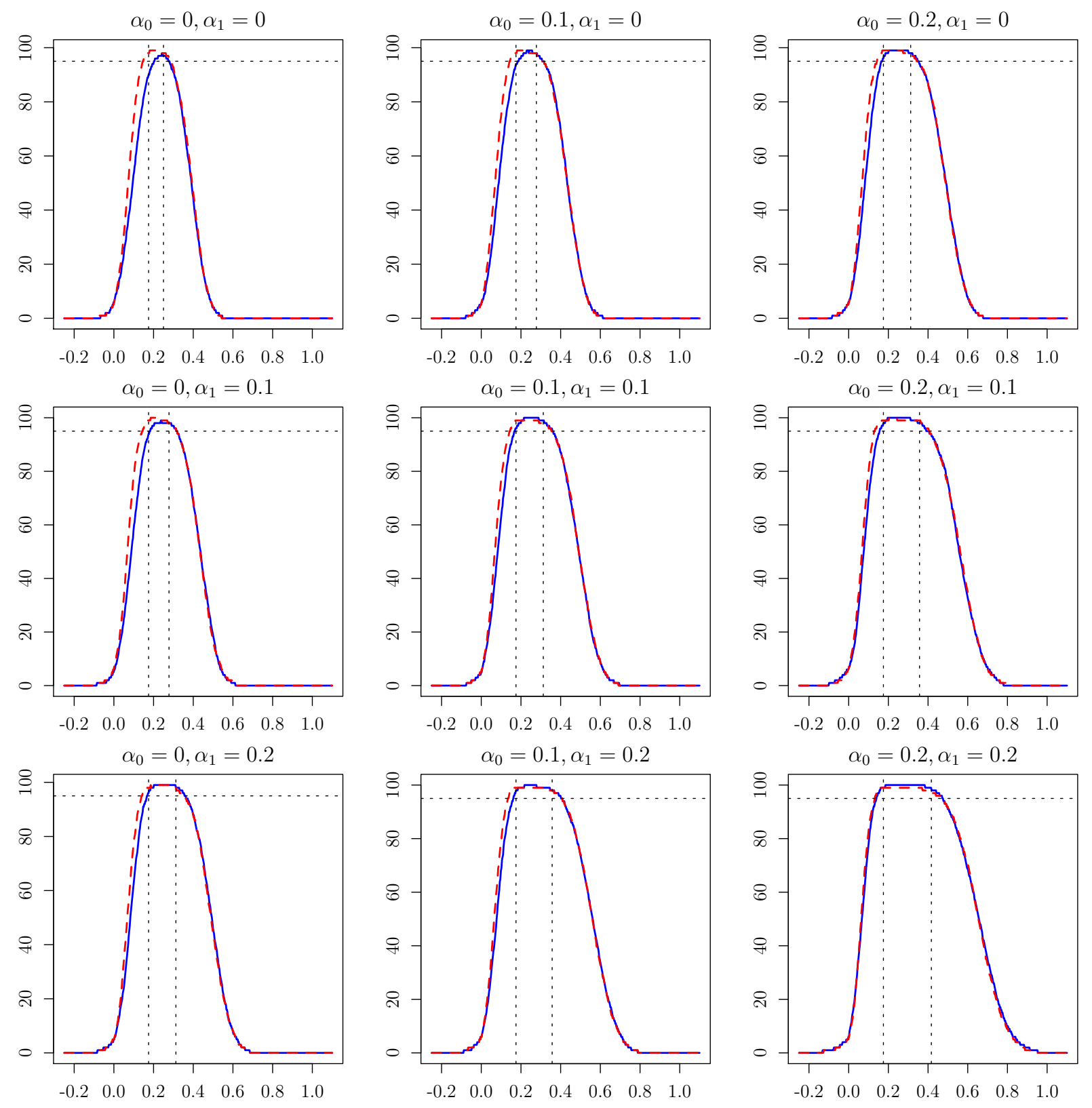

Figure D.4: Coverage Curves for Bonferroni with and without Non-differential Bounds: $\beta=$ $0.25, n=2000$ 

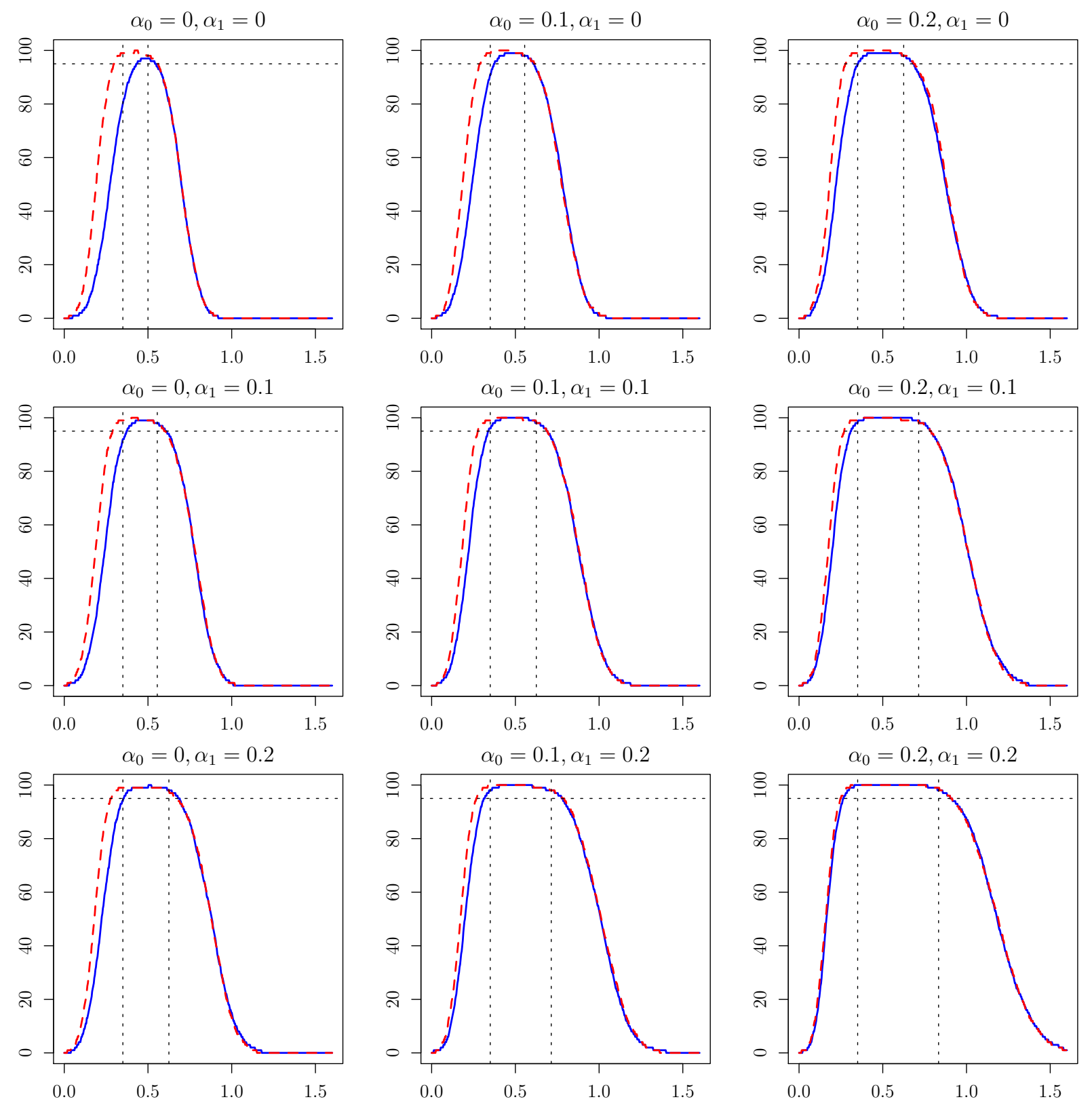

Figure D.5: Coverage Curves for Bonferroni with and without Non-differential Bounds: $\beta=$ $0.5, n=1000$ 

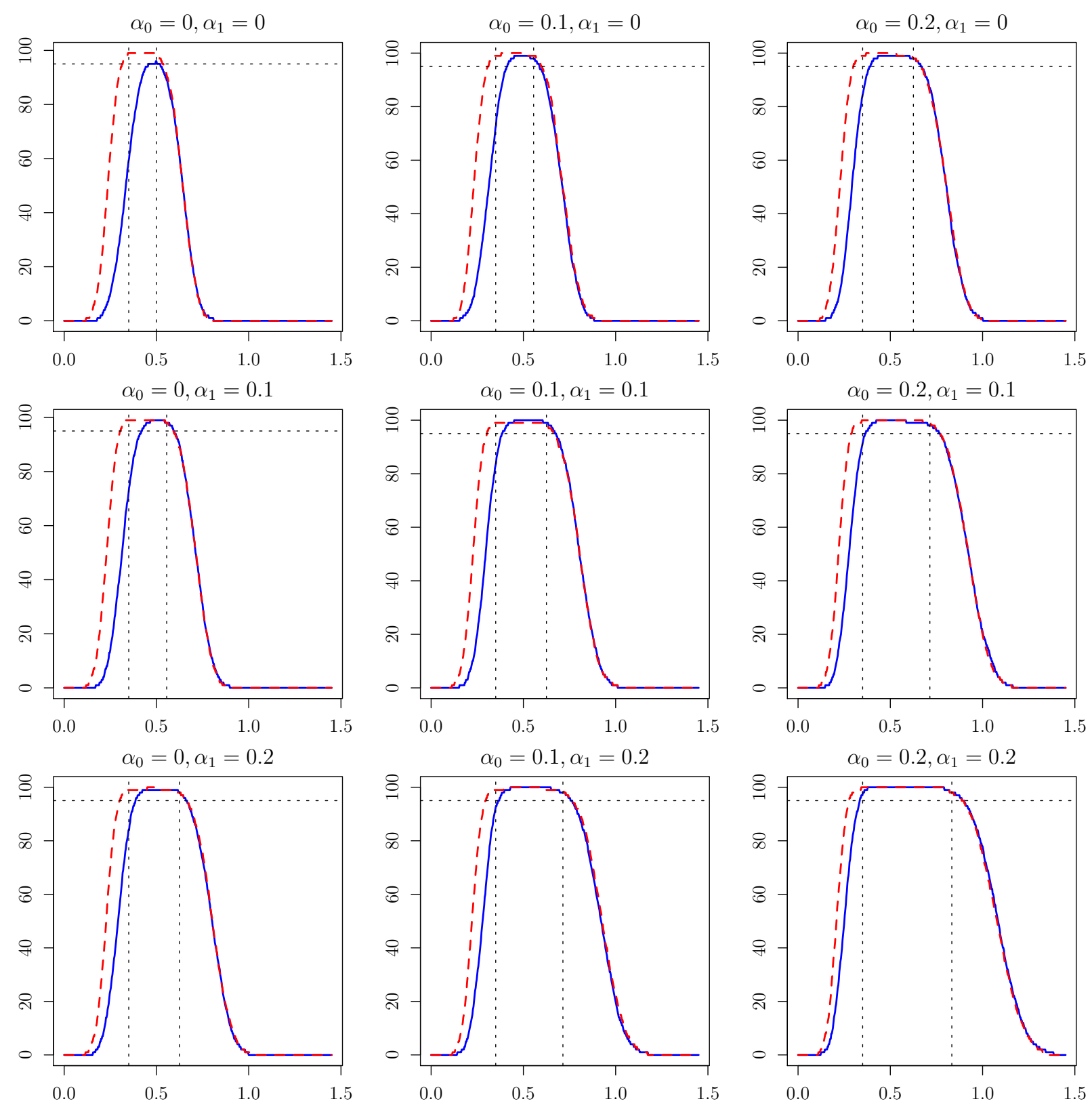

Figure D.6: Coverage Curves for Bonferroni with and without Non-differential Bounds: $\beta=$ $0.5, n=2000$ 

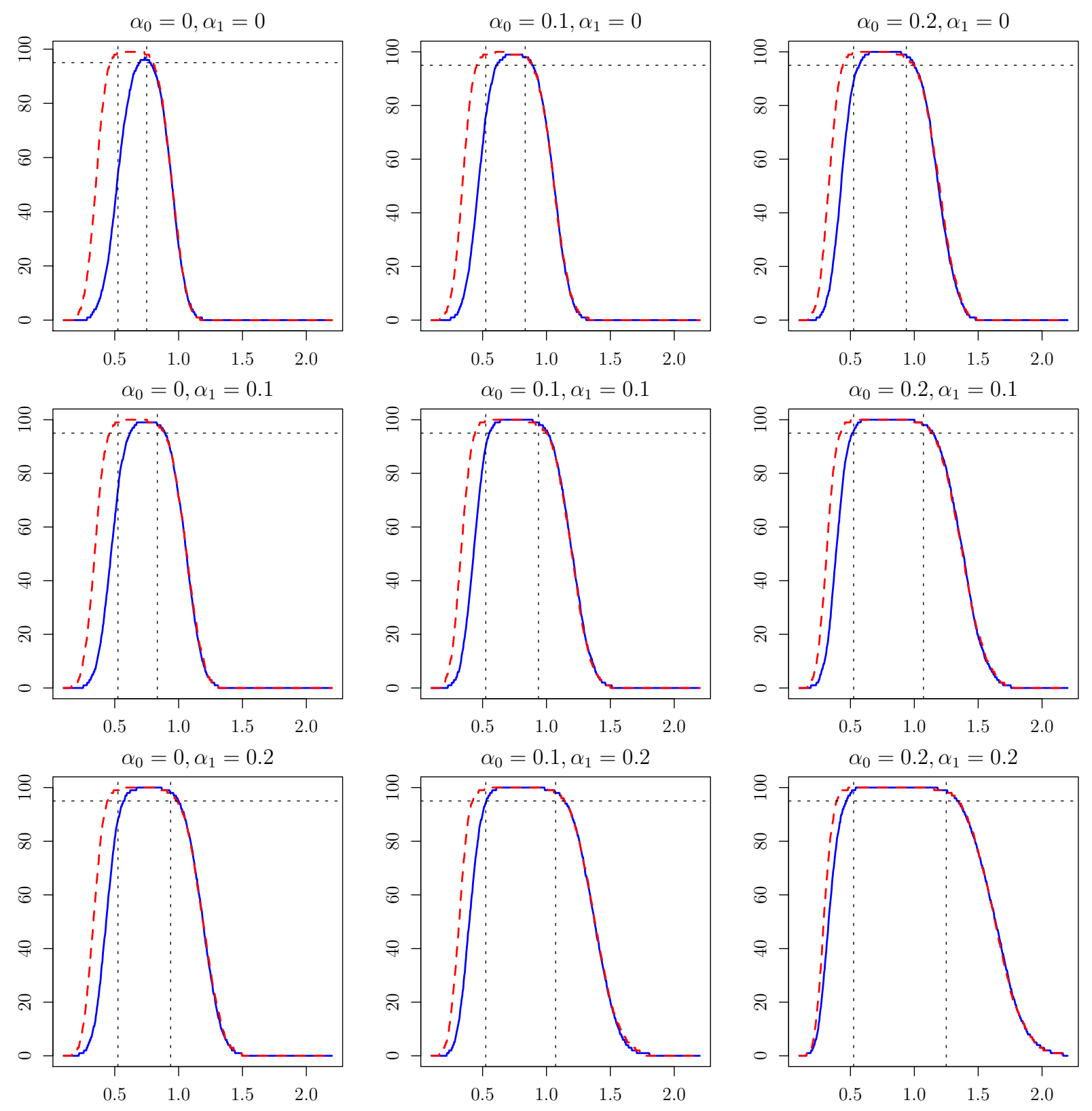

Figure D.7: Coverage Curves for Bonferroni with and without Non-differential Bounds: $\beta=$ $0.75, n=1000$ 

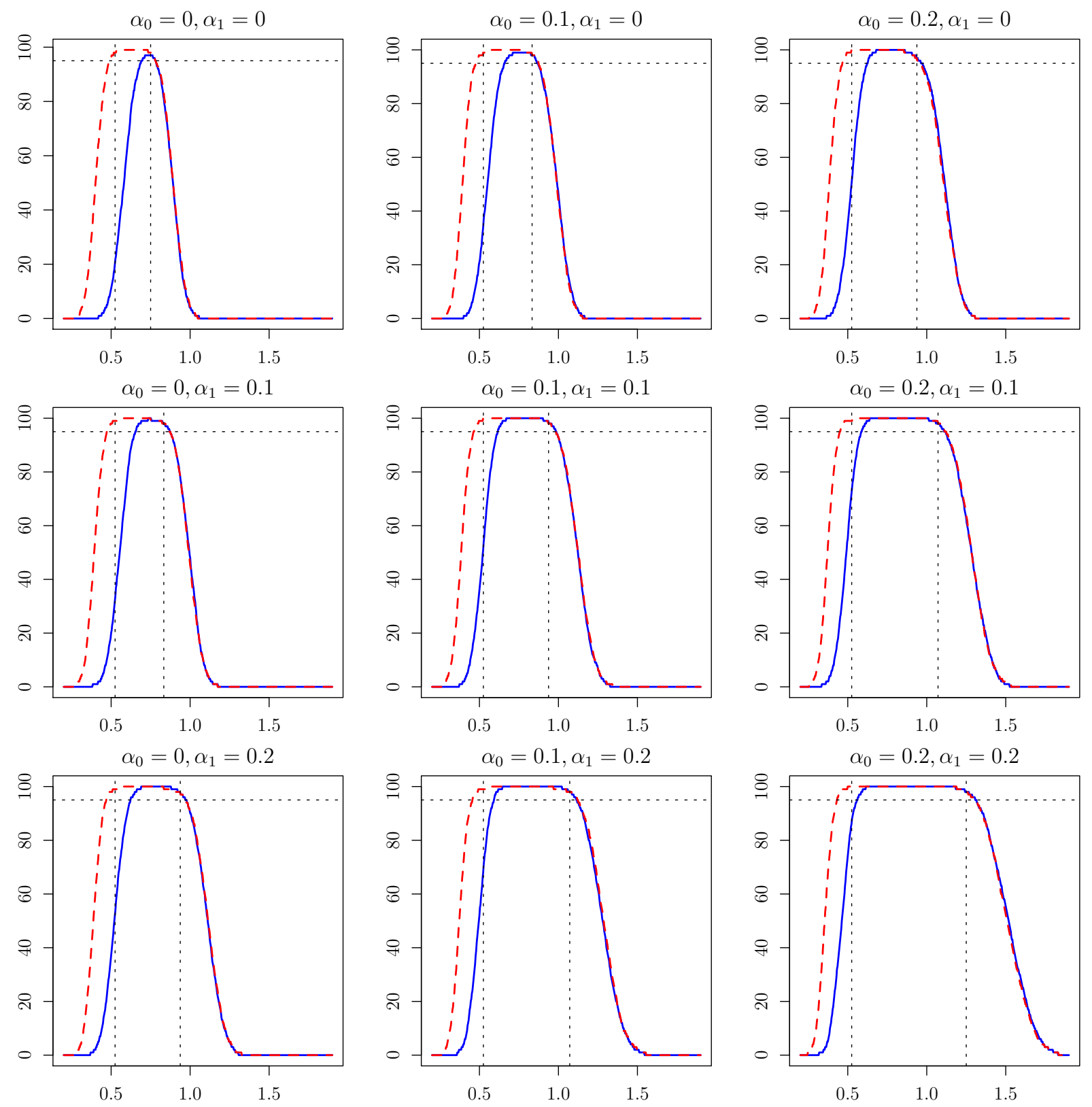

Figure D.8: Coverage Curves for Bonferroni with and without Non-differential Bounds: $\beta=$ $0.75, n=2000$ 

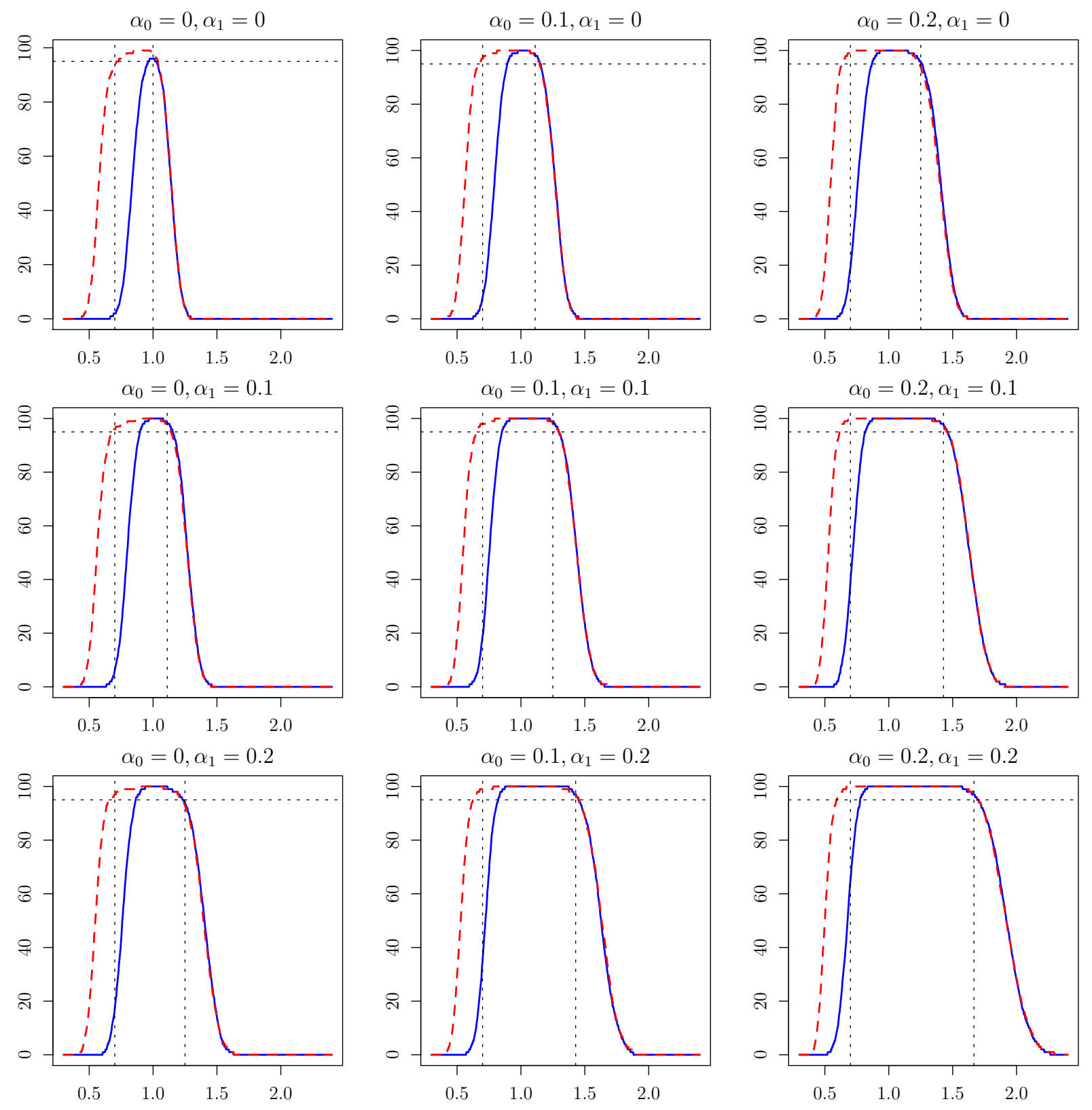

Figure D.9: Coverage Curves for Bonferroni with and without Non-differential Bounds: $\beta=$ $1, n=1000$ 

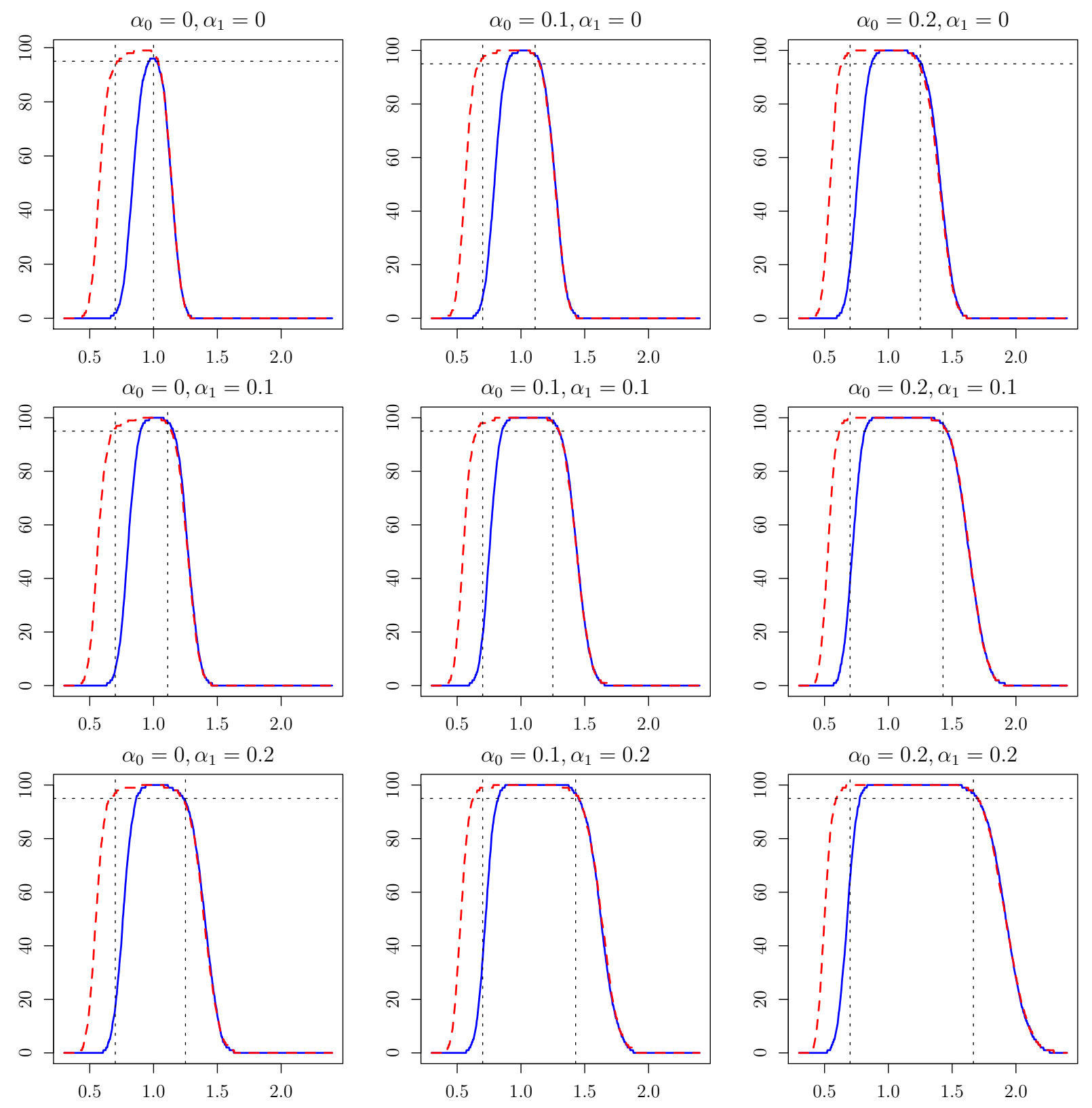

Figure D.10: Coverage Curves for Bonferroni with and without Non-differential Bounds: $\beta=$ $1, n=2000$ 

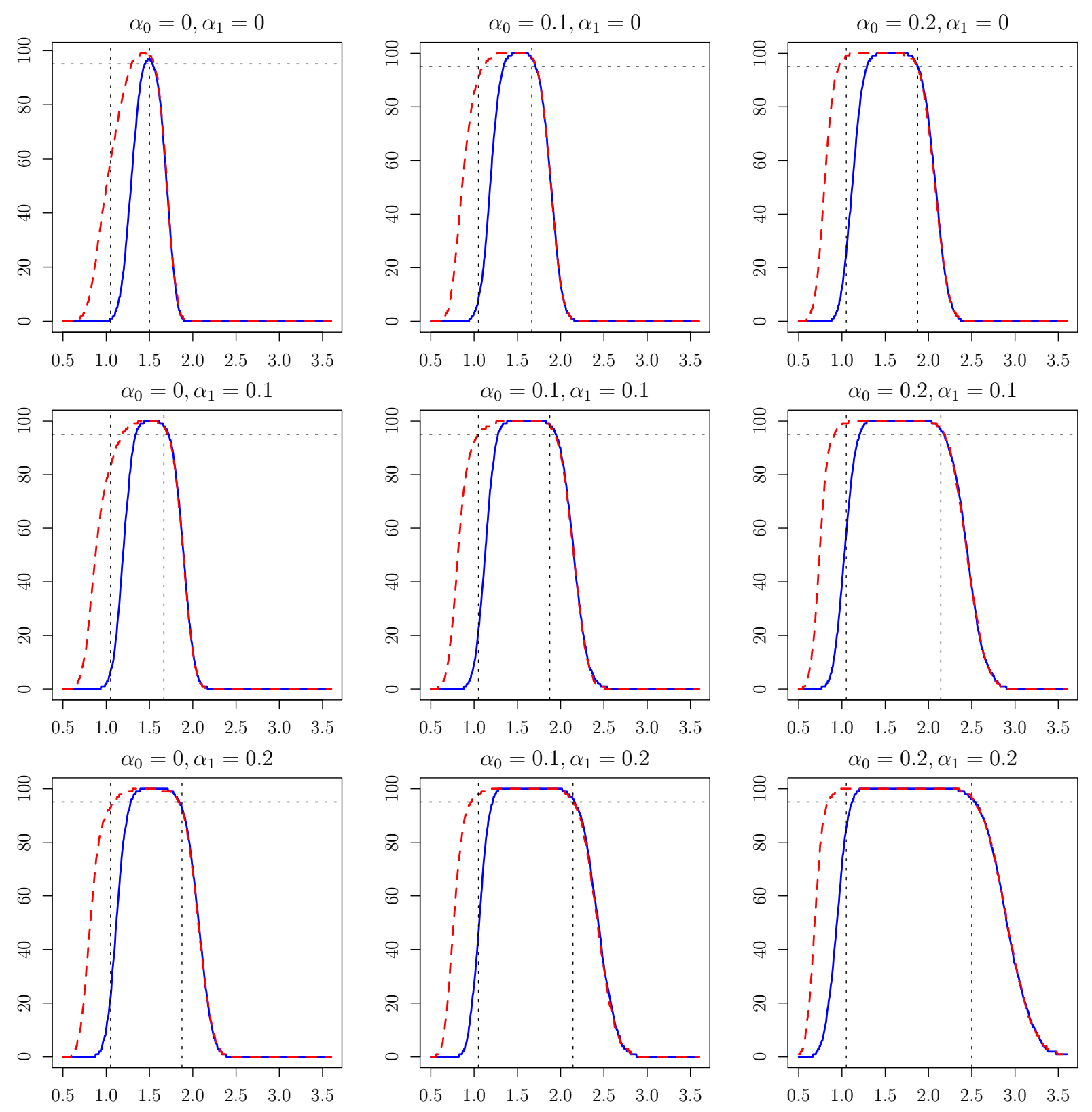

Figure D.11: Coverage Curves for Bonferroni with and without Non-differential Bounds: $\beta=$ $1.5, n=1000$ 

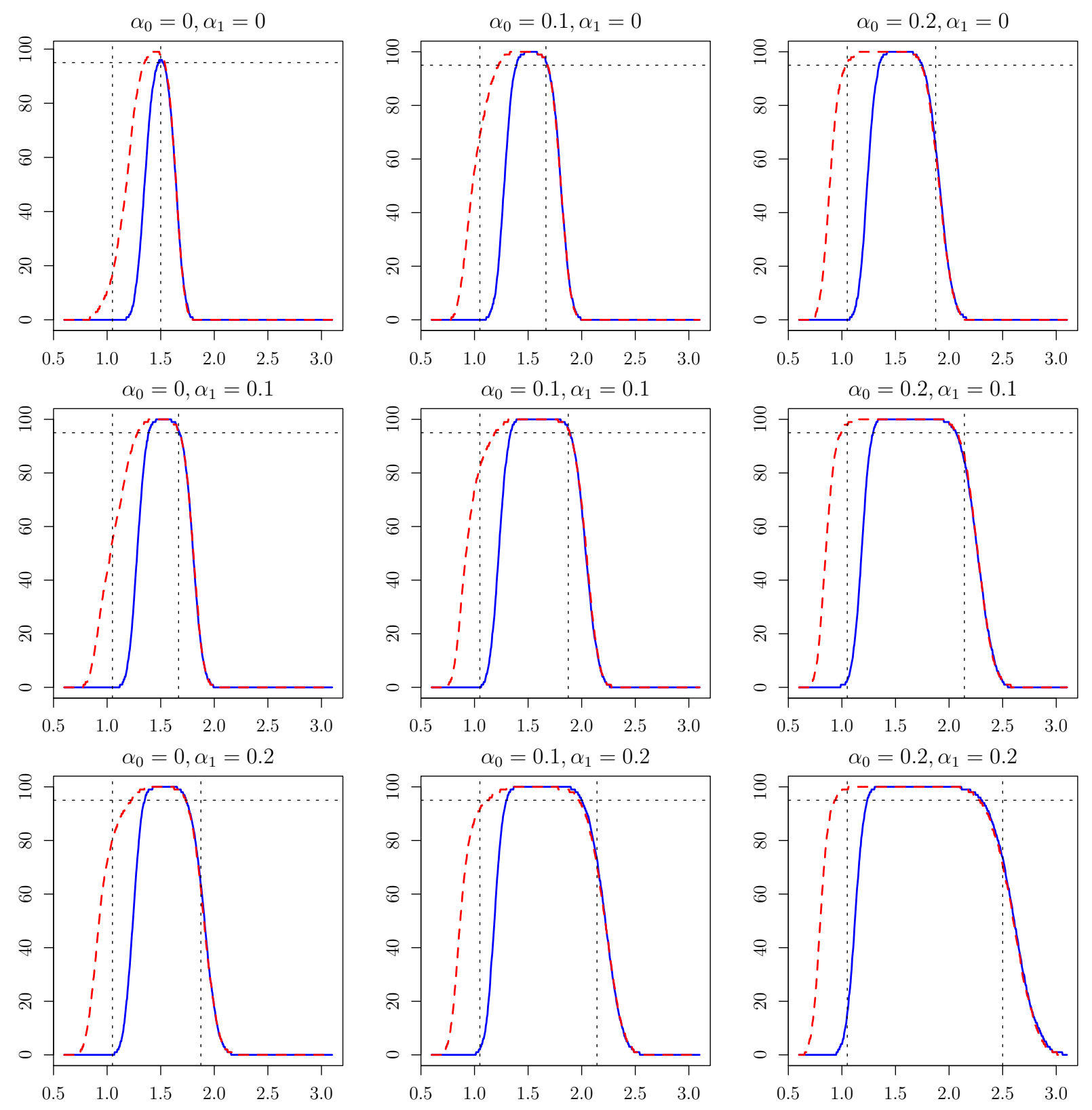

Figure D.12: Coverage Curves for Bonferroni with and without Non-differential Bounds: $\beta=$ $1.5, n=2000$ 

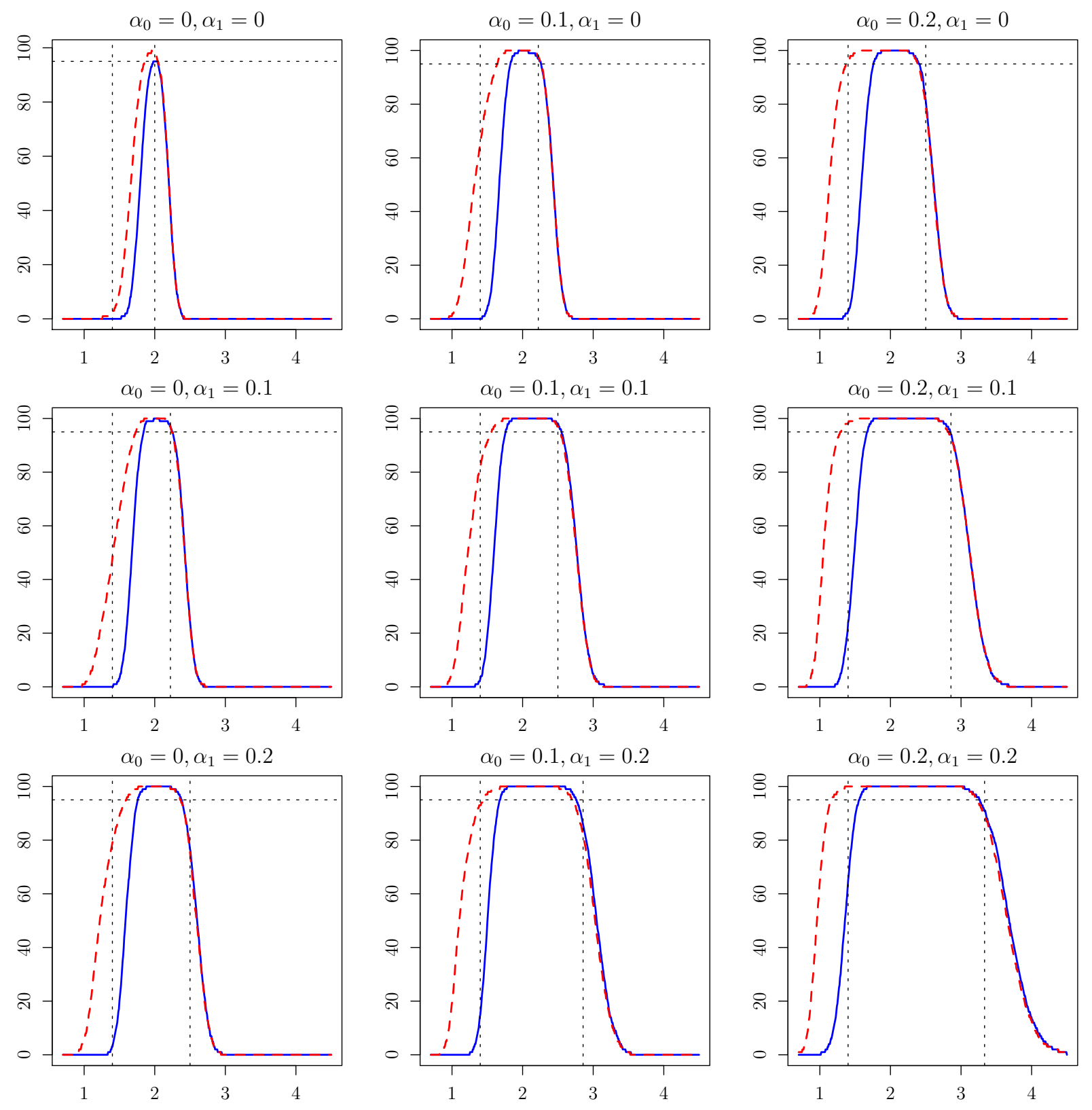

Figure D.13: Coverage Curves for Bonferroni with and without Non-differential Bounds: $\beta=$ $2, n=1000$ 

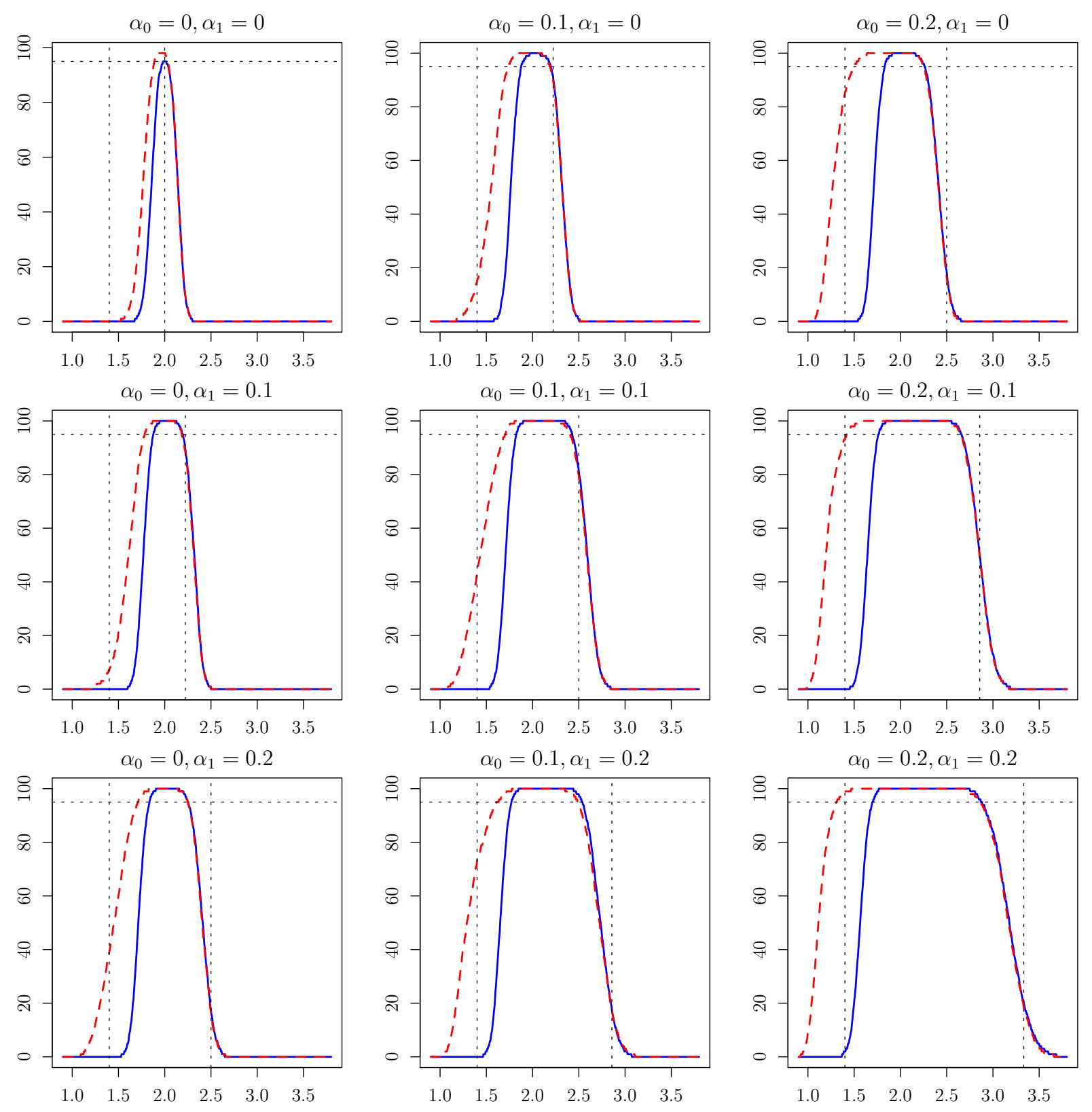

Figure D.14: Coverage Curves for Bonferroni with and without Non-differential Bounds: $\beta=$ $2, n=2000$ 

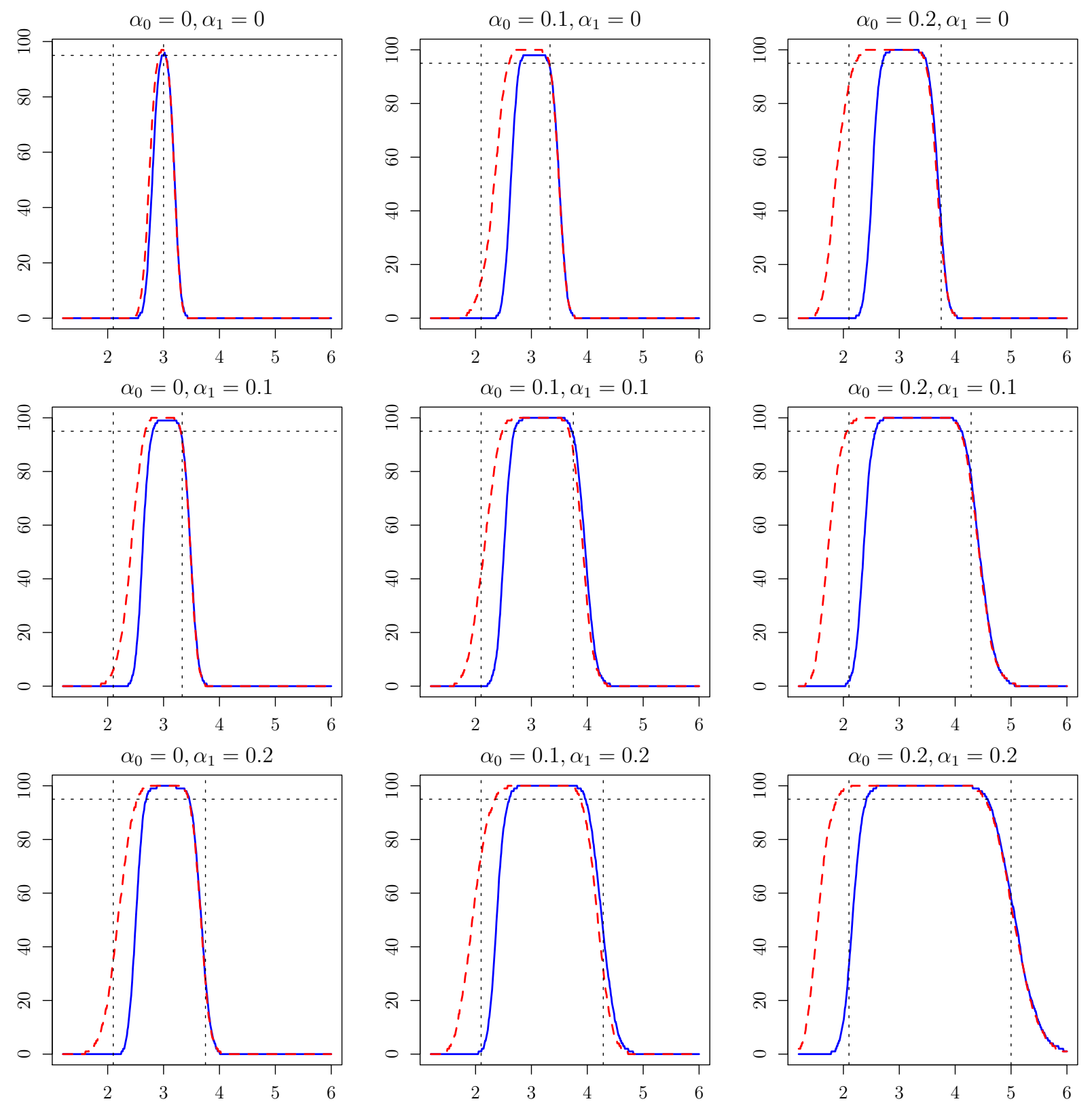

Figure D.15: Coverage Curves for Bonferroni with and without Non-differential Bounds: $\beta=$ $3, n=1000$ 

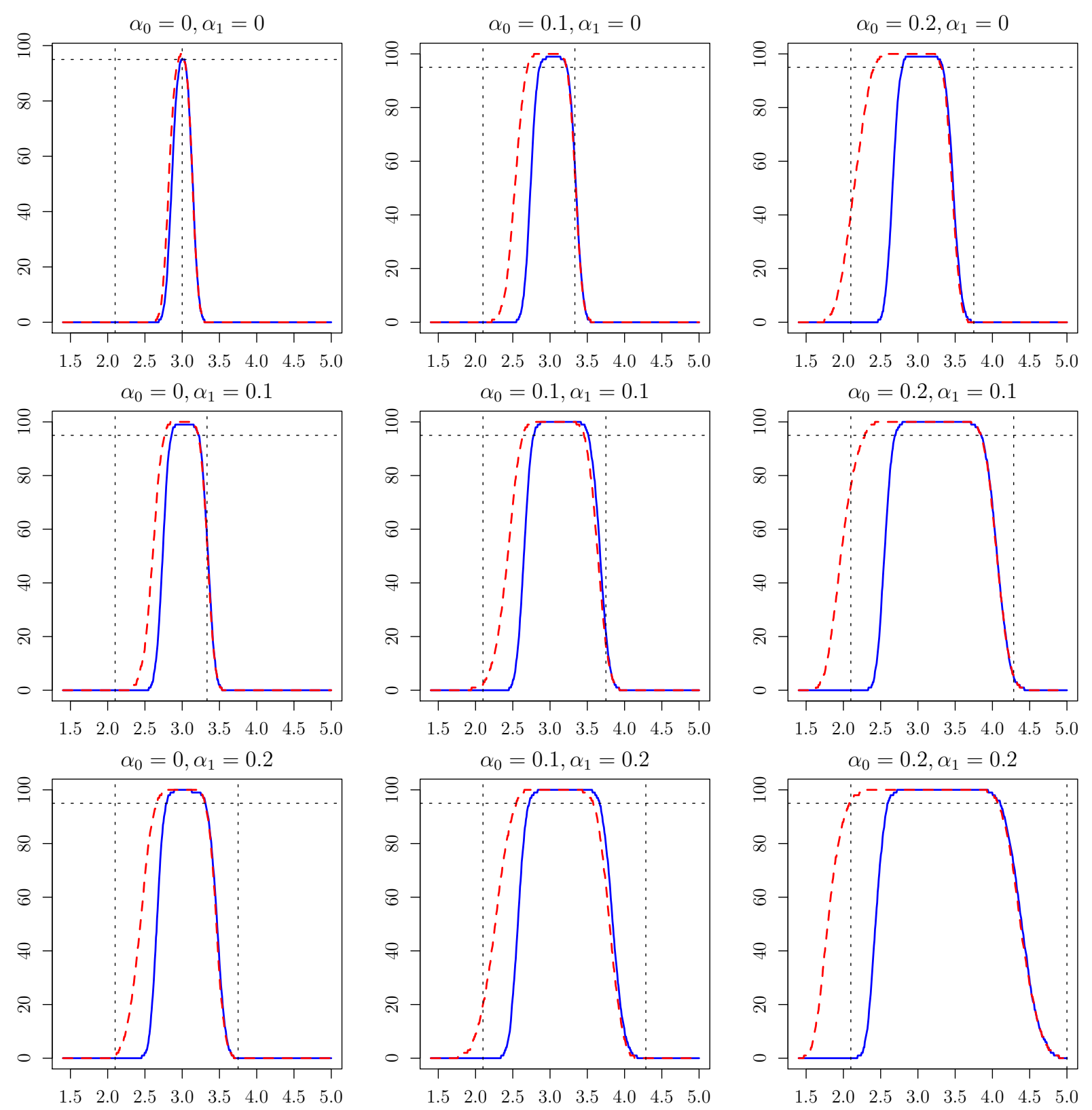

Figure D.16: Coverage Curves for Bonferroni with and without Non-differential Bounds: $\beta=$ $3, n=2000$ 

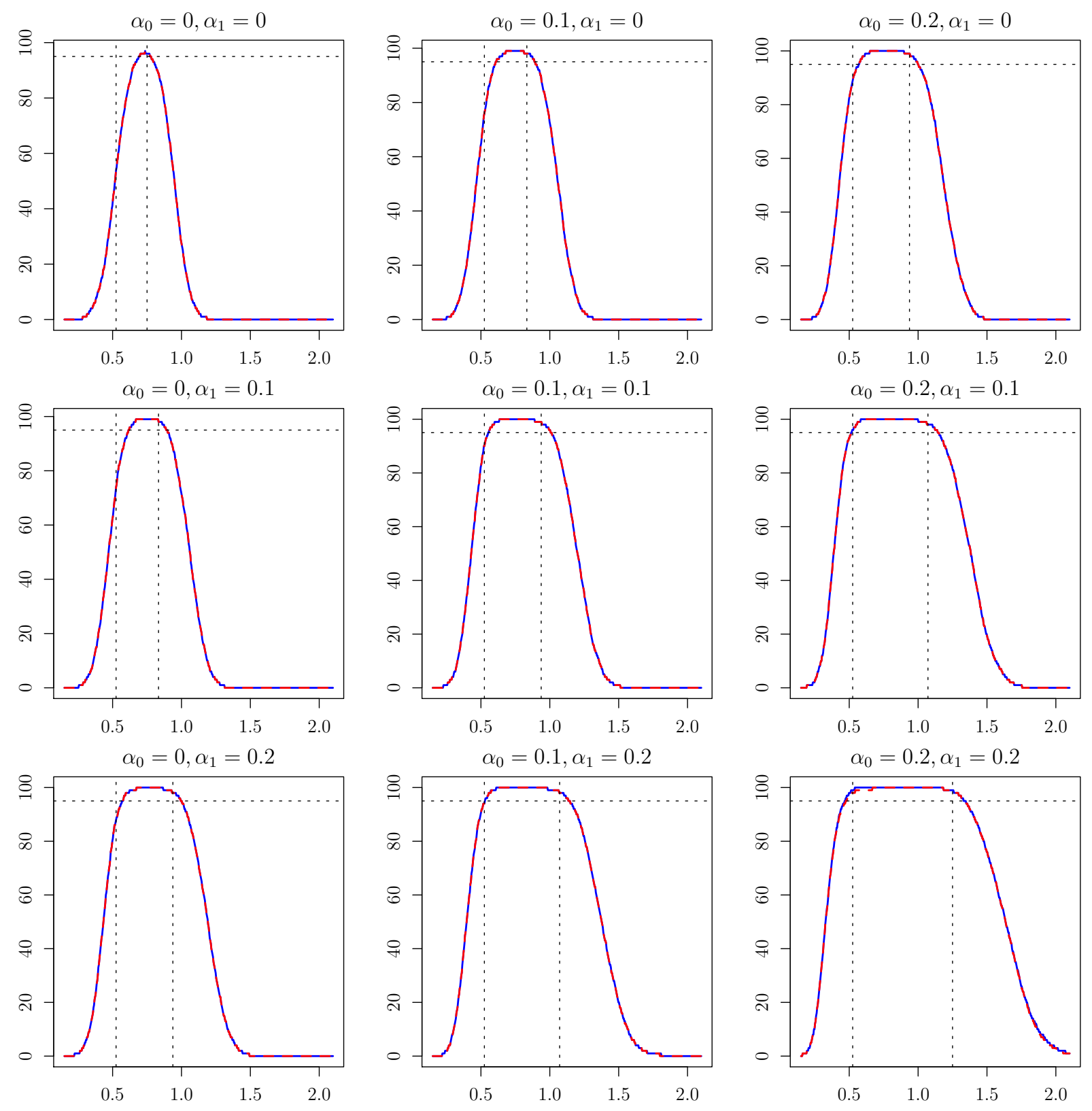

Figure D.17: Coverage Curves for Bonferroni versus Hybrid CIs: $\beta=0.75, n=1000$ 

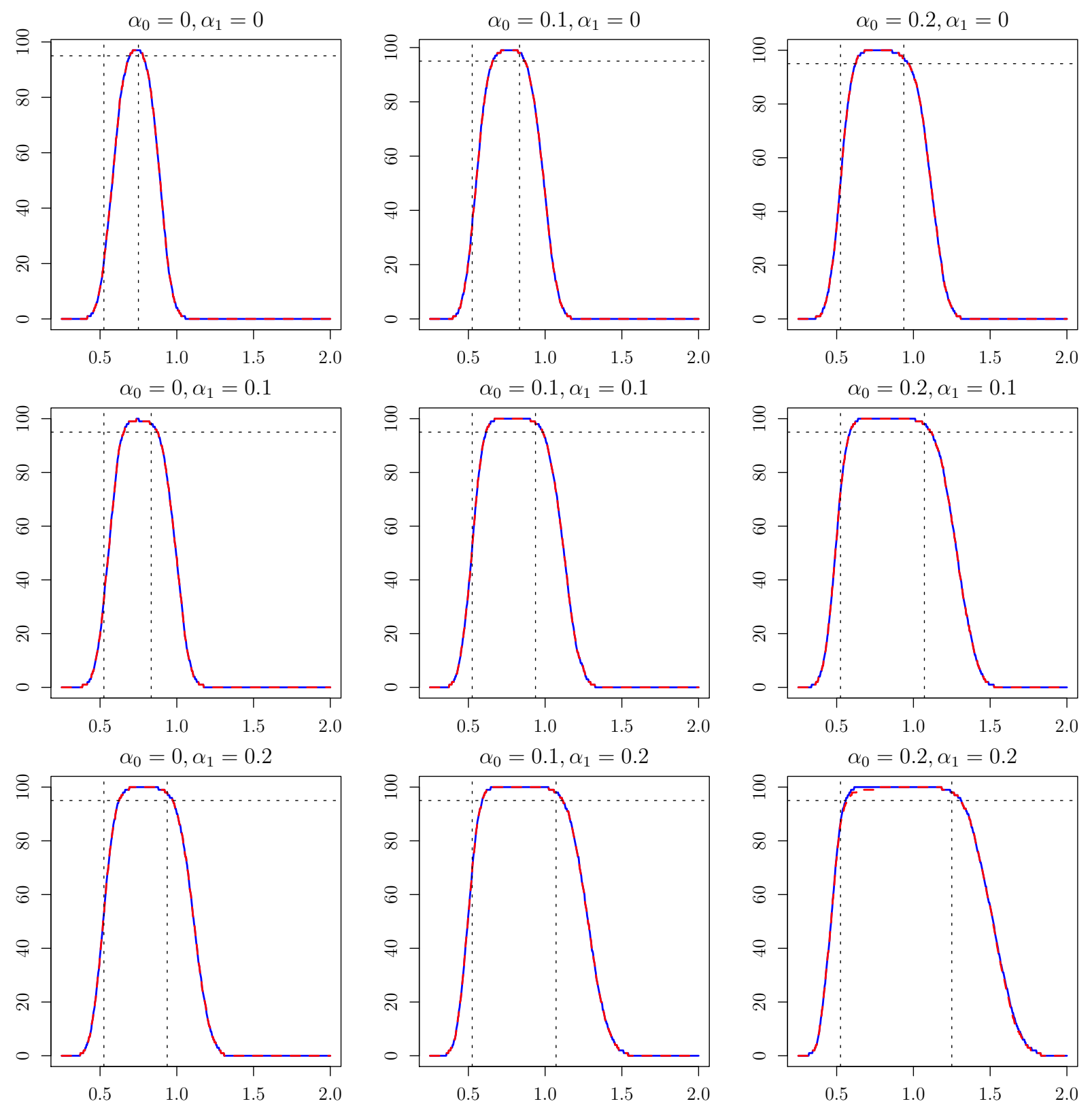

Figure D.18: Coverage Curves for Bonferroni versus Hybrid CIs: $\beta=0.75, n=2000$ 

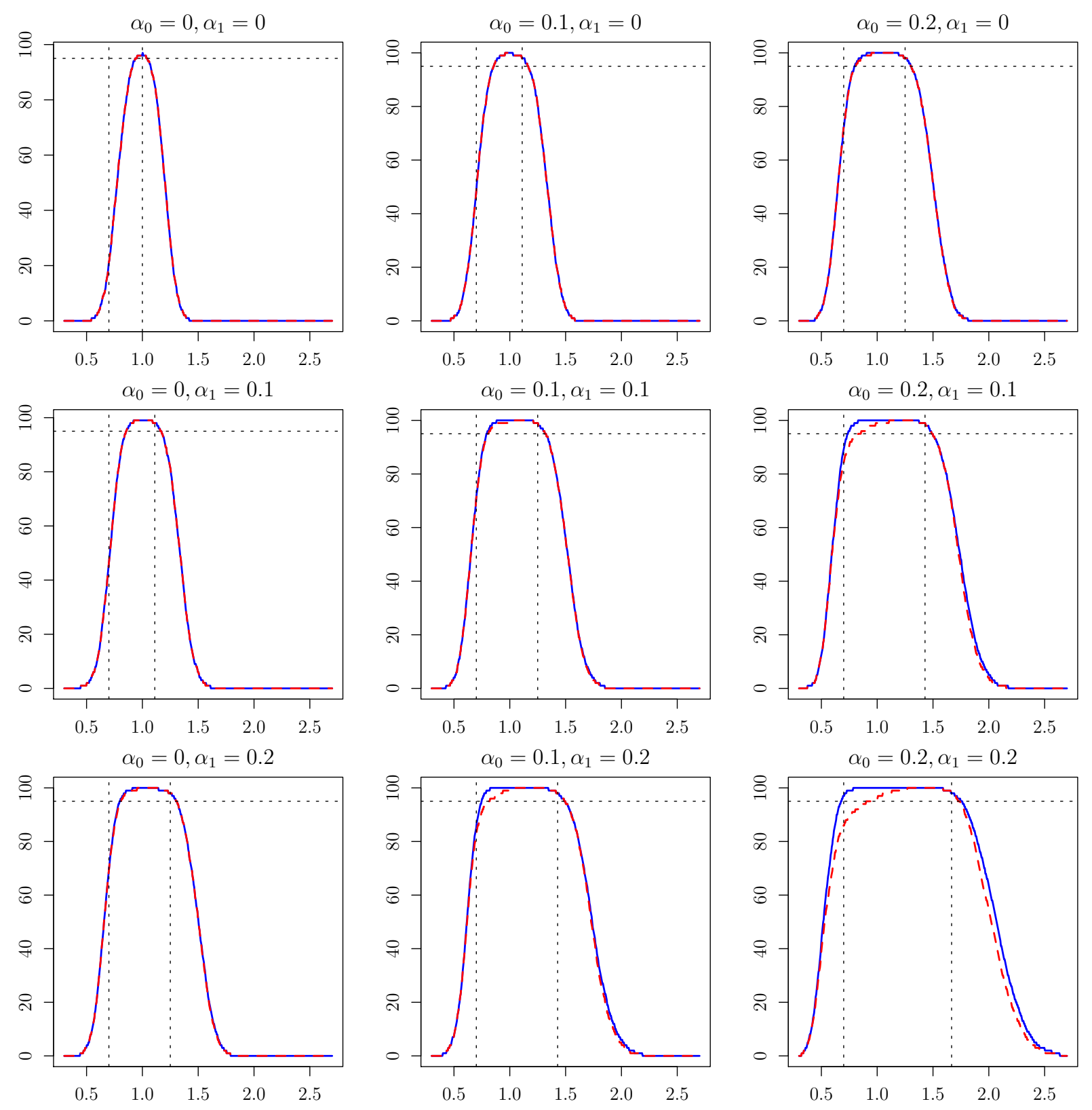

Figure D.19: Coverage Curves for Bonferroni versus Hybrid CIs: $\beta=1, n=1000$ 

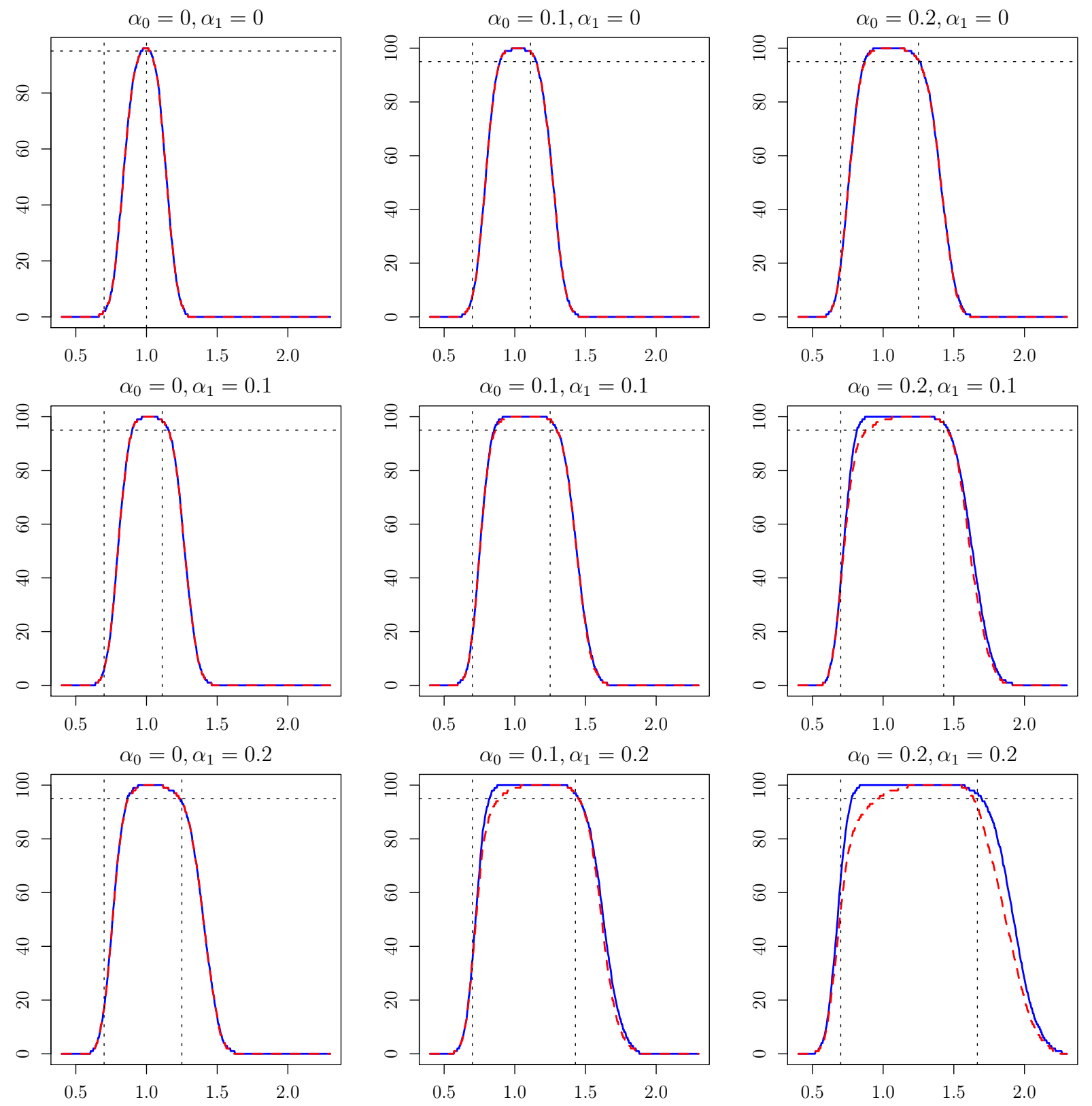

Figure D.20: Coverage Curves for Bonferroni versus Hybrid CIs: $\beta=1, n=2000$ 

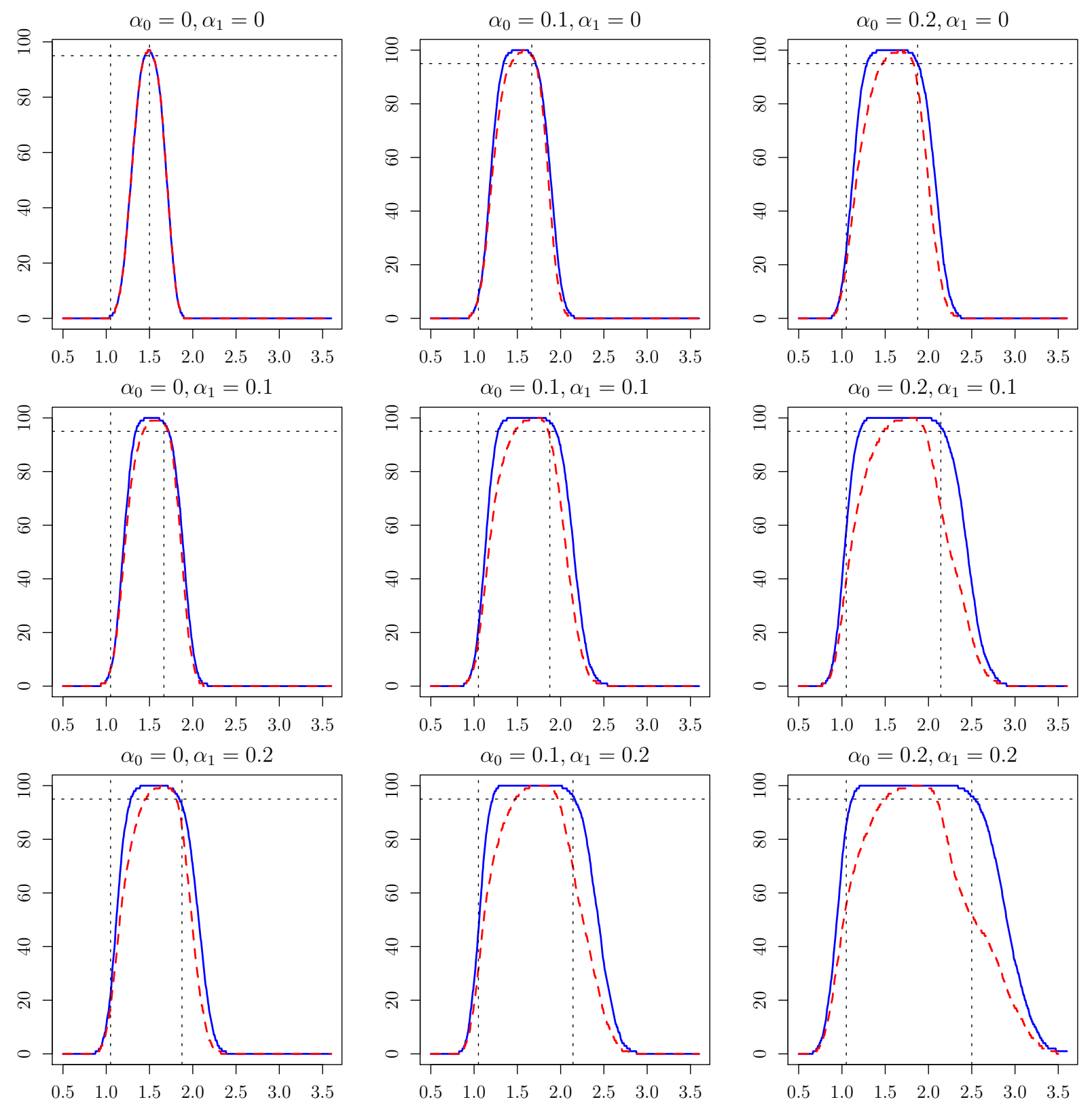

Figure D.21: Coverage Curves for Bonferroni versus Hybrid CIs: $\beta=1.5, n=1000$ 

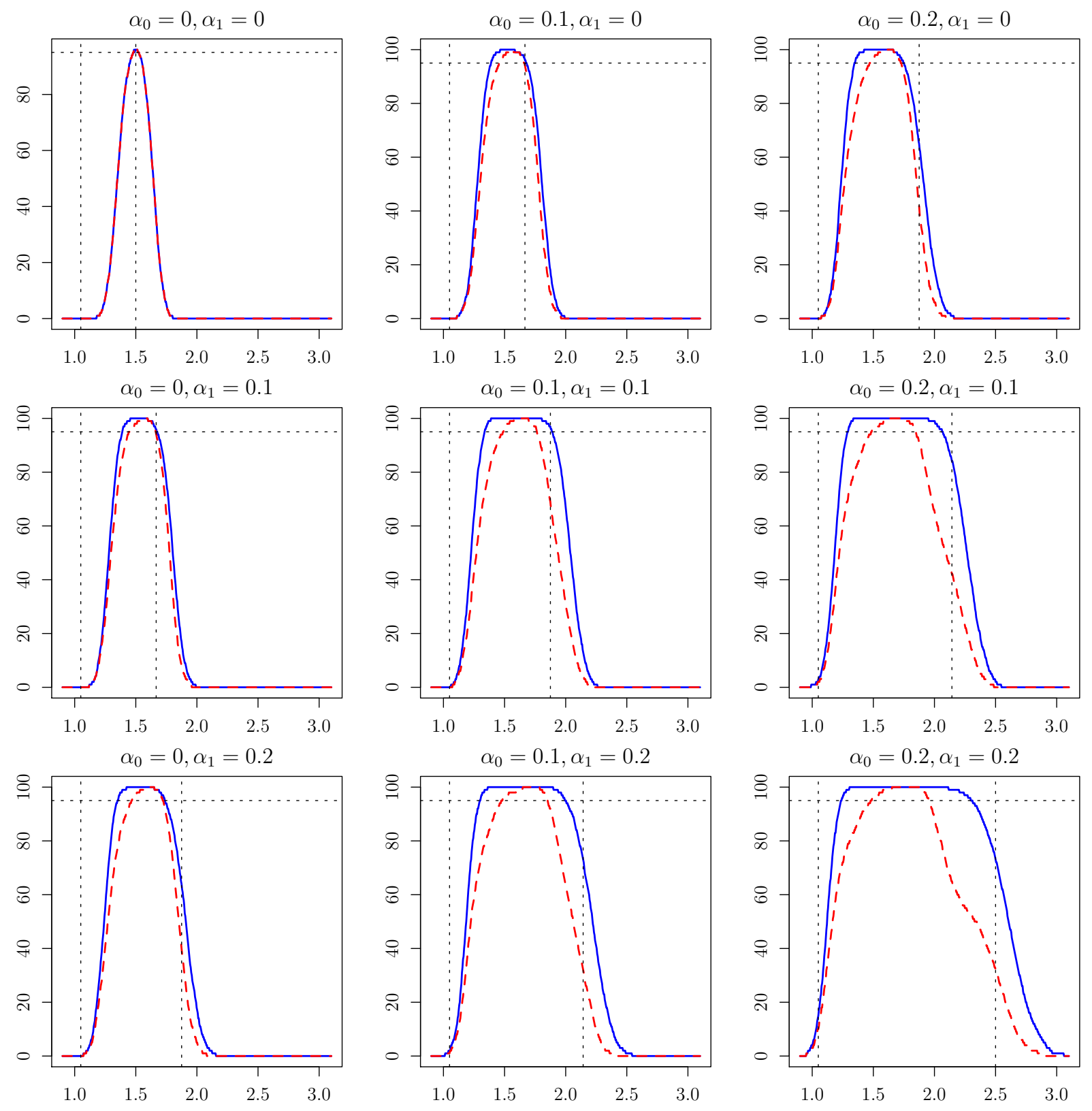

Figure D.22: Coverage Curves for Bonferroni versus Hybrid CIs: $\beta=1.5, n=2000$ 

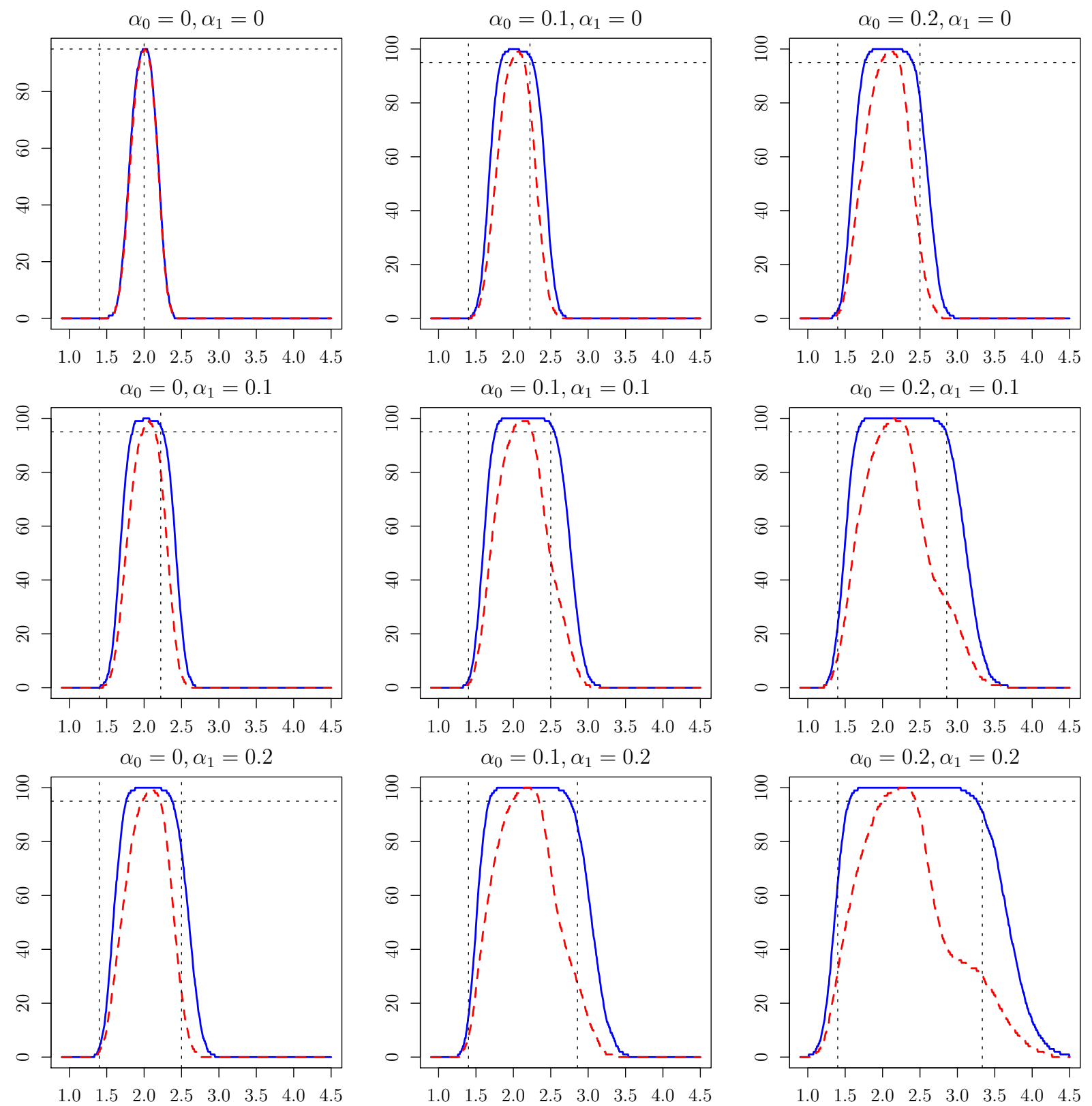

Figure D.23: Coverage Curves for Bonferroni versus Hybrid CIs: $\beta=2, n=1000$ 

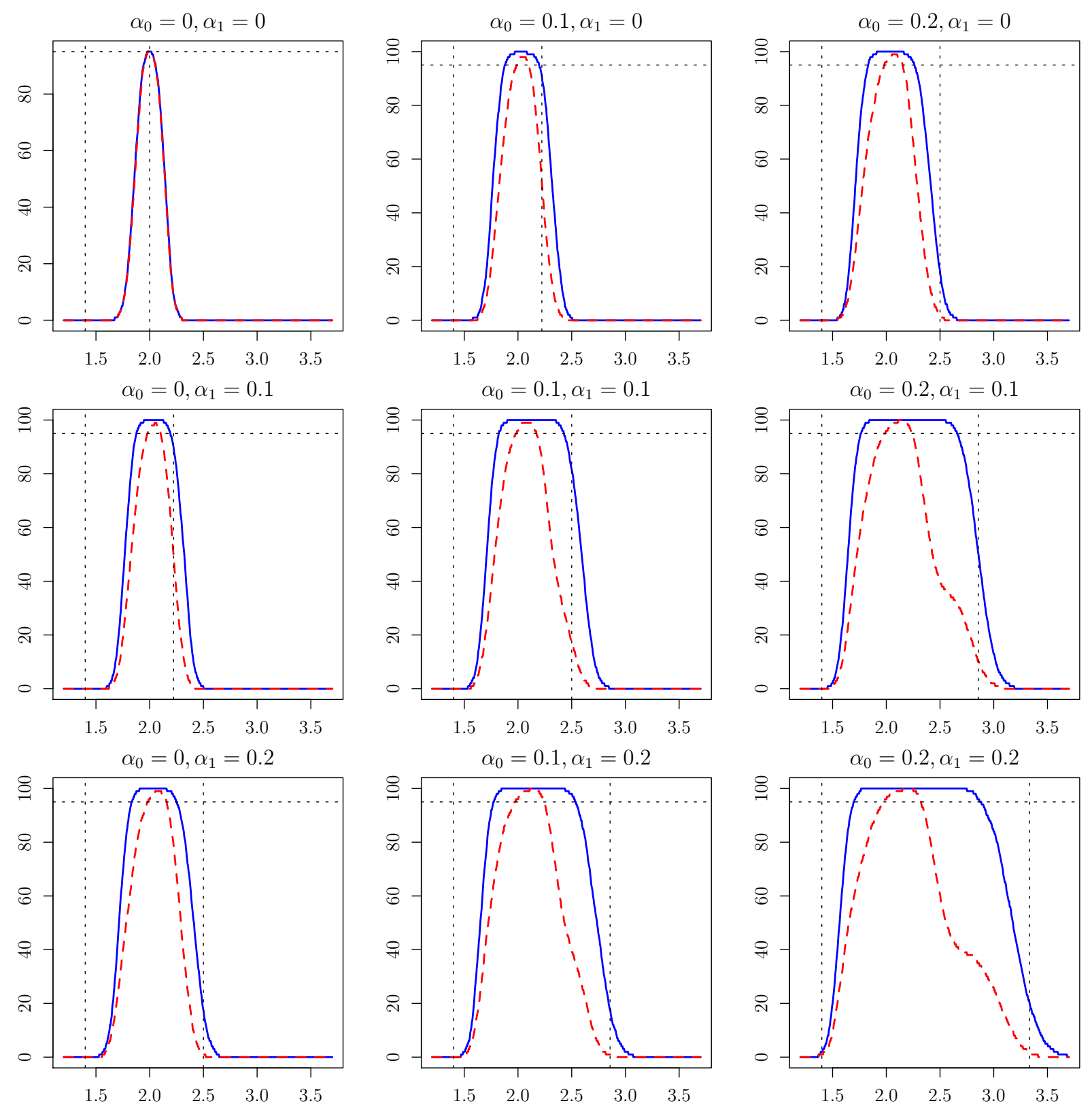

Figure D.24: Coverage Curves for Bonferroni versus Hybrid CIs: $\beta=2, n=2000$ 

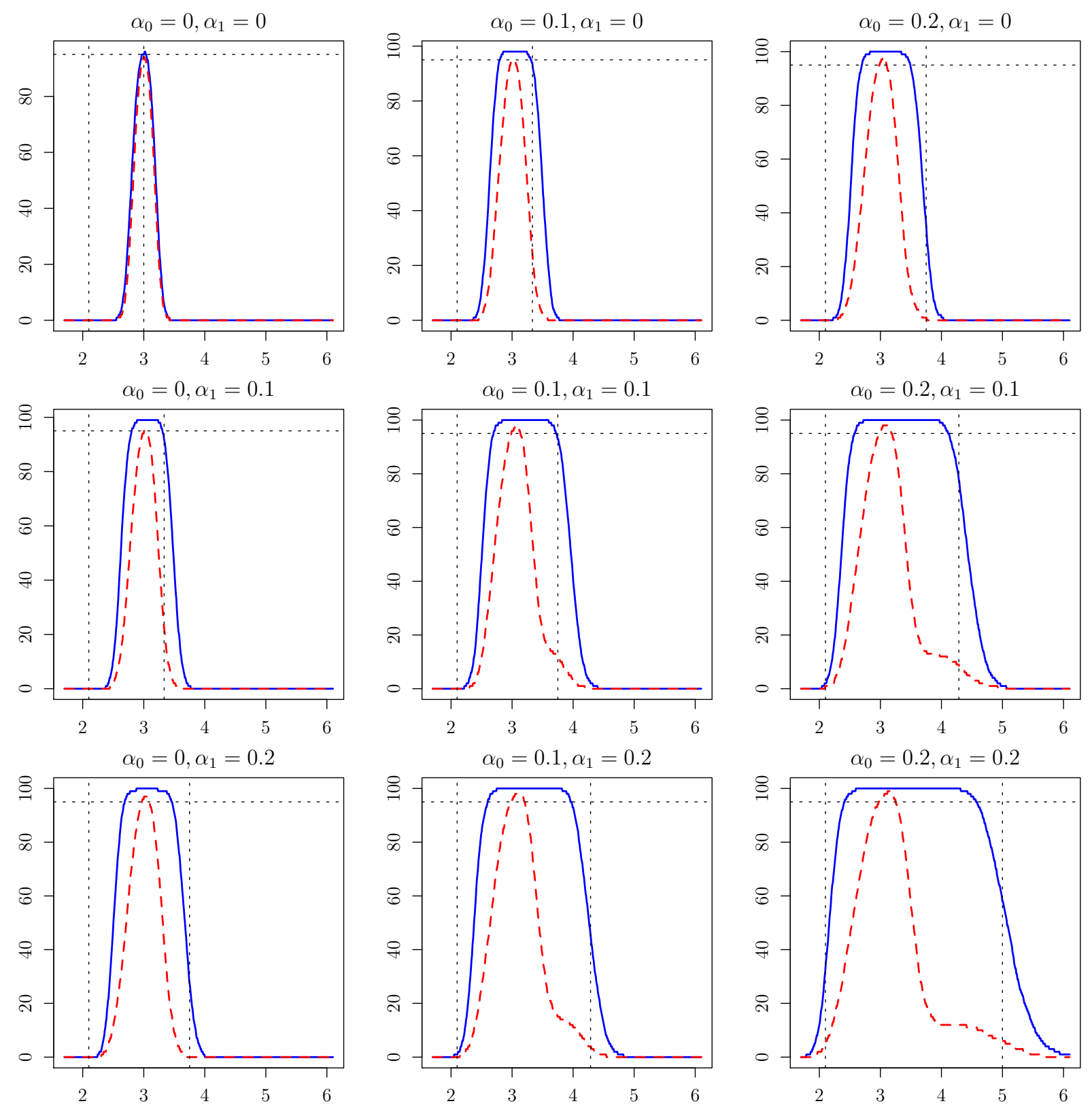

Figure D.25: Coverage Curves for Bonferroni versus Hybrid CIs: $\beta=3, n=1000$ 

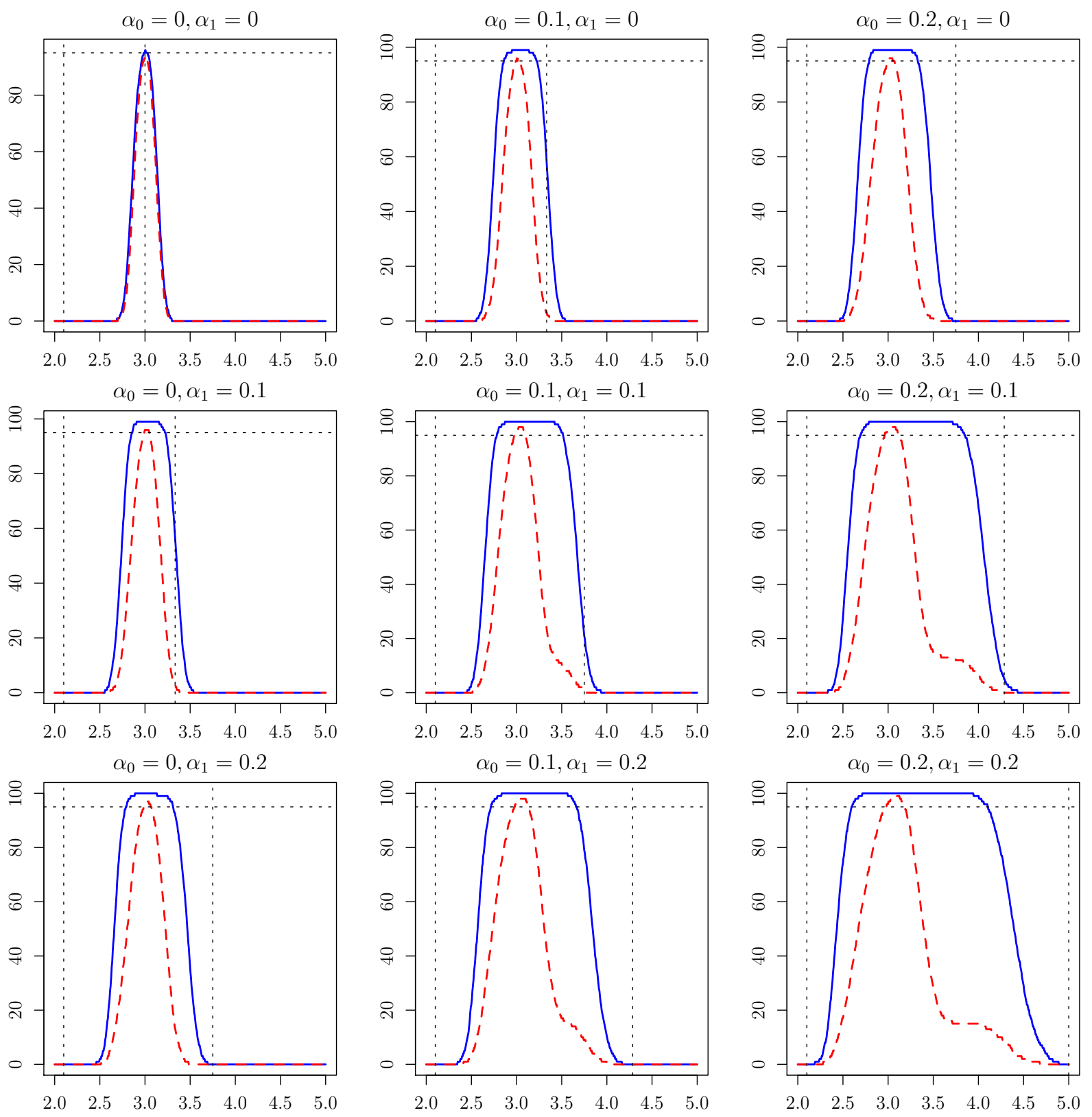

Figure D.26: Coverage Curves for Bonferroni versus Hybrid CIs: $\beta=3, n=2000$ 


\begin{tabular}{rr|rrrrrrrr}
\hline \hline & & \multicolumn{10}{|c}{$\beta$} \\
$\alpha_{0}$ & $\alpha_{1}$ & 0 & 0.25 & 0.5 & 0.75 & 1 & 1.5 & 2 & 3 \\
\hline 0.0 & 0.0 & 0.29 & 0.3 & 0.31 & 0.31 & 0.31 & 0.3 & 0.29 & 0.25 \\
& 0.1 & 0.32 & 0.35 & 0.4 & 0.44 & 0.48 & 0.48 & 0.36 & 0.33 \\
& 0.2 & 0.36 & 0.41 & 0.51 & 0.59 & 0.65 & 0.57 & 0.46 & 0.41 \\
& 0.3 & 0.41 & 0.48 & 0.64 & 0.76 & 0.79 & 0.68 & 0.56 & 0.5 \\
\hline 0.1 & 0.0 & 0.32 & 0.35 & 0.4 & 0.44 & 0.48 & 0.48 & 0.37 & 0.33 \\
& 0.1 & 0.36 & 0.41 & 0.51 & 0.6 & 0.68 & 0.65 & 0.48 & 0.43 \\
& 0.2 & 0.41 & 0.48 & 0.64 & 0.78 & 0.89 & 0.83 & 0.61 & 0.54 \\
& 0.3 & 0.48 & 0.59 & 0.82 & 1.02 & 1.09 & 0.98 & 0.75 & 0.65 \\
\hline 0.2 & 0.0 & 0.36 & 0.41 & 0.51 & 0.59 & 0.65 & 0.58 & 0.46 & 0.41 \\
& 0.1 & 0.41 & 0.48 & 0.65 & 0.79 & 0.9 & 0.89 & 0.61 & 0.54 \\
& 0.2 & 0.48 & 0.59 & 0.83 & 1.05 & 1.2 & 1.22 & 0.77 & 0.67 \\
& 0.3 & 0.57 & 0.73 & 1.09 & 1.4 & 1.58 & 1.53 & 0.97 & 0.85 \\
\hline 0.3 & 0.0 & 0.41 & 0.48 & 0.64 & 0.77 & 0.8 & 0.69 & 0.56 & 0.5 \\
& 0.1 & 0.48 & 0.59 & 0.83 & 1.02 & 1.13 & 1.19 & 0.75 & 0.65 \\
& 0.2 & 0.57 & 0.73 & 1.1 & 1.4 & 1.62 & 1.79 & 0.97 & 0.85 \\
& 0.3 & 0.72 & 0.95 & 1.49 & 1.93 & 2.36 & 1.58 & 1.25 & 1.1 \\
\hline
\end{tabular}

Table D.20: Median width of hybrid CI constructed from nominal 95\% GMM and nominal > 95\% Bonferroni intervals: $n=2000$ 